Article

\title{
Ultrahigh-Temperature Sphalerite from Zn-Cd-Se-Rich Combustion Metamorphic Marbles, Daba Complex, Central Jordan: Paragenesis, Chemistry, and Structure
}

\author{
Ella V. Sokol ${ }^{1}$, Svetlana N. Kokh ${ }^{1}$, Yurii V. Seryotkin ${ }^{1,2}{ }^{\circledR}$, Anna S. Deviatiiarova ${ }^{1, *}$, \\ Sergey V. Goryainov ${ }^{1}$, Victor V. Sharygin ${ }^{1,2}{ }^{-}$, Hani N. Khoury ${ }^{3}{ }^{\circledR}$, Nikolay S. Karmanov ${ }^{1}$, \\ Victoria A. Danilovsky ${ }^{1}$ and Dmitry A. Artemyev ${ }^{4,5}$ \\ 1 V.S. Sobolev Institute of Geology and Mineralogy, Siberian Branch of the Russian Academy of Sciences, \\ 3 Koptyug Avenue, Novosibirsk 630090, Russia; sokol@igm.nsc.ru (E.V.S.); s.n.kokh@gmail.com (S.N.K.); \\ yuvs@igm.nsc.ru (Y.V.S.); svg@igm.nsc.ru (S.V.G.); sharygin@igm.nsc.ru (V.V.S.); krm@igm.nsc.ru (N.S.K.); \\ victoria.saratovkina@gmail.com (V.A.D.) \\ 2 Department of Geology and Geophysics, Novosibirsk State University, Novosibirsk, 2 Pirogov Street, \\ Novosibirsk 630090, Russia \\ 3 Department of Geology, the University of Jordan, Amman 11942, Jordan; khouryhn@yahoo.com \\ 4 South Urals Federal Research Center of Mineralogy and Geoecology of the Urals Branch of the Russian \\ Academy of Sciences, Ilmeny Reserve, Miass 456317, Russia; daartemyev@gmail.com \\ 5 Geological Department, South Ural State University, Lenin av., 76, Chelyabinsk 454080, Russia \\ * Correspondence: anna13502@gmail.com or devyatiyarova@igm.nsc.ru; Tel.: +7-383-330-2149
}

Received: 11 August 2020; Accepted: 14 September 2020; Published: 17 September 2020

\begin{abstract}
Minerals of the Zn-Cd-S-Se system that formed by moderately reduced $\sim 800-850{ }^{\circ} \mathrm{C}$ combustion metamorphic (CM) alteration of marly sediments were found in marbles from central Jordan. Their precursor sediments contain Se- and Ni-enriched authigenic pyrite and ZnS modifications with high $\mathrm{Cd}$ enrichment (up to $\sim 10 \mathrm{wt} \%$ ) and elevated concentrations of $\mathrm{Cu}$, $\mathrm{Sb}, \mathrm{Ag}, \mathrm{Mo}$, and $\mathrm{Pb}$. The marbles are composed of calcite, carbonate-fluorapatite, spurrite, and brownmillerite and characterized by high $\mathrm{P}, \mathrm{Zn}, \mathrm{Cd}, \mathrm{U}$, and elevated $\mathrm{Se}, \mathrm{Ni}, \mathrm{V}$, and Mo contents. Main accessories are either $\mathrm{Zn}$-bearing oxides or sphalerite, greenockite, and $\mathrm{Ca}-\mathrm{Fe}-\mathrm{Ni}-\mathrm{Cu}$-O-S-Se oxychalcogenides. CM alteration lead to compositional homogenization of metamorphic sphalerite, for which trace-element suites become less diverse than in the authigenic ZnS. The CM sphalerites contain up to $\sim 14 \mathrm{wt} \% \mathrm{Cd}$ and $\sim 6.7 \mathrm{wt} \%$ Se but are poor in Fe (means 1.4-2.2 $\mathrm{wt} \%$ ), and bear 100-250 ppm Co, Ni, and Hg. Sphalerite $(\mathrm{Zn}, \mathrm{Cd}, \mathrm{Fe})(\mathrm{S}, \mathrm{O}, \mathrm{Se})_{\mathrm{cub}}$ is a homogeneous solid solution with a unit cell smaller than in $\mathrm{ZnS}_{\text {cub }}$ as a result of $\mathrm{S}^{2-} \rightarrow \mathrm{O}^{2-}$ substitution $(a=5.40852(12) \AA$, $V=158.211(6) \AA^{3}$ ). The amount of lattice-bound oxygen in the CM sphalerite is within the range for synthetic $\mathrm{ZnS}_{1-\mathrm{x}} \mathrm{O}_{\mathrm{x}}$ crystals $(0<x \leq 0.05)$ growing at $900{ }^{\circ} \mathrm{C}$.
\end{abstract}

Keywords: ZnS; sphalerite; würtzite; greenockite; trace elements; Mottled Zone; combustion metamorphism

\section{Introduction}

Various aspects of sphalerite geochemistry have received much attention in the literature ([1] and references therein). Most publications deal with sphalerite from economic $\mathrm{Zn}$ and $\mathrm{Pb}-\mathrm{Zn}$ deposits. The mineralogy of sulfides (including ZnS modifications) is often of little interest for metamorphic petrologists because sulfides reequilibrate much more quickly than silicate minerals [2] and thus do not represent the peak metamorphic conditions. However, sulfide assemblages were recently reported [3] to store records of changes in metamorphic environments and regimes. Brown et al. [3] considered 
several isochemical changes of sulfides for the case of blueschist and eclogite metamorphic rocks. They included (i) polymorphic transition of a phase, with no reactions between phases; (ii) composition changes of solid solutions in response to $P$-T changes; (iii) homogenization of a multiphase assemblage to a single phase (complex solid solution) upon heating; and (iv) breakdown of a complex phase to a multiphase assemblage of the same bulk composition upon cooling. The compositions of sulfides may also be interesting in terms of impurities and new mineral species: $P$ - $T$ changes and mineral growth in prograde reactions may be responsible for extremely high contents of impurities and rare minerals in high-grade metamorphic assemblages. Under these conditions, sulfides, unlike other rock-forming and accessory minerals, can accommodate chalcophile minor $(\mathrm{Cu}, \mathrm{Ni}, \mathrm{Co}, \mathrm{Zn}, \mathrm{As})$ and trace $(\mathrm{Cd}, \mathrm{Hg}$, $\mathrm{Tl}, \mathrm{Sb}, \mathrm{Se}, \mathrm{Te}, \mathrm{Pb}, \mathrm{Mo}, \mathrm{Bi}, \mathrm{Ag}$, Re) elements. This effect is especially impressive in Fe-poor high-grade marbles with rock-forming calcite and $\mathrm{Ca}(\mathrm{Mg})$ silicates [4-16]. They are specifically calcareous rocks produced by high-temperature low-pressure combustion metamorphism (CM) of marine chalky-marly sediments with variable percentages of phosphorite and clayey components and different abundances of minor and trace elements (P, S, Se, F, Sr, Ba, V, Cr, Ni, Cu, Ag, Zn, U) [4,14,17-22]. The CM marbles of central Jordan were derived mainly from the Belqa Group Maastrichtian-Early Eocene marly chalks. These bioproductive sediments have very high contents of redox-sensitive elements (mainly $\mathrm{Zn}, \mathrm{Cd}$, $\mathrm{U}$, as well as $\mathrm{Ni}, \mathrm{V}, \mathrm{Mo}, \mathrm{Cu}$, and $\mathrm{Se}$ ) and store disseminated organic matter (OM) with 9-11 wt\% of sulfidic sulfur $[4,14]$. The marbles formed by spontaneous ignition and combustion of OM and by annealing of the Belqa Gr. sediments at $700-850{ }^{\circ} \mathrm{C}$ at a shallow depth.

The CM rocks of the area have uniform rock-forming mineralogy with predominant calcite, less abundant spurrite and apatite supergroup minerals, minor brownmillerite, and scarce mayenite supergroup minerals. The marbles are remarkable for unusual and abundant accessory mineralization, with numerous $\mathrm{Zn}$ minerals. They contain primary sphalerite, zincite, tululite, and $\mathrm{Zn-rich}$ periclase and secondary qatranaite $\left(\mathrm{CaZn}_{2}(\mathrm{OH})_{6} \cdot 2 \mathrm{H}_{2} \mathrm{O}\right)$; diverse $\mathrm{Cd}$ compounds; various Ca uranates and their hydrated derivates; as well as Se-rich $\mathrm{Ni}$ and $\mathrm{Ag}$ chalcogenides. Zinc was revealed as an important impurity in primary brownmillerite, periclase, $(\mathrm{Ca}, \mathrm{Cd}) \mathrm{O}$, mayenite and spinel supergroup minerals, and in secondary Ca silicate hydrates (CSHs) [4,14,16,18-20,22-24].

This study focuses on high-temperature $\left(800-850{ }^{\circ} \mathrm{C}\right)$ spurrite-fluorapatite CM marbles from a site in central Jordan locally known as Tulul al Hammam. The rocks bear unique Zn mineralization, with $\mathrm{Zn}$ - and/or Cd-bearing oxides, abundant sphalerite, and sporadic greenockite crystals. Speculation about the maximal storage capacity of the sphalerite structure relative to a set of chalcophile elements is mainly based on the respective high-temperature $\left(\mathrm{T} \geq 700^{\circ} \mathrm{C}\right)$ phase diagrams, but too little is known about the real trace element composition of high- and ultra-high-temperature natural sphalerites because of their extremal rarity. The purpose of the current contribution was to provide for the first time analytical data characterizing the main and trace elements' composition as well as the structure of sphalerites from the Tulul al Hammam CM marbles with high Cd, Se, V, Cr, Ni, Cu, Ag, Zn, and U enrichment $[4,14,17-22]$. The goals of the study were (i) to obtain first constraints for $\left(\mathrm{Zn}_{1-\mathrm{x}} \mathrm{Me}_{\mathrm{x}}\right)\left(\mathrm{S}_{1-\mathrm{y}} \mathrm{A}_{\mathrm{y}}\right)$ complex solid solutions that formed in natural high-temperature and ambient-pressure environments; (ii) to reveal relationships in trace-element (TE) compositions of CM sphalerite and the primary TE hosts from precursor sediments; (iii) to constrain trends of TE fractionation into CM sphalerite; and (iv) to understand the reasons for minor- and trace-element substitutions in $\mathrm{ZnS}_{\text {cub }}$ during natural high-temperature low-pressure alteration.

A combination of several advanced analytical techniques was applied to better resolve lattice-hosted elements and those in nano- and microinclusions [1], epitaxial multi-phase 'sandwiches' $\left(\mathrm{ZnS}_{\mathrm{cub}}, \mathrm{ZnS}_{\mathrm{hex}}, \mathrm{CdS}, \mathrm{ZnO}\right)$ [25-29], and in other defects, which may be problematic to discriminate even at the scale of an electron microprobe beam. CM sphalerite crystals were characterized by electron probe microanalyses (EPMA), scanning electron microscopy with energy dispersive spectroscopy (SEM-EDS), elemental mapping, laser-ablation inductively coupled plasma mass spectrometry (LA-ICPMS), single-crystal X-ray diffractometry (XRD), and single-crystal Raman spectroscopy. 


\section{Geological Background}

The CM rocks of the Daba-Siwaqa complex (Mottled Zone, central Jordan) known under a local name of varicolored marbles bear diverse $\mathrm{Zn}$ mineralization together with various $\mathrm{Ca}-\mathrm{U}(\mathrm{VI})$ oxides and Cd-rich compounds [18-20,22]. The rocks were derived from marine sediments of the Belqa Group cropping out ubiquitously in the area (Figures 1 and 2). The sediments were deposited at shallow sea depths in a stable highly productive shelf environment between the Late Cretaceous and the Early Eocene (ca. 90-50 Ma ago) [4,14,18,30,31]. Their gentle folding and faulting were mostly related to continued tectonic movements along the Dead Sea-Jordanian Transform located $\sim 60 \mathrm{~km}$ to the west of Daba-Siwaqa [31]. The organic-rich sediments were uplifted in the Quaternary and became exposed to spontaneous oxidation and in situ combustion of the hosted bitumen and disseminated sulfides within the tectonically disturbed zones. For details of the local geology, see [17,18,32].

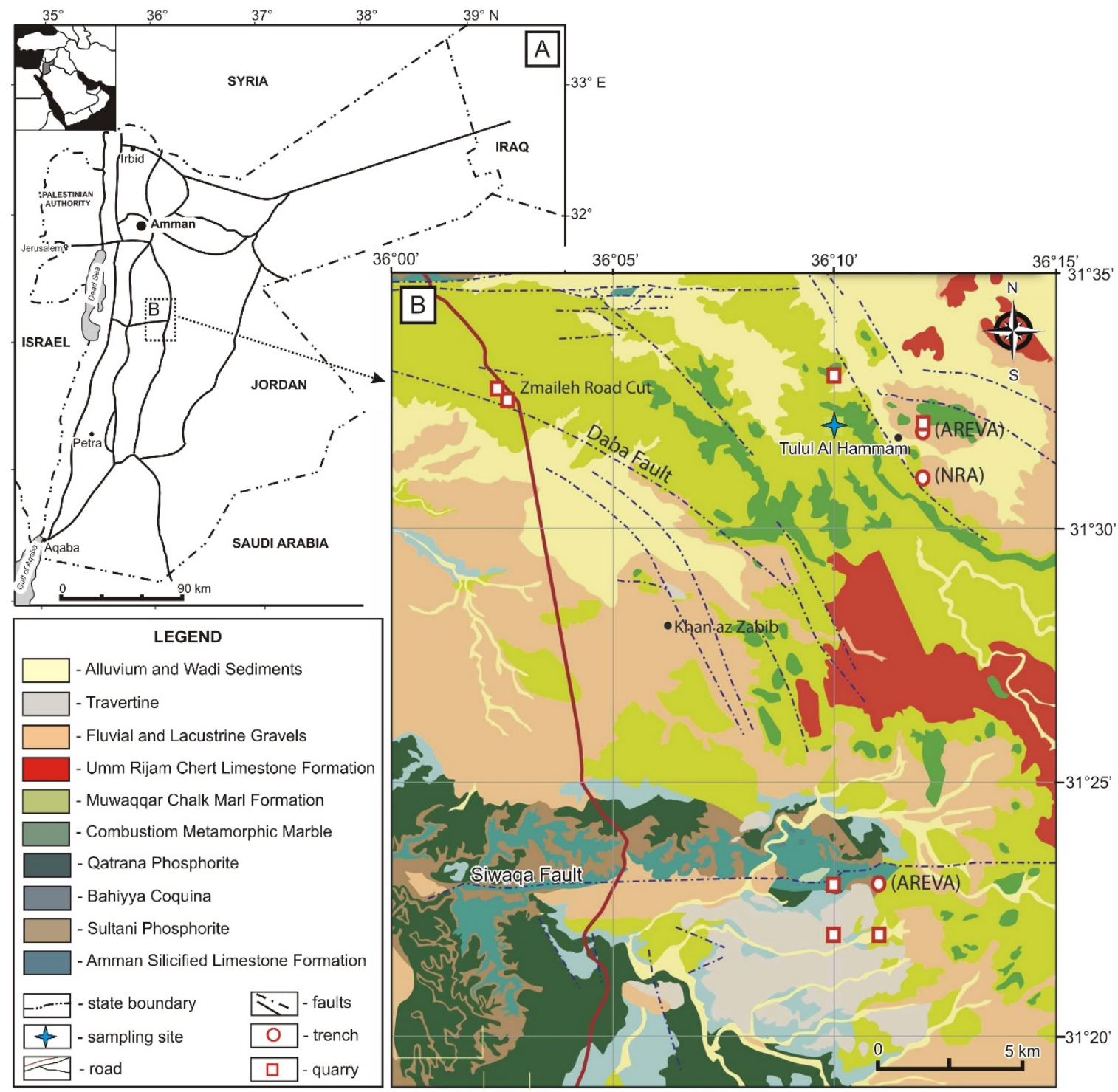

Figure 1. (A) Location map of the Daba-Siwaqa complex, modified after [14]. (B) Detailed geological map of the Daba-Siwaqa complex showing the location of the Tulul al Hammam area, modified after [18].

In many outcrops, marbles are rather fresh and preserve the original lamination of the precursor sediments (Figure 3A). Fresh marbles rich in disseminated sulfides are black, while the altered varieties, 
as well as those enriched in fresh brownmillerite and/or Ca-ferrites, are brown; strongly altered marbles are pale red or pink. Green marbles are rich in fluorapatite and/or fluorellestadite (Figure 3C,D). Fresh samples often show thin $(\leq 3 \mathrm{~cm})$ bands of contrasting colors that reflect an uneven distribution of sulfur-rich organic matter ( \pm sulfides) and phosphates in the protoliths.

The type locality of CM sphalerite called Tulul al Hammam (Arabic for Pigeon's Hill) (31 $32^{\prime} \mathrm{N}$ $36^{\circ} 12^{\prime} \mathrm{E}$ ) is situated in the northern Siwaqa area (Figure 1B) $[18,32]$. Varicolored CM marbles typically occur as lenticular bodies from a few meters to more than $60 \mathrm{~m}$ thick and build cliffs or hills. The marbles are derived from the Maastrichtian-Paleogene Muwaqqar Fm. chalk and marl enriched in phosphorous, organic matter, and trace elements, including $\mathrm{Cd}, \mathrm{Zn}$, and $\mathrm{U}$. The whole sequence crops out along valleys (wadis), where bituminous chalk and marl lie under CM rocks, unconformable Pleistocene travertine, fluvial to lacustrine deposits, and Holocene to Present alluvium.

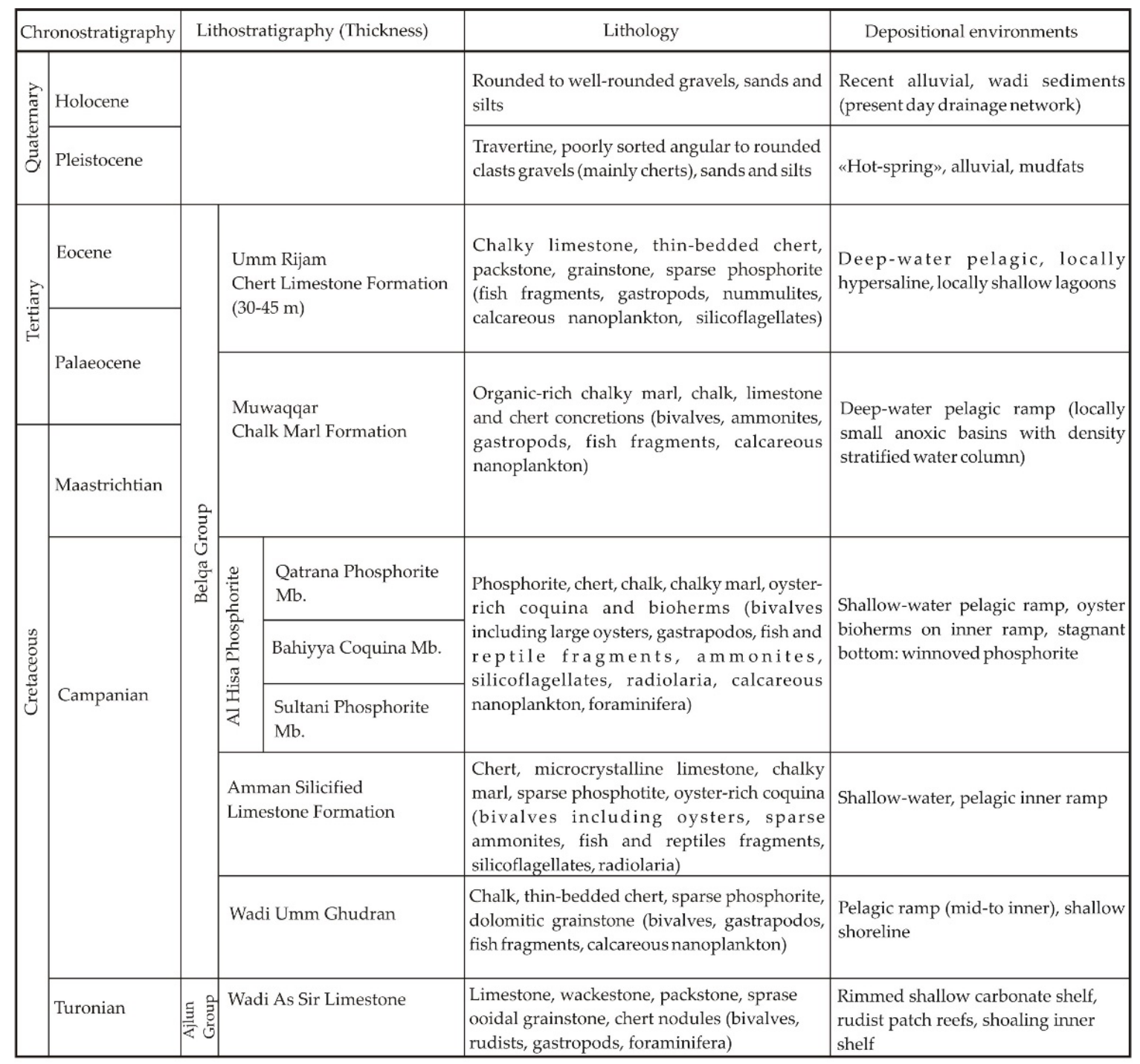

Figure 2. Synthesised stratigraphic nomenclature: lithostratigraphy and lithology of central Jordan. Compiled data from $[4,31]$. Mb. = Member. 

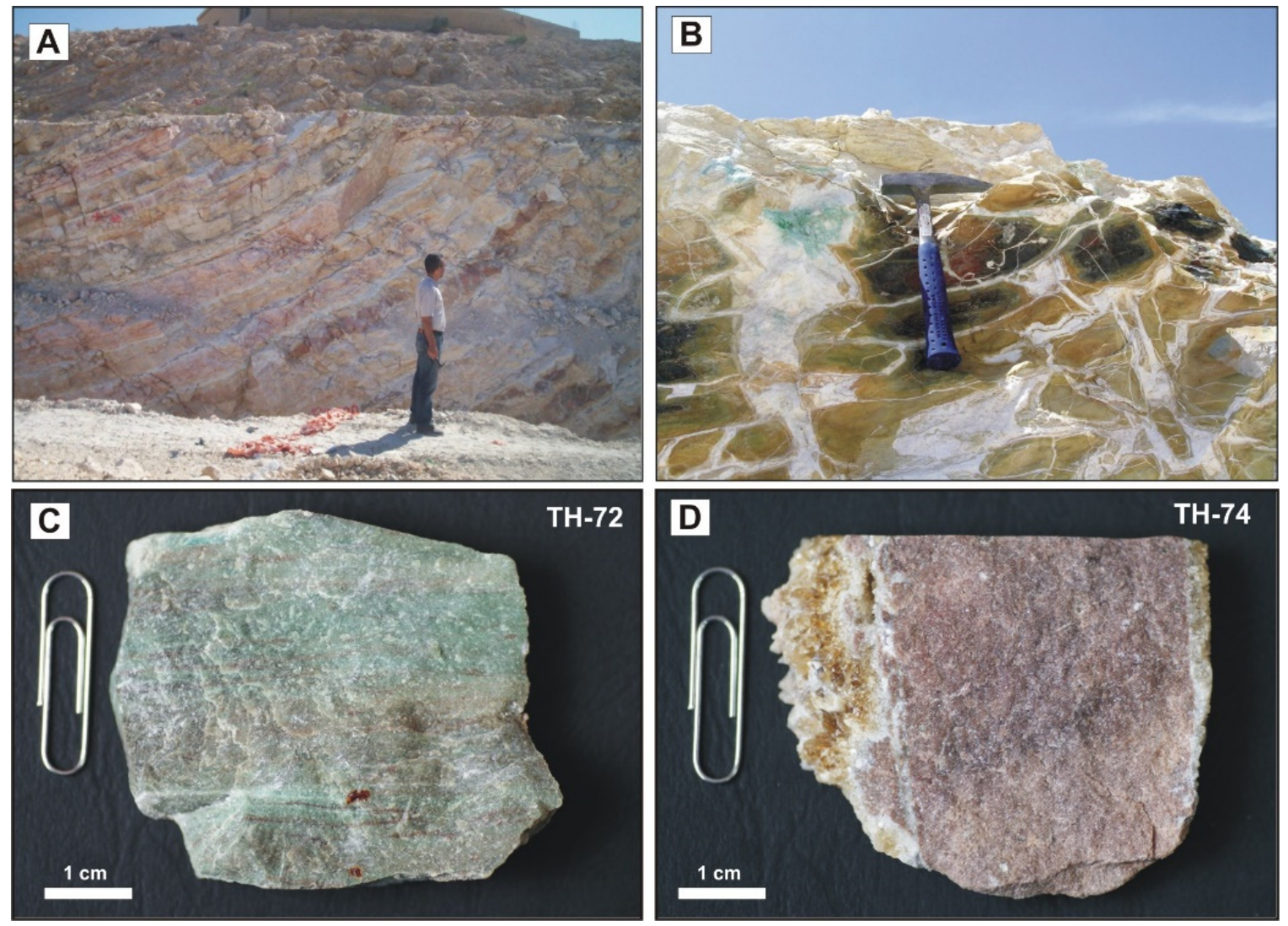

Figure 3. The varicolored marbles from Daba-Siwaqa complex (central Jordan). (A) Exposures of varicolored marbles with the original textural features of precursory sediments, after [18]. (B) Varicolored marbles with strong retrograde alteration. (C) Sample of spurrite-fluorapatite marble with abundant primary Zn-bearing oxide compounds. (D) Sample of ZnS-bearing fluorapatite marble.

\section{Materials and Methods}

The initial scrutiny of about $60 \mathrm{Zn}$-enriched marble samples from the Tulul al Hammam quarries showed that approximately one sample per 10 contains different percentages of sphalerite and/or $(\mathrm{Cd}, \mathrm{Zn})(\mathrm{S}, \mathrm{Se})$ solid solutions, besides predominant oxygen-bearing primary and secondary $\mathrm{Zn}$ minerals. Seven samples of ZnS-bearing marbles, with 230 to $1440 \mathrm{ppm} \mathrm{Zn,} \mathrm{Zn/Cd} \mathrm{from} 4.4$ to 77, and 4 to 500 ppm Se (DT-20, DT-25, DT-26, TH-11, TH-52, TH-74, and TH-79) were selected for detailed analytical work (Table 1). The analyses were carried out mainly at the Analytical Center for Multi-Elemental and Isotope Research (Sobolev Institute of Geology and Mineralogy (IGM), Novosibirsk, Russia) and at the South Urals Federal Research Center of Mineralogy and Geoecology (SU FRC MG) (Miass, Russia).

Major elements in bulk samples of marbles and chalky sediments were analyzed by the solution ICP-OES technique on a Thermo Jarrell Intertechs IRIS Advantage atomic emission spectrometer (USA) at IGM (Novosibirsk). The preconditioning procedure included fusion of powdered whole rock samples with lithium borate as in [33].

Trace elements in bulk samples of marbles and chalky sediments were determined by ICP-MS on an Agilent 7700x spectrometer (USA) at the SU FRC MG (Miass, Russia). For this, 50-mg specimens were digested in a mixture of $2 \mathrm{~mL} 69 \% \mathrm{HNO}_{3}, 6 \mathrm{~mL} \mathrm{30 \%} \mathrm{HCl}$, and $2 \mathrm{~mL}$ of $40 \% \mathrm{HF}$ in closed Teflon bombs using a Berghof SpeedWave microwave digestion system. The procedure was run in steps, programmed to a temperature of $180{ }^{\circ} \mathrm{C}$ and a ramp time of $5 \mathrm{~min}$ at step 1 and $180{ }^{\circ} \mathrm{C}, 5 \mathrm{~min}$ hold time, a constant power of $500 \mathrm{~W}$, and a pressure of 20 bar at step 2 . Then, the residue was re-dissolved in $5 \mathrm{~mL} \mathrm{HCl}(1: 1 \mathrm{~V} / \mathrm{V})$ and evaporated. Finally, the residue was dissolved in $10 \mathrm{~mL}$ of $\mathrm{HNO}_{3}(20 \%$; $1: 5$ $V / V)$ and the solution was heated at $150{ }^{\circ} \mathrm{C}$ for $30 \mathrm{~min}$. 
Table 1. Trace-element compositions of sphalerite-bearing CM marbles, Tulul al Hammam area (Daba-Siwaqa complex), and their sedimentary protoliths ('oil shales') of the Muwaqqar Chalk Marl Formation (ppm).

\begin{tabular}{|c|c|c|c|c|c|c|c|c|c|c|c|c|}
\hline \multirow[b]{2}{*}{ Element } & \multicolumn{8}{|c|}{ Spurrite-Fluorapatite Marbles } & \multicolumn{4}{|c|}{ Muwaqqar Fm. 'Oil Shales' } \\
\hline & DT-20 & DT-26 & DT-25 & TH-11 & TH-74 & TH-52 & TH-79 & TH-72* & JRD10-12E ** & DOS-1 & OSB & JRD10-14 ** \\
\hline $\mathrm{V}$ & 321 & 186 & 1152 & 130 & 52.0 & 235 & 55.0 & 457 & 499 & 357 & 186 & 338 \\
\hline $\mathrm{Cr}$ & 422 & 984 & 1880 & 119 & 36.3 & 71.0 & 98.0 & 579 & 266 & 412 & 356 & 452 \\
\hline $\mathrm{Mn}$ & 22.9 & 40.0 & 40.7 & 30.0 & 80.0 & 30.0 & 80.0 & 30.0 & na & 19.5 & 32.0 & na \\
\hline Co & 1.65 & na & 2.16 & na & na & na & na & na & 9.68 & 2.44 & 4.10 & 5.12 \\
\hline $\mathrm{Ni}$ & 273 & 290 & 684 & 119 & 115 & 103 & 158 & 457 & 335 & 256 & 93.0 & 182 \\
\hline $\mathrm{Cu}$ & 164 & 126 & 329 & 28.1 & 18.8 & 25.6 & 36.8 & 220 & 50.0 & 104 & 67.0 & 77.9 \\
\hline $\mathrm{Zn}$ & 1519 & 1440 & 1091 & 470 & 333 & 329 & 231 & 1437 & 2321 & 1498 & 632 & 428 \\
\hline $\mathrm{Ga}$ & 2.17 & 3.89 & 7.20 & 1.50 & 2.80 & 1.49 & 2.32 & 2.80 & na & 8.78 & 8.60 & na \\
\hline $\mathrm{Ge}$ & na & $<0.20$ & na & $<0.20$ & 0.35 & 0.34 & 0.60 & 0.22 & na & 0.55 & $<0.20$ & na \\
\hline As & 24.2 & 30.5 & 34.4 & 11.9 & 81.9 & 23.9 & 14.9 & $<6.0$ & 145 & 16.0 & 21.1 & 22.0 \\
\hline $\mathrm{Se}$ & 38.4 & 181 & 503 & 6.50 & 4.00 & 6.31 & 13.9 & 595 & na & 94.9 & 32.5 & na \\
\hline $\mathrm{Sr}$ & 1271 & 1360 & 1349 & 968 & 967 & 1044 & 1565 & 1590 & 462 & 603 & 683 & 692 \\
\hline Y & 41.8 & 33.0 & 46.4 & 25.8 & 13.0 & 8.60 & 8.30 & 26.1 & na & 27.6 & 13.2 & na \\
\hline $\mathrm{Zr}$ & 19.3 & 59.0 & 35.3 & 21.2 & 20.7 & 21.2 & 33.6 & 67.0 & na & 34.2 & 52.9 & na \\
\hline $\mathrm{Nb}$ & 0.80 & 1.88 & 0.80 & 0.90 & 0.90 & $<0.2$ & 0.80 & 0.90 & na & 0.73 & 5.70 & na \\
\hline Mo & 9.78 & 31.8 & 70.3 & 12.6 & 3.20 & 5.30 & 1.50 & 6.04 & 803 & 178 & 70.9 & 951 \\
\hline $\mathrm{Ag}$ & na & na & 8.10 & 4.60 & 42.6 & $<1.9$ & 5.40 & 16.9 & na & na & na & na \\
\hline $\mathrm{Cd}$ & 60.9 & 39.0 & 41.4 & 28.2 & 75.0 & 25.9 & 3.00 & 697 & 98.2 & 224 & 69.0 & 85.3 \\
\hline $\mathrm{Sb}$ & 5.11 & na & 12.5 & $<2.7$ & $<2.7$ & 4.40 & 4.20 & 11.2 & 18.74 & na & na & 6.93 \\
\hline $\mathrm{Ba}$ & 136 & 171 & 424 & 253 & 212 & 472 & 190 & 134 & 40.5 & 58.1 & 45.0 & 43.1 \\
\hline $\mathrm{Hg}$ & 0.007 & 0.003 & 0.003 & na & na & na & na & $<0.002$ & na & 0.014 & 0.025 & na \\
\hline $\mathrm{Pb}$ & 2.00 & 4.50 & 0.22 & 0.80 & 1.40 & 1.60 & 1.20 & 14.5 & 5.53 & 12.0 & 4.90 & 6.94 \\
\hline Th & 0.31 & 0.76 & 0.50 & 4.00 & 2.90 & 4.90 & 6.70 & 7.30 & 1.79 & 1.98 & 4.40 & 2.23 \\
\hline $\mathrm{U}$ & 24.0 & 24.0 & 15.1 & 9.10 & 6.20 & 3.90 & 3.90 & 24.0 & 134 & 36.6 & 14.1 & 455 \\
\hline $\mathrm{Zn} / \mathrm{Cd}$ & 24.9 & 34.8 & 26.4 & 16.7 & 4.4 & 12.7 & 77.0 & 2.06 & 23.6 & 6.7 & 9.2 & 5.0 \\
\hline
\end{tabular}


After cooling at room temperature, the solution was poured into $100-\mathrm{mL}$ volumetric flasks and then filled with ultrapure water till the final designed volume for analysis. The quality control and quality assurance procedures included processing and analysis, at each batch, of two blanks and the BCR-2 certified reference material (NIST, USA). Minor and trace elements were quantified using 5-point daily calibration. The analyses were run in triplicate, and the results differed for less than 5\%. Two blanks were prepared and included 10 samples in each batch. The procedural blanks accounted for less than $1 \%$ of the element concentrations. Precision and accuracy were estimated to be 10-15 rel\% for all elements. Method detection limits for trace elements were in the range 0.01-0.5 $\mu \mathrm{g} / \mathrm{L}$. The analytical reproducibility was monitored using the BCR-2 standard, in which the measured values were consistent with the certified ones, with extraction efficiencies ranging from $93 \%$ to $120 \%$. The method was slightly modified after those reported in [34,35].

Bulk $\mathrm{Hg}$ contents in the marble samples were measured by flameless atomic absorption spectrometry (AAS) on a Lumex RA-915M Hg analyzer (Lumex Ltd., Saint Petersburg, Russia) with an RP-91C pyrolysis attachment. Prior to analyses, the rock samples were powdered in a mortar and homogenized. The technical specifications of the instrument allow special preconditioning of solid samples to be avoided. The national standard of soil (SDPS-3) certified for heavy metals and mercury was used to calibrate the spectrometer and to check the quality of analyses. Relative analytical errors were $20 \%(p=0.95)$ for concentrations from $5 \times 10^{-7}$ to $2.5 \cdot \times 10^{-2} \mathrm{wt} \%$ [36].

Scanning electron microscopy (SEM) was applied to identify minerals and to characterize the phase distribution and morphology of Zn-bearing phases, based on energy-dispersive spectra (EDS), back-scattered electron (BSE) images, and elemental maps (EDS system). The measurements were performed at IGM (Novosibirsk) on a Tescan Orsay TESCAN MIRA 3LMU microscope (Tescan Orsay Holding, Brno, Czech Republic) equipped with an Oxford Instruments AZtec Energy XMax-50 microanalyses system (Oxford Instruments Nanoalysis, Abingdon, UK). Thin sections were repolished immediately before analyses, and fresh cleavage surfaces were sputter coated with $\sim 15-$ to $25-\mathrm{nm}$ carbon films for SEM examination. EDS analyses of minerals were run in a high-vacuum mode at an accelerating voltage of $20 \mathrm{kV}$, a beam current of $1.5 \mathrm{nA}$, and a live spectra acquisition time of $20 \mathrm{~s}$. The following synthetic compounds, minerals, and pure elements were used as reference samples for the elements analysed: $\mathrm{SiO}_{2}(\mathrm{Si}$ and $\mathrm{O}), \mathrm{Al}_{2} \mathrm{O}_{3}(\mathrm{Al})$, diopside $(\mathrm{Mg}$ and $\mathrm{Ca})$, albite $(\mathrm{Na})$, orthoclase $(\mathrm{K})$, $\mathrm{Ca}_{2} \mathrm{P}_{2} \mathrm{O}_{7}(\mathrm{P}), \mathrm{BaF}_{2}(\mathrm{Ba}$ and $\mathrm{F}), \mathrm{FeS}_{2}(\mathrm{~S}), \mathrm{Cr}_{2} \mathrm{O}_{3}(\mathrm{Cr}), \mathrm{CsRe}_{2} \mathrm{Cl}_{6}(\mathrm{Cl}), \mathrm{SrF}_{2}(\mathrm{Sr}), \mathrm{Ti}, \mathrm{Fe}, \mathrm{Mn}, \mathrm{Zn}, \mathrm{Ni}, \mathrm{Cu}, \mathrm{V}$, $\mathrm{Ag}, \mathrm{Cd}$, and Se.

Matrix correction was done using the XPP algorithm (exponential model of Pouchou and Pichoir matrix correcrion), implemented in the software of the microanalysis system. Metallic Co served for quantitative optimization (normalization to beam current and energy calibration of the spectrometer) [37-39]. The detection limits ( $3 \sigma$ value) were $0.10 w t \%$ for $\mathrm{K}, \mathrm{Ca}, \mathrm{Fe}$, and $\mathrm{Ni} ; 0.15 \mathrm{wt} \%$ for $\mathrm{Se}, \mathrm{Cu}, \mathrm{Ag}$, and $\mathrm{Cd}$; and $0.40 \mathrm{wt} \%$ for $\mathrm{O}$.

The variation coefficients that characterize the repeatability of a single determination were found to be $\sim 0.9 \mathrm{rel} \%$ for EDS in the compositional range of the main mineral-forming components $([\mathrm{C}]>10 \mathrm{wt} \%) ; 3 \mathrm{rel} \%$ for minor components $(1 \mathrm{wt} \%<[\mathrm{C}]<10 \mathrm{wt} \%)$, and $\sim 13 \mathrm{rel} \%$ for impurities $(0.3 \mathrm{wt} \%<[\mathrm{C}]<1 \mathrm{wt} \%)$ [37]. EDS was chosen as the principal method for fast identification of mineral phases and determination of their major- and trace-element compositions. Scanning electron microscopy in the BSE mode and elemental mapping were used to characterize various texture aspects in sphalerite-bearing mineral intergrowths, with a special focus on inhomogeneities in sphalerite grains (zoning and mineral inclusions).

Electron probe microanalyses (EPMA). Mineral chemistry of sulfides was analyzed in $>10-\mu \mathrm{m}$ grains on a Jeol JXA 8100 electron microprobe microanalyzer (Jeol, Tokio, Japan) in C-coated polished thin sections, at an accelerating voltage of $20 \mathrm{keV}$, a beam current of $20 \mathrm{nA}$, and a peak counting time of $10 \mathrm{~s}$. The compositions of sphalerite and würtzite were determined with reference to standards: synthetic $\mathrm{ZnS}$ for $\mathrm{S} \mathrm{K} \alpha$ and $\mathrm{Zn} \mathrm{K} \alpha, \mathrm{CuFeS}_{2}$ for Fe $\mathrm{K} \alpha$ and $\mathrm{Cu} \mathrm{K} \alpha, \mathrm{CdS}$ for $\mathrm{Cd} \mathrm{L} \alpha$, InAs for In $\mathrm{L} \alpha$ and $\mathrm{As} \mathrm{L} \alpha, \mathrm{ZnSe}$ for Se $\mathrm{L} \alpha, \mathrm{Sb}_{2} \mathrm{~S}_{3}$ for $\mathrm{Sb} \mathrm{L} \alpha$, FeNiCo for $\mathrm{Ni} \mathrm{K} \alpha$, and $\mathrm{PbTe}$ for Te $\mathrm{L} \alpha$. The detection 
limits ( $3 \sigma$ value) for the elements were $0.03 \mathrm{wt} \%$ for $\mathrm{Ni} ; 0.06 \mathrm{wt} \%$ for $\mathrm{S}$, $\mathrm{As}, \mathrm{In}, \mathrm{Cd}$, and $\mathrm{Sb} ; 0.09 \mathrm{wt} \%$ for $\mathrm{Cu}, \mathrm{Zn}$ and Te; and $0.2 \mathrm{wt} \%$ for Se. The matrix correction using the ZAF algorithm (generalized algebraic procedure; assumes a linear relation between concentarion and X-ray intensity) [40] was applied to raw data prior to recalculation into elements. Analytical accuracy was within $2 \mathrm{rel} \%$ for $[C]>5 \mathrm{wt} \%$ elements, and about $5 \mathrm{rel} \%$ for $[\mathrm{C}]<2 \mathrm{wt} \%$ elements.

LA-ICPMS. The trace-element chemistry of $\mathrm{Zn}$ and Fe sulfides was determined by laser ablation mass spectrometry with inductively coupled plasma (LA-ICPMS) at the SU FRC MG (Miass). Phalerite was analyzed in polished sections of three selected coarse-grained samples (marble samples DT-20 and DT-25), würtzite and pyrite (sediment sample DOS-1) preconditioned using the standard equipment, and following the standard protocol [41]. The texture of the samples was examined by SEM with a special focus on inhomogeneities in $\mathrm{ZnS}$ and $\mathrm{FeS}_{2}$ crystals (zoning or mineral inclusions). The selected large $(50-200 \mu \mathrm{m})$ sulfide crystals are chemically homogeneous and generally free from visible inclusions or signatures of alteration. The LA-ICPMS method was of limited applicability for sphalerites from other marble samples, because they were too small (within $20 \mu \mathrm{m}$ across) and often enclosed extraneous phases. The concentrations of $\mathrm{Zn}$ and Fe determined by EDS and WDS were used as internal standards for the LA-ICPMS trace- and minor-element data.

The LA-ICPMS analysis was run on a NewWave Research UP-213 laser ablation system coupled with an Agilent 7700x (Agilent Technologies, Inc., Santa Clara, CA, USA) plasma mass spectrometer. The procedure was as in [42], with an Nd: YAG UV source, frequency quadruple (wavelength $213 \mathrm{~nm}$ ) with fluence settings of $2.5-3.5 \mathrm{~J} / \mathrm{cm}^{2}$ for pyrite and 3.5-5.0 for sphalerite and würtzite, helium cell carrier gas, and a gas flow rate of $0.6-0.7 \mathrm{~L} / \mathrm{min}$. The mass spectrometer settings were as follows: $1550 \mathrm{~W}$ RF power; Ar as a carrier gas; a flow rate of 0.95 to $1.05 \mathrm{~L} / \mathrm{min}$; a plasma gas flow (Ar) of $15 \mathrm{~L} / \mathrm{min}$; and an auxiliary gas flow (Ar) of $0.9 \mathrm{~L} / \mathrm{min}$. Each analysis was performed with a laser spot size of 30-100 $\mu \mathrm{m}$ at a frequency of $10 \mathrm{~Hz}$. Each sample was measured for $90 \mathrm{~s}$ (30-s background measurements plus 60-s analysis), with 5-s pre-ablation before each analysis and 30-s washout between the analyses. The mass spectrometer was tuned with NIST SRM-612 glass. The Th/U ratio is $\sim 1$. Production of molecular oxide species (i.e., ${ }^{232} \mathrm{Th}^{16} \mathrm{O} /{ }^{232} \mathrm{Th}$ ) was maintained at $<0.2 \%$. The monitored isotopes included ${ }^{51} \mathrm{~V},{ }^{53} \mathrm{Cr},{ }^{55} \mathrm{Mn},{ }^{57} \mathrm{Fe},{ }^{59} \mathrm{Co},{ }^{60} \mathrm{Ni},{ }^{65} \mathrm{Cu},{ }^{66} \mathrm{Zn},{ }^{69} \mathrm{Ga},{ }^{72} \mathrm{Ge},{ }^{75} \mathrm{As},{ }^{77} \mathrm{Se},{ }^{95} \mathrm{Mo},{ }^{107} \mathrm{Ag}$, ${ }^{111} \mathrm{Cd},{ }^{115} \mathrm{In},{ }^{118} \mathrm{Sn},{ }^{121} \mathrm{Sb},{ }^{125} \mathrm{Te},{ }^{197} \mathrm{Au},{ }^{202} \mathrm{Hg},{ }^{205} \mathrm{Tl},{ }^{208} \mathrm{~Pb}$, and ${ }^{209} \mathrm{Bi}$. The element contents were calibrated against reference materials of USGS MASS- 1 and USGS GSD-1g using ${ }^{66} \mathrm{Zn}$ as an internal standard for sphalerite and würtzite, and ${ }^{57} \mathrm{Fe}$ for pyrite, respectively [43,44]. All mass fractions for USGS MASS-1 and USGS GSD-1g were taken from the GeoReM base preferred values. The calibration standard was analyzed every 10-12 spots to account for the instrument drift. Data processing was carried out using the Iolite software package [45].

A sphalerite grain of a complex composition $(\mathrm{Zn}, \mathrm{Cd}, \mathrm{Fe})(\mathrm{S}, \mathrm{O}, \mathrm{Se})$ was analyzed by single-crystal X-ray diffractometry on an Oxford Diffraction Xcalibur Gemini diffractometer (Oxford Diffraction Ltd., Wroclaw, Poland), MoK $\alpha, \lambda=0.71073 \AA$ (Novosibirsk State University, Novosibirsk, Russia). Diffraction data were collected by scanning of the $\omega$ angle with a step of $1^{\circ}$ per frame ( $\omega$ scan technique). The data were processed in CrysAlis Pro 171.38.43d (Rigaku-Oxford Diffraction Ltd., Oxfordshire, UK) [46]. Semi-empirical absorption correction was applied using the multi-scan technique.

X-ray powder diffraction analyses of bulk rock samples was performed on an XRD-6000 diffractometer (Shimadzu Corporation, Kyoto, Japan) $\left(\mathrm{CuK} \alpha_{1+2}\right.$ radiation with graphite monochromator), at $4^{\circ}$ to $70^{\circ}$ $2 \Theta$, at a step of $0.5^{\circ}$.

Raman spectra of high-quality sphalerite crystals (reference sample DT-20) were recorded on a Horiba Jobin Yvon LabRAM HR800 spectrometer (Horiba Jobin Yvon S.A.S., Lonjumeau, France). The spectra were excited with two laser lines: a 532-nm green line of a Thorlabs 50-mW power neodymium Nd:YAG laser working at double-harmonic frequency and a Kimmon 325-nm ultraviolet line (UV) of a He-Cd gas laser. The radiated laser power was attenuated with optical filters and the beam power incident onto the sample was about $\sim 0.1 \mathrm{~mW}$. The scattered light was recorded by a 1024-channel Peltier-cooled CCD detector (Andor, Oxford Instruments, international 
company based in Belfast, Northern Ireland) in a region of 70 to $3800 \mathrm{~cm}^{-1}$ at a resolution of $2 \mathrm{~cm}^{-1}$. The excitation was with Olympus objectives at magnifications of $\times 50$ (WD $=0.4 \mathrm{~mm})$ and $\times 100$ $(\mathrm{WD}=0.2 \mathrm{~mm})$ for the green line and with an Olympus objective at $\times 10(\mathrm{WD}=8 \mathrm{~mm})$ and a LOMO objective of $\times 50$ (WD $=0.2 \mathrm{~mm}$ ) for the UV line. The Raman spectra of the selected CM sphalerite crystal $\left(\left(\mathrm{Zn}_{0.96} \mathrm{Fe}_{0.02} \mathrm{Cd}_{0.02}\right)\left(\mathrm{S}_{0.94} \mathrm{O}_{0.05} \mathrm{Se}_{0.01}\right)\right)$ were recorded at different points of the DT-20 sample and deconvolved into Voigt amplitude functions using the Model S506 Interactive PeakFit software (2002 Spectroscopy Software, Canberra Industries, Meriden, CT, USA) [47].

\section{Results}

\subsection{Sedimentary Protoliths and Their TE Hosts}

All Belqa Gr. lithologies in Levant east of the Dead Sea-Jordanian Transform have abnormal enrichment in redox-sensitive elements (RSEs) that largely exceed the absolute contents in most of the organic-rich sediments worldwide. Trace elements are especially abundant and diverse in immature organic-rich siliceous chalk ('oil shale') of the Maastrichtian-Paleocene Muwaqqar Chalk Marl Fm., a unit of the Belqa Gr., in central Jordan (Figure 2) [4,14,18-20,30,48-51]. The Muwaqqar Fm. 'oil shales' were deposited on the southern Neo-Tethys epicontinental shelf in a setting typical of pristine phosphorites, under anoxic/euxinic conditions during intense upwelling. Large amounts of degrading organic matter consumed oxygen from the water column, while reducing conditions were favorable for the accumulation of liptinitic OM, with its share in the chalks reaching 30\% [30,48,52]. Redox-sensitive elements were brought to sediments mainly by a biogenic shuttle. The bulk organic matter consists of type I/II kerogen sulfurized during sulfate reduction. Sulfate-reducing bacteria tended to sulfurize the $\mathrm{OM}$ and finally facilitated prolific precipitation of authigenic sulfides, which accumulated $\mathrm{Zn}, \mathrm{Cd}, \mathrm{Fe}, \mathrm{Ni}, \mathrm{Cu}, \mathrm{Mo}$, and Se [4,14,19,51].

The RSE enrichment is the highest in the basins of western and central Jordan that underwent synsedimentary subsidence $[4,30,48,53]$. The Muwaqqar 'oil shales' from the Siwaqa area, with up to $23 \mathrm{wt} \%$ organic matter and $4.3 \mathrm{wt} \%$ total sulfur (mainly sulfidic), are remarkable for exceptionally high contents of phosphorus and RSE that reach 4695 ppm Zn, 2176 ppm U, 727 ppm V, 496 ppm Mo, 448 ppm Ni, 418 ppm Cr, and 225 ppm Cd. The mean contents of other trace elements are moderate or low: $\leq 60 \mathrm{ppm} \mathrm{Ba}, \leq 0.1 \mathrm{ppm} \mathrm{Bi}, \leq 10 \mathrm{ppm} \mathrm{Co}, \leq 35 \mathrm{ppm} \mathrm{Mn}, \leq 65 \mathrm{ppm} \mathrm{Cu}, \leq 8 \mathrm{ppm} \mathrm{Pb}$, and $\leq 6$ ppm Sb (Table 1) [4,14,30,48]. Metals reside mainly in sulfides: $\mathrm{Zn}-\mathrm{Cd}-(\mathrm{Cu})$ in sphalerite or/and würtzite and Fe-Ni-V-Cu-(Mo) and Se in pyrite. S-rich organic matter is an important carrier of $\mathrm{Ni}, \mathrm{V}$, and sometimes $\mathrm{Cu}$ [14]. These very rocks are the sedimentary protoliths of CM marbles with unique accessory $\mathrm{U}, \mathrm{Cd}, \mathrm{Zn}$, and Ni mineralogy [4,7,9,10,14,18-20,22,23].

'Oil shales' found at the base and in the middle of the Muwaqqar Fm. section are grey laminated fine-grained mainly calcitic rocks with abundant shell clasts (Figure 4). Foraminifera occur mainly in organic-rich sediments with 10 to $20 \mathrm{wt} \%$ total organic carbon. The chalks contain a few percent to 10-15\% biogenic carbonate-fluorapatite, minor percentages of clay minerals (smectite, illite, or rarer kaolinite), quartz occurring as clastic material and/or chalcedony-like matter, as well as opal-A, opal-CT, or microquartz derived from a diatomaceous precursor [14,54,55].

Opaque minerals in the the Muwaqqar Fm. 'oil shales' are authigenic sulfides: predominant würtzite, less-abundant sphalerite (both with 7.6-9.6 $\mathrm{wt} \% \mathrm{Cd}$ ), and sporadic framboidal pyrite (Figure 4) $[4,14,19,30]$. Authigenic Cd-rich sphalerite and würtzite are much more abundant than pyrite, for three main reasons: (i) S-bearing ligands coordinating Cd and Zn in primary OM; (ii) high sulfur in OM; and (iii) low concentrations of reactive iron in bottom sediments [14]. The sulfides were described in detail in an earlier publication in the context of $\mathrm{Zn}$ and $\mathrm{Cd}$ accumulation in bioproductive carbonate sediments [14]. Here, we confine ourselves to a brief summary of their trace-element compositions. EPMA of many sphalerite and würtzite crystals $(\leq 10 \mu \mathrm{m})$ has revealed very high contents of impurities in hexagonal ZnS polymorphs: an average of $8.63 \mathrm{wt} \% \mathrm{Cd}, 0.69 \mathrm{wt} \% \mathrm{Cu}, 0.33 \mathrm{wt} \%$ Fe, and $0.43 \mathrm{wt} \%$ As (Table 2). LA-ICPMS was restricted to a few plates of würtzite larger than $20 \mu \mathrm{m}$. 
Authigenic würtzite has an anomalous TE composition: tens of thousand ppm for Cd; thousands ppm of $\mathrm{Fe}, \mathrm{Cu}$, As, and Sb; and hundreds ppm of $\mathrm{Ni}, \mathrm{Hg}, \mathrm{Pb}, \mathrm{Se}, \mathrm{Mo}$, and $\mathrm{Ag}$ (Table 3). Authigenic pyrite contains tens of thousand ppm of $\mathrm{Cu}$; thousands ppm of As, Se, Mo, and Ag; and hundreds ppm of $\mathrm{Mn}, \mathrm{Co}, \mathrm{Zn}, \mathrm{Pb}$, and $\mathrm{Tl}$. These TE resources were redistributed and partitioned between newly-formed $\mathrm{CM}$ sulfides during high-temperature alteration of the 'oil shales'.
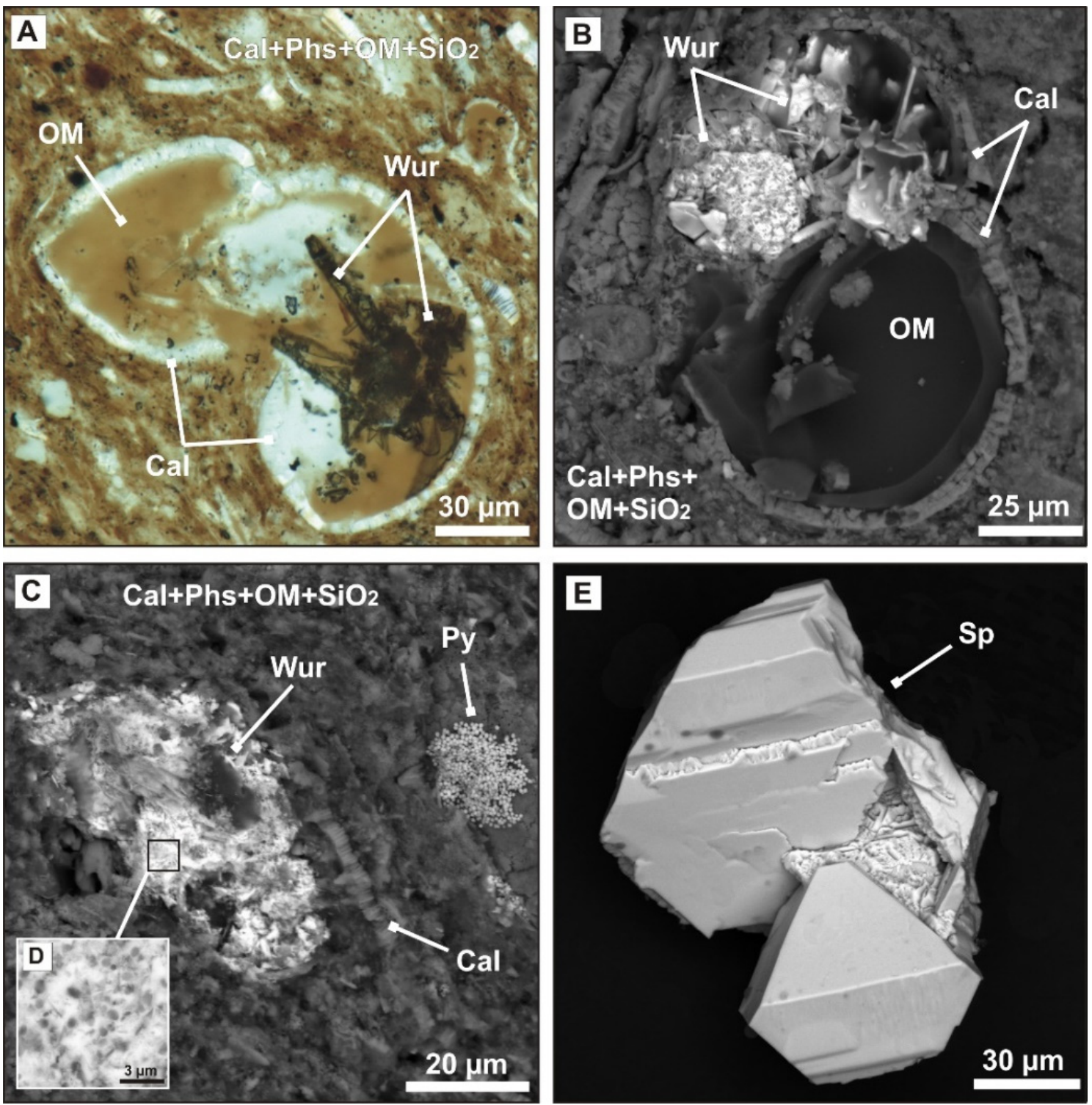

Figure 4. Cd-würtzite, Cd-sphalerite, and associated minerals in bituminous phosphatic chalk ('oil-shale') of the lower Muwaqqar Chalk Marl Formation. (A) Platy Cd-rich würtzites clustered inside foraminiferal chambers in a biomicritic matrix composed of calcite, silica, disseminated organic matter, and pristine phosphorite. (B) Foraminifera chambers filled with S-rich solid organic matter (type-II kerogen). Cd-rich würtzites occur as platy crystals and microcrystals inside chambers. (C) Cd-rich würtzite microcrystals and framboidal pyrite in biomicritic matrix. (D) Micrometer platy Cd-rich würtzite crystallites intimately intergrown with diatom frustules and radiolaria spicules (opal-CT). (E) Simple twinned pseudooctahedral sphalerite. Optical images in plane-polarized light (A) and BSE images $(\mathbf{B}-\mathbf{E})$. Cal = calcite, $\mathrm{OM}=$ organic matter, $\mathrm{Phs}=$ phosphorite, $\mathrm{Py}=$ pyrite; $\mathrm{Sp}=$ sphalerite, Wur $=$ wurtzite. 
Table 2. Average composition of sphalerite and würtzite from sedimentary rocks ('oil shales') of the Muwaqqar Chalk Marl Formation (SEM-EDS and EPMA data, wt\%).

\begin{tabular}{ccccccccc}
\hline \multirow{2}{*}{$\begin{array}{c}\text { Mineral } \\
\text { Sample }\end{array}$} & \multicolumn{4}{c}{ Sphalerite } & \multicolumn{4}{c}{ Würtzite } \\
\cline { 2 - 9 } DOS and OSB (n= 24) & \multicolumn{3}{c}{ DOS (n = 27) } \\
\hline & Mean & $S$ & Min & Max & Mean & $S$ & Min & Max \\
\hline $\mathrm{Zn}$ & 58.20 & 0.26 & 57.80 & 58.51 & 58.01 & 0.99 & 56.26 & 59.18 \\
$\mathrm{Cd}$ & 9.12 & 0.24 & 8.84 & 9.45 & 8.63 & 0.76 & 7.63 & 9.96 \\
$\mathrm{Fe}$ & 0.27 & 0.25 & $<0.10$ & 0.50 & 0.33 & 0.21 & $<0.10$ & 0.70 \\
$\mathrm{Cu}$ & $<0.15$ & - & $<0.15$ & $<0.15$ & 0.69 & 0.15 & 0.42 & 0.91 \\
$\mathrm{~S}$ & 32.08 & 0.06 & 31.99 & 32.15 & 32.04 & 0.16 & 31.82 & 32.33 \\
Total & 99.67 & & & & 99.70 & & & \\
\hline
\end{tabular}

$\mathrm{Mn}, \mathrm{Ni}(<0.10 \mathrm{wt} \%)$, and Se $(<0.15 \mathrm{wt} \%)$ are below detection limits. Würtzite also contains As (mean $0.43 \mathrm{wt} \%$, $\mathrm{S} 0.18 \mathrm{wt} \%$, $\min 0.21 \mathrm{wt} \%$, max $0.74 \mathrm{wt} \%$ ) and $\mathrm{Sb}$ (mean $0.13 \mathrm{wt} \%, \mathrm{~S} 0.05 \mathrm{wt} \%, \min <0.09 \mathrm{wt} \%, \max 0.20 \mathrm{wt} \%$ ). $\mathrm{n}=$ number of analyses, Mean = mean value $S=$ standard deviation, Min $=$ minimum value, Max $=$ maximum value.

Table 3. Average trace-element composition (ppm) of sphalerite from spurrite-fluorapatite marbles (Tulul al Hammam area), compared with Fe- and Zn sulfides from 'oil shales' (Muwaqqar Chalk Marl Formation). LA-ICPMS data.

\begin{tabular}{|c|c|c|c|c|}
\hline \multirow{2}{*}{$\begin{array}{c}\text { Rock Type } \\
\text { Sample }\end{array}$} & \multicolumn{2}{|c|}{ 'Oil Shales' } & \multicolumn{2}{|c|}{ Spurrite-Fluorapatite Marbles } \\
\hline & DOS-1 & DOS-1 & DT-20 & DT-25 \\
\hline Mineral & Pyrite & Würtzite & Sphalerite & Sphalerite \\
\hline $\mathrm{n}$ & 6 & 5 & 16 & 3 \\
\hline \multicolumn{5}{|l|}{ Element } \\
\hline V & 1554 & 2.93 & bdl & bdl \\
\hline $\mathrm{Mn}$ & 647 & 7.3 & 1565 & 355 \\
\hline $\mathrm{Co}$ & 120 & bdl & 124 & 66 \\
\hline $\mathrm{Ni}$ & 4990 & 230 & 220 & 265 \\
\hline $\mathrm{Cu}$ & 34,340 & 7760 & 11.1 & 26 \\
\hline $\mathrm{Ga}$ & bdl & 202 & 0.45 & 7.9 \\
\hline $\mathrm{Ge}$ & 2.75 & 381 & bdl & bdl \\
\hline As & 1343 & 2255 & bdl & bdl \\
\hline $\mathrm{Se}$ & 6535 & 282 & 4440 & 13,910 \\
\hline Mo & 1332 & 606 & bdl & bdl \\
\hline $\mathrm{Ag}$ & 1103 & 645 & 0.42 & 0.53 \\
\hline $\mathrm{Cd}$ & 114 & 66,200 & 18,100 & 9240 \\
\hline In & bdl & 0.69 & 4.51 & 2.44 \\
\hline $\mathrm{Sb}$ & 41.2 & 2145 & 1.19 & 3.03 \\
\hline $\mathrm{Te}$ & bdl & bdl & bdl & bdl \\
\hline $\mathrm{Au}$ & 0.3 & bdl & bdl & bdl \\
\hline $\mathrm{Hg}$ & bdl & 345 & 132 & 226 \\
\hline $\mathrm{Tl}$ & 163 & 5.2 & bdl & bdl \\
\hline $\mathrm{Pb}$ & 269 & 604 & bdl & 0.9 \\
\hline $\mathrm{Bi}$ & 5.5 & 5.3 & bdl & bdl \\
\hline
\end{tabular}

$\mathrm{bdl}=$ below detection limit; $\mathrm{n}=$ number of samples. Concentration of $\mathrm{Zn}$ in pyrite is $280 \mathrm{ppm}$.

\subsection{Zn-Rich Marbles: Occurrence, Rock Chemistry, and Mineral Assemblages}

The Tulul al Hammam sphalerite-bearing marbles are varicolored (mostly dark-shaded), foliated, massive, and microcrystalline $(\leq 50-200 \mu \mathrm{m})$. The amount of sphalerite (Figure 5; Table 4) varies from single $\leq 2-10 \mu \mathrm{m}$ particles in slightly altered greenish marbles (samples labeled as $\mathrm{TH}$ ) to clusters of numerous crystals reaching $200 \mu \mathrm{m}$ in fresh deep-brown fluorapatite marbles (samples labeled as DT) (Figure 3). These metasedimentary rocks share similarity in bulk chemistry, with predominant $\mathrm{CaO}(51.5-55.0 \mathrm{wt} \%)$ and commensurate contents of $\mathrm{SiO}_{2}$ and $\mathrm{P}_{2} \mathrm{O}_{5}(3.40-4.85$ and 3.74-4.54 wt\%, respectively). Loss on ignition (LOI) is 29.1 to $34.1 \mathrm{wt} \%$, mainly due to $\mathrm{CO}_{2}$. The contents of other major elements are quite low, being within $2.28 \mathrm{wt} \% \mathrm{Al}_{2} \mathrm{O}_{3}, 2.28 \mathrm{wt} \% \mathrm{Fe}_{2} \mathrm{O}_{3}, 0.65 \mathrm{wt} \% \mathrm{MgO}, 0.21 \mathrm{wt} \%$ $\mathrm{Na}_{2} \mathrm{O}$, and $0.06 \mathrm{~K}_{2} \mathrm{O}$. Bulk sulfur (determined as $\mathrm{SO}_{3}$ ) is commonly $0.4-0.5 \mathrm{wt} \%$. 
The DT samples (Table 1) stand out against similar marbles of the same locality in Zn enrichment times greater than in the TH samples (1091 to 1660 ppm against 230-470 ppm Zn) which include only sporadic fine grains of $(\mathrm{Zn}, \mathrm{Cd}) \mathrm{S}$ or $(\mathrm{Cd}, \mathrm{Zn})(\mathrm{S}, \mathrm{Se})$ solid solutions. Other RSE concentrations in the DT samples are also relatively high and reach 1152 ppm V, 42.6 ppm Ag, 329 ppm Cu, 684 ppm Ni, 81.9 ppm As, 503 ppm Se, 70.3 ppm Mo, 75 ppm Cd, and 24.0 ppm U. Most of the samples with higher TE loading show an uneven distribution of $\mathrm{Zn}, \mathrm{Cd}, \mathrm{Ni}, \mathrm{Ag}, \mathrm{U}$, and Se and enclose excretions of the corresponding phases (Figure $5 \mathrm{~A}, \mathrm{C}$ ). In fact, the CM rocks display a snapshot of original TE hosts left by the annealing of sediments: OM, pyrite, and $\mathrm{ZnS}$ polymorphs.
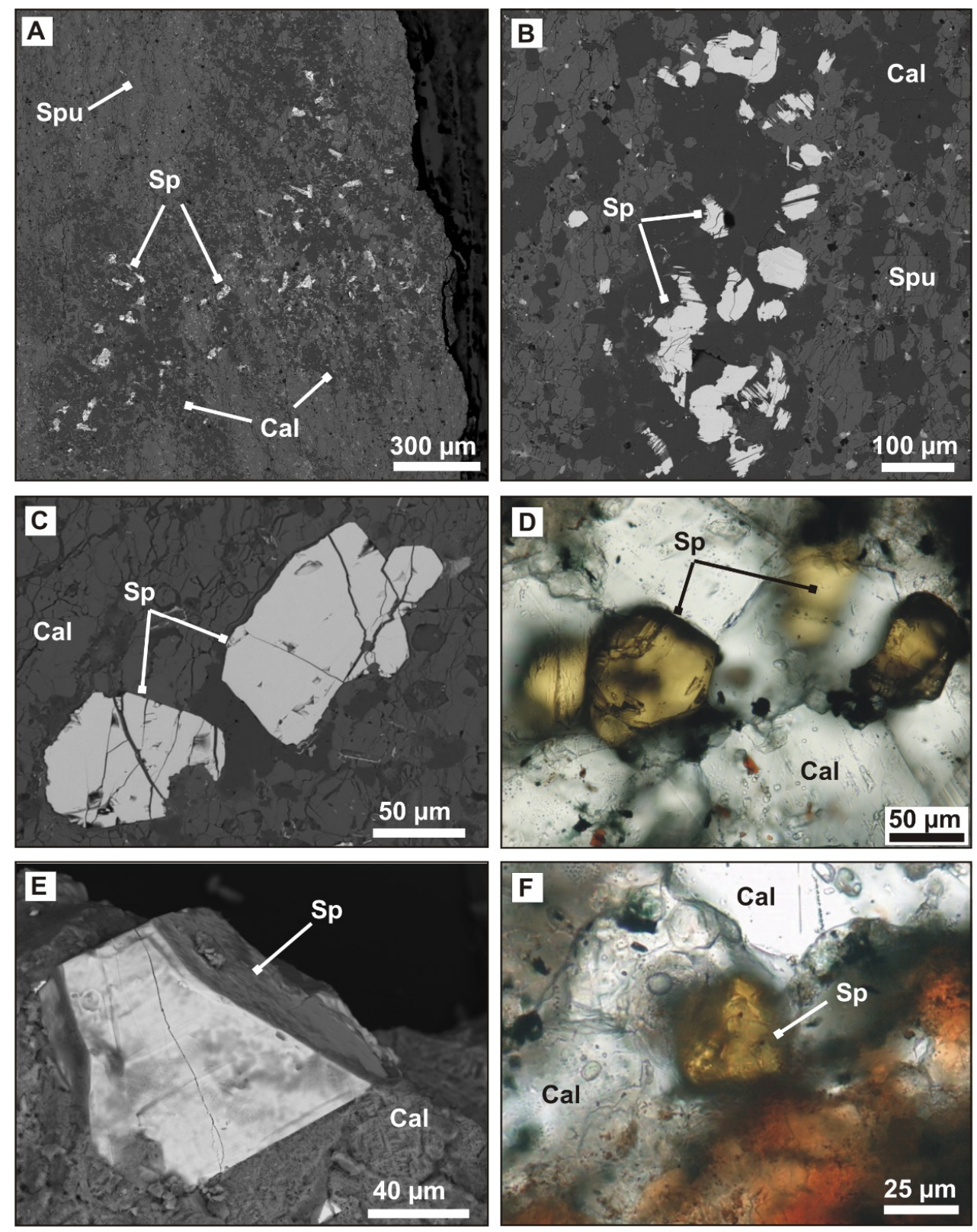

Figure 5. Sphalerite from medium-temperature combustion metamorphic spurrite-fluorapatite marbles (Tulul al Hammam area, central Jordan). (A,B) Numerous large sphalerite crystals inside calcite-rich layer. (C-F) Tetrahedral or pseudo-octahedral sphalerite twins in a calcite matrix. Sample DT-25 (A), sample DT-26 (B), sample DT-20 (C-F). Optical images in plane-polarized light (D,F) and BSE images $(\mathrm{A}-\mathrm{C}, \mathrm{E}) . \mathrm{Cal}=$ calcite, $\mathrm{Sp}=$ sphalerite, $\mathrm{Spu}=$ spurrite. 
Most of the studied marbles consist of $\sim 75-90 \%$ calcite, up to $15 \%$ carbonate-fluorapatite, $\leq 5 \%$ spurrite $\left(\mathrm{Ca}_{5}\left(\mathrm{SiO}_{4}\right)_{2}\left(\mathrm{CO}_{3}\right)\right)$, and $\leq 3 \%$ brownmillerite $\left(\mathrm{Ca}_{2}\left(\mathrm{Fe}_{1-x} \mathrm{Al}_{x}\right)_{2} \mathrm{O}_{5}\right)$, as well as sporadic grains of $\mathrm{Ca}$ ferrites, mayenite supergroup minerals, $\mathrm{Zn}$-rich periclase, tululite, and accessory phases of $\mathrm{Zn}, \mathrm{Cd}, \mathrm{Ni}, \mathrm{Cu}, \mathrm{Ag}$, and $\mathrm{U}$ (Table 4). The spurrite and fluorapaite percentages are as high as $\sim 15 \%$ in sample DT-25, which contains $10.03 \mathrm{wt} \% \mathrm{SiO}_{2}$ (Figure 5A). Fluorapatite from sulfide-rich layers in the marbles contains no more than $1.85 \mathrm{wt} \% \mathrm{SO}_{3}$, while average contents of $\mathrm{SiO}_{2}$ are $4.7-6.5 \mathrm{wt} \%$. Marbles with abundant $\mathrm{Zn}$-bearing oxides contain fluorapatites enriched in both $\mathrm{SiO}_{2}(5.9-7.8 \mathrm{wt} \%)$ and $\mathrm{SO}_{3}(2.8-5.3 \mathrm{wt} \%)$ (Figure 6; Table 5).

Table 4. Mineral assemblages of Zn-enriched Jordanian combustion metamorphic rocks—phosphate marbles with primarily Zn-bearing oxide and sulfide mineralization, Tulul al Hammam area. EPMA, SEM EDS, and XRD data.

\begin{tabular}{|c|c|c|c|c|}
\hline Sample/Rock Type & Main Phases & Minor Phases & Accessory Phases & Alteration Products \\
\hline $\begin{array}{c}\text { DT-20 } \\
\text { Spurrite-fluorapatite marble } \\
\text { Zn-1430 ppm; } \\
\text { Cd-52.7 ppm } \\
\text { Zn/Cd = 27.1 }\end{array}$ & $\begin{array}{c}\text { Calcite, } \\
\text { * Fluorapatite } \\
\left(\mathrm{SiO}_{2}-4.69 \mathrm{wt} \%\right. \\
\left.\mathrm{SO}_{3}-1.13 \mathrm{wt} \%\right)\end{array}$ & Spurrite & $\begin{array}{c}\text { Sphalerite, } \\
\text { Tululite, } \\
\text { Zincite, } \\
\text { Periclase }(\mathrm{Zn}-, \mathrm{Cu}-, \mathrm{Ni}-\text { rich }), \\
\text { (Ca,Cd)O, } \\
\text { Brownmillerite }(\mathrm{Cr}, \mathrm{Ti}, \\
\text { Zn-rich), } \\
\text { Ca ferrite, } \\
\text { Fe, Ni, Cu sulfides, } \\
\text { Ca-Fe-S-O compounds, } \\
\text { Cerianite }(\mathrm{Ce}, \mathrm{Th}) \mathrm{O}_{2}, \\
\text { Lime }(\mathrm{CaO}), \\
\text { Lakargiite }\left(\mathrm{CaZrO} \mathrm{ra}_{3}\right)\end{array}$ & $\begin{array}{c}\text { CSHs (trace), } \\
\text { Chlormayenite, } \\
\text { Portlandite }\left(\left(\mathrm{Ca}(\mathrm{OH})_{2}\right),\right. \\
\text { Si- and F-bearing hydrated } \\
\text { CaO-UO } \mathrm{UO}_{3} \text { compounds }\end{array}$ \\
\hline $\begin{array}{c}\text { DT-25 } \\
\text { Fluorapatite-spurrite-marble } \\
\text { Zn-1134 ppm; } \\
\text { Cd-41.4 ppm; } \\
\text { Se-503 ppm } \\
\text { Zn/Cd = 27.4 }\end{array}$ & $\begin{array}{c}\text { Calcite, } \\
\text { Spurrite } \\
\text { (Na-, P-bearing), } \\
\text { * Fluorapatite } \\
\left(\mathrm{SiO}_{2}-4.95 \mathrm{wt} \% ;\right. \\
\left.\mathrm{SO}_{3}-1.85 \mathrm{wt} \%\right)\end{array}$ & $\begin{array}{c}\text { Brownmillerite (Ti, Zn, } \\
\text { Cr-bearing) }\end{array}$ & $\begin{array}{c}\text { Sphalerite } \\
\text { (Na,K,Ba)-Zn sulfide, } \\
\text { Ca-Fe-Cu-S-Se-O } \\
\text { compounds } \\
\text { Ni-Se-S compound (Ni(Se,S)) } \\
\text { Ni-S-Se compound } \\
\text { (Ni(S,Se)), } \\
\text { Greenockite }(\mathrm{Cd}, \mathrm{Zn})(\mathrm{S}, \mathrm{Se}), \\
\text { Cadmoselite, CdSe (scare), } \\
\text { Naumannite }\left(\mathrm{Ag}_{2} \mathrm{Se}\right) \text { (scare), } \\
\text { Srebrodolskite }(\mathrm{Zn}-\text { bearing), } \\
\text { Vorlanite }\left(\mathrm{CaUO} \mathrm{O}_{4}\right) \text { (scare), } \\
\text { Nabimusaite, } \\
\text { Anhydrite (scare), } \\
\text { Fluormayenite-Chlormayenite } \\
\text { ss. (scare) }\end{array}$ & $\begin{array}{c}\text { Hydrocalumite, } \\
\text { Si-and F-bearing hydrated } \\
\text { CaO-UO } \mathrm{UO}_{3} \text { compounds }\end{array}$ \\
\hline $\begin{array}{c}\text { DT-26 } \\
\text { Spurrite-fluorapatite marble } \\
\text { Zn-1440 ppm; } \\
\text { Cd-39 ppm } \\
\text { Zn/Cd = 36.9 }\end{array}$ & $\begin{array}{c}\text { Calcite, } \\
{ }^{*} \text { Fluorapatite } \\
\left(\mathrm{SiO}_{2}-4.30 \mathrm{wt} \%\right. \\
\left.\mathrm{SO}_{3}-0.49 \mathrm{wt} \%\right)\end{array}$ & Spurrite & $\begin{array}{c}\text { Sphalerite, } \\
\text { Na-Zn sulfide } \\
\left((\mathrm{Na}, \mathrm{K}, \mathbf{B a})_{2} \mathbf{Z n}_{4} \mathrm{~S}_{5}\right), \\
\text { Ni-Se-S-(Zn, } \mathbf{C u}) \\
\text { compound, } \\
\text { Ca-Fe-Cu-Ni-S-O } \\
\text { compounds, } \\
\text { Brownmillerite ( } \leq 0.45 \mathrm{wt} \% \\
\text { ZnO) (scare), } \\
\text { Bartonite (scare), } \\
\text { Ag-Se compound (scare), } \\
\left.\text { Rasvumite (KFe } \mathrm{K}_{3}\right) \text { (scare), } \\
\text { Ca-Ti ferrite, } \\
\text { Vorlanite, } \\
\text { Tylleite (scare), } \\
\text { Fluormayenite (scare), } \\
\text { Lakargiite (scare) }\end{array}$ & $\begin{array}{c}\text { Hydrocalumite, } \\
\text { Bulfonteinite } \\
\left(\mathrm{Ca}_{2} \mathrm{SiO}_{2}(\mathrm{OH}, \mathrm{F})_{4}\right), \\
\mathrm{CSHs}(\text { trace), } \\
\text { Portlandite }\left(\mathrm{Ca}(\mathrm{OH})_{2}\right), \\
\text { Cl-bearing hibschite (?) }\end{array}$ \\
\hline $\begin{array}{c}\text { TH-74 } \\
\text { Altered marble } \\
\text { Zn-333 ppm; } \\
\text { Cd-75 ppm } \\
\text { Zn/Cd = 4.44 }\end{array}$ & Calcite & $\begin{array}{c}\text { * Fluorapatite } \\
\left(\mathrm{SiO}_{2}-5.30 \mathrm{wt} \% ;\right. \\
\left.\mathrm{SO}_{3}<0.3 \mathrm{wt} \%\right), \\
\text { Brownmillerite }(\mathrm{Cr}, \mathrm{Ti}, \\
\text { Zn-bearing })\end{array}$ & 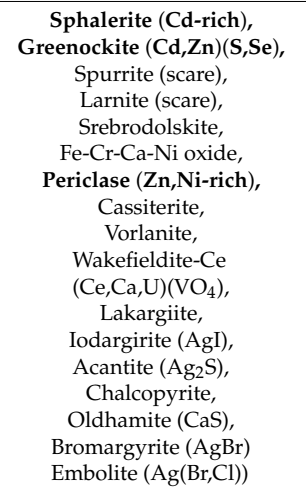 & $\begin{array}{c}\text { CSHs, } \\
\text { Zeolites, } \\
\text { Chlormayenite, } \\
\text { Fe hydroxides, } \\
\text { Gypsum, } \\
\text { Portlandite, } \\
\text { Carnotite }\left(\mathrm{K}_{2}\left(\mathrm{UO}_{2}\right)_{2} \mathrm{~V}_{2} \mathrm{O}_{8} \cdot 3\left(\mathrm{H}_{2} \mathrm{O}\right) \text {, }\right. \\
\text { Tyuyamunite or Metatyuyamunite } \\
\left(\mathrm{Ca}\left(\mathrm{UO}_{2}\right)_{2} \mathrm{~V}_{2} \mathrm{O}_{8} \cdot 5-8 \mathrm{H}_{2} \mathrm{O}\right), \\
\text { Strelkinite } \\
\left(\mathrm{Na}_{2}\left(\mathrm{UO}_{2}\right)_{2} \mathrm{~V}_{2} \mathrm{O}_{8} \cdot 6 \mathrm{H}_{2} \mathrm{O}\right), \\
\mathrm{Si} \text {-and } \mathrm{F}-\text { bearing hydrated } \\
\mathrm{CaO}-\mathrm{UO}_{3} \text { compounds, } \\
\text { Plattnerite }(?)\left(\mathrm{PbO}_{2}\right) \\
\text { Crocoite }\left(\mathrm{PbCrO}_{4}\right), \\
\text { Chromatite }\left(\mathrm{CaCrO}_{4}\right)\end{array}$ \\
\hline
\end{tabular}


Table 4. Cont.

\begin{tabular}{|c|c|c|c|c|}
\hline Sample/Rock Type & Main Phases & Minor Phases & Accessory Phases & Alteration Products \\
\hline $\begin{array}{c}\text { TH-79 } \\
\text { Fluorapatite marble } \\
\text { Zn-231 ppm; } \\
\text { Cd }<3 \text { ppm } \\
\text { Zn/Cd > 77 }\end{array}$ & $\begin{array}{c}\text { Calcite, } \\
\text { * Fluorapatite } \\
\left(\mathrm{SiO}_{2}-6.5 \mathrm{wt} \%\right. \\
\left.\mathrm{SO}_{3}-4.83 \mathrm{wt} \%\right)\end{array}$ & & $\begin{array}{c}\text { Sphalerite, } \\
\mathrm{Ca}_{2} \mathrm{UO}_{5}(?), \\
\text { Baritocelestine, } \\
\text { Srebrodolskite, } \\
\text { Bartonite, } \\
\text { Murunskite }\left(\mathrm{K}_{2} \mathrm{Cu}_{3} \mathrm{FeS}_{4}\right), \\
\text { Chalcopyrite, } \\
\text { Oldhamite }\end{array}$ & $\begin{array}{l}\text { CSHs, } \\
\text { Aragonite, } \\
\text { Gypsum, } \\
\text { Opal, } \\
\text { Portlandite, } \\
\text { Chromatite }\end{array}$ \\
\hline $\begin{array}{c}\text { TH-11 } \\
\text { Fluorapatite marble } \\
\text { Zn-470 ppm; } \\
\text { Cd-28 ppm } \\
\text { Zn/Cd = 16.8 }\end{array}$ & $\begin{array}{c}\text { Calcite, } \\
{ }^{*} \text { Fluorapatite } \\
\left(\mathrm{SiO}_{2}-5.47 \mathrm{wt} \% ;\right. \\
\left.\mathrm{SO}_{3}-1.64 \mathrm{wt} \%\right)\end{array}$ & & $\begin{array}{c}\text { Sphalerite, } \\
\text { Zincite, } \\
(\text { Ca,Cd)O, } \\
\text { Lakargiite, } \\
\text { Bunsenite (NiO), } \\
\text { Lime, } \\
\text { Baritocelestine, } \\
\text { Ag }^{0} \text {, Acantite, } \\
\text { Iodargirite (AgI), } \\
\text { Galena }\end{array}$ & $\begin{array}{c}\text { CSHs, } \\
\text { Chlorite (?), } \\
\text { Tyuyamunite or } \\
\text { Metatyuyamunite, }\end{array}$ \\
\hline $\begin{array}{c}\text { TH-52 } \\
\text { Fluorapatite marble altered } \\
\mathrm{Zn}-329 \mathrm{ppm} ; \\
\mathrm{Cd}-25.9 \mathrm{ppm} \\
\mathrm{Zn} / \mathrm{Cd}=12.7\end{array}$ & $\begin{array}{c}\text { Calcite } \\
{ }^{*} \text { Fluorapatite } \\
\left(\mathrm{SiO}_{2}-5.65 \mathrm{wt} \% ;\right. \\
\left.\mathrm{SO}_{3}-4.15 \mathrm{wt} \%\right)\end{array}$ & $\begin{array}{c}\text { * Fluorellestadite } \\
\left(\mathrm{SiO}_{2}-10.24 \mathrm{wt} \%\right. \\
\left.\mathrm{SO}_{3}-12.69 \mathrm{wt} \%\right) \\
\text { Fluorite, } \\
\text { Brownmillerite, } \\
\text { Larnite (hydrated), } \\
\text { Bredigite (?) (hydrated) } \\
\text { Chlormayenite }\end{array}$ & $\begin{array}{c}\text { Sphalerite, } \\
\text { Fe-Zn spinel, } \\
\text { Srebrodolskite, } \\
\text { Lakargiite, } \\
\text { Barite, } \\
\text { Baritocelestine } \\
\text { Galena, } \\
\text { Pyrrhotite, } \\
\text { Chalcopyrite, } \mathrm{NaCl}\end{array}$ & $\begin{array}{c}\text { CSHs, } \\
\text { Volkonskoite, } \\
\text { Talc, } \\
\text { Chlorite (?), } \\
\text { Carnotite, } \\
\text { Tyuyamunite, } \\
\text { Strelkinite, } \\
\text { Mn hydroxides (Ni- and Zn-rich), } \\
\mathrm{SnCl}_{4}(?)\end{array}$ \\
\hline
\end{tabular}

Main phases (>10 vol\%), minor phases (5-10 vol\%) and accessories (<3 vol\%). *-mean contents of $\mathrm{SiO}_{2}$ and $\mathrm{SO}_{3}$ determined in carbonate-fluorapatite and fluorellestadite by EDS SEM and electron microprobe technics. CSHs-Ca silicate hydrates. Zn- and/or Cd-bearing minerals are in bold. The composition of the samples TH-11 and TH-74 were partially characterized in $[18,19]$; other samples this study.

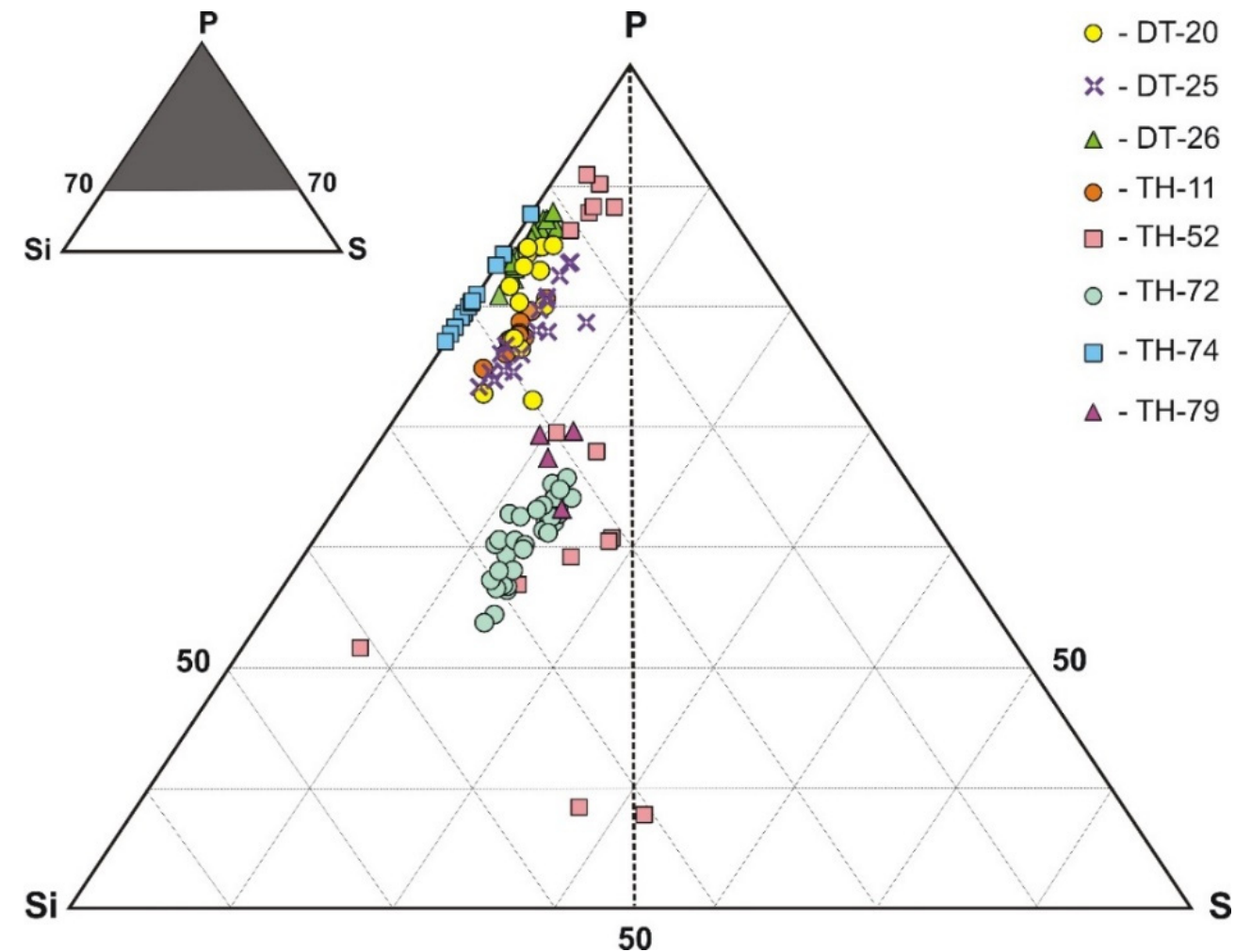

Figure 6. Phosphorus-rich part of the Si-P-S diagram showing the composition (in atoms per formula unit, apfu) of fluorapatite and fluorellestadite from CM marbles (Tulul al Hammam area). In the samples DT-25 and DT-26, carbonate- fluorapatite was identified by Raman spectroscopy. 
Table 5. Mineral assemblage in the reference sample of Zn-enriched spurrite-fluorapatite marble with abundant primary Zn-bearing oxides, Tulul al Hammam area. EPMA, SEM EDS, and XRD data.

\begin{tabular}{|c|c|c|c|c|}
\hline $\begin{array}{c}\text { Sample/Rock } \\
\text { Type }\end{array}$ & Main Phases & Minor Phases & Accessory Phases & Alteration Products \\
\hline
\end{tabular}

Main phases are $>10 \mathrm{vol} \%$; minor phases are 5 to $10 \mathrm{vol} \%$; accessories are $<3 \mathrm{vol} \%$. * mean composition of fluorapatite. $\mathrm{Zn}$ - and/or Cd-bearing minerals are in bold. Sample characteristic after [18,19] and this study.

The distribution of brownmillerite, the most common opaque mineral in the Tulul al Hammam marbles, is apparently controlled by uneven patterns of authigenic pyrite and clayey matter in primary sediments. Brownmillerite is the principal host of $\mathrm{Fe}^{3+}, \mathrm{Al}$, and $\mathrm{Zn}$ in low-Zn spurrite-fluorapatite-calcite layers where it contains up to $3.8 \mathrm{wt} \% \mathrm{ZnO}$. The $\mathrm{Zn}$ contents are similar (2.4-3.8 wt $\%$ ) in brownmillerite from marbles rich in Zn-bearing oxides (zincite, tululite, $\mathrm{Zn}$-rich periclase) but are below $0.5 \mathrm{wt} \%$ in that coexisting with sphalerite. Other $\mathrm{Zn}$-bearing oxides found coexisting with sphalerite are $\mathrm{Zn}$-rich periclase (22-23.5 $\mathrm{wt} \% \mathrm{ZnO})$, scarce grains of $\mathrm{Zn}$-bearing $(\mathrm{Ca}, \mathrm{Cd}) \mathrm{O}$ and/or tululite, but no zincite was detected in any sample. Frequently, $\mathrm{Zn}$ sulfide and oxide accessories are unevenly distributed in samples. Thin laminas or lenses enriched in fluorapatite-fluorellestadite solid solutions enclose tululite, $\mathrm{Zn}$-rich periclase, and $(\mathrm{Ca}, \mathrm{Cd}) \mathrm{O}$, while sphalerite may be abundant in adjacent layers with fluorapatite excretions, as in sample DT-20 bearing numerous large sphalerite crystals. Sphalerite occurs in Zn-rich marbles as transparent $\leq 200 \mu \mathrm{m}$ pseudo-octahedral simple twins or tetrahedral crystals of deep yellow-brownish colors (DT-20), subhedral grains (DT-26), or complex intergrowths and exsolution lamelli with $\mathrm{Ni}, \mathrm{Cd}, \mathrm{Cu}$, and Ag sulfides and/or selenides (DT-25) (Figures 5, 7 and 8).

The marbles with the greatest TE loading (samples DT-20, DT-25 and DT-26) store a whole collection of mineralogical rarities (Tables 4 and 6-8). The rocks include diverse $\mathrm{Fe}, \mathrm{Ni}, \mathrm{Cu}$, and $\mathrm{Ag}$ chalcogenides that coexist with sphalerite and occur as complex micrometer aggregates exsolved from primary high-temperature complex sulfides, sulfo-selenides, and/or oxychalcogenides. They are rasvumite (with $\sim 3 \mathrm{wt} \% \mathrm{Tl}$ and $1.1 \mathrm{wt} \% \mathrm{Rb}$ ), bartonite ( $0.5 \mathrm{wt} \% \mathrm{Rb}$ and $2.7-2.9 \mathrm{wt} \% \mathrm{Se}$ ), naumannite $\left(\mathrm{Ag}_{2} \mathrm{Se}\right)$, cadmoselite (CdSe), a potentially new mineral of $(\mathrm{Na}, \mathrm{K}, \mathrm{Ba})-\mathrm{Zn}$ sulfide $\left((\mathrm{Na}, \mathrm{K}, \mathrm{Ba})_{2} \mathrm{Zn}_{4} \mathrm{~S}_{5}\right)$, Ni selenides, and sulfoselenides, as well as slightly hydrated complex Ca-Fe-Cu-S-Se-O compounds (probably oxysulfides and oxyselenides). The morphology, phase relations, and distributions of elements in coexisting minerals are illustrated in Tables 6 and 7 and in elemental maps (Figures 7 and 8), but many fine phases can hardly be identified as mineral species because they are intimately intergrown.

Akin to the majority of CM rocks in the Tulul al Hammam area $[14,18,19]$, sphalerite marbles contain a set of trace elements $(\mathrm{Cd}, \mathrm{Ce}, \mathrm{Cu}, \mathrm{Ni}, \mathrm{Th}, \mathrm{U}, \mathrm{Zn}, \mathrm{Zr}, \mathrm{Sn})$ accumulated in simple or double oxide accessories (commonly $<10 \mu \mathrm{m}$, up to 20-60 $\mu \mathrm{m}$ ). These accessories include periclase (with $22-23.5 \mathrm{wt} \%$ $\mathrm{ZnO}, 5.0-6.3 \mathrm{wt} \% \mathrm{NiO}$, and $2.5-3.4 \mathrm{wt} \% \mathrm{CuO})$, intermediate members of the ( $\mathrm{Ca}, \mathrm{Cd}) \mathrm{O}$ solid solutions $(0.3-0.9 \mathrm{wt} \% \mathrm{ZnO})$, tululite (23.7 $\mathrm{wt} \% \mathrm{ZnO}$ on average), cassiterite, cerianite $(\mathrm{Ce}, \mathrm{Th}) \mathrm{O}_{2}$, vorlanite $\left(\mathrm{CaUO}_{4}\right)$, and lakargiite $\left(\mathrm{CaZrO}_{3}\right)$. Other accessory minerals are scanty lime $(\mathrm{CaO})$, oldhamite $(\mathrm{CaS})$, srebrodolskite, and Fe-Zn spinel (Table 8). Zincite was found only in sample DT-20 with the highest $\mathrm{Zn}$ contents, where it is restricted to sulfide-free layers 

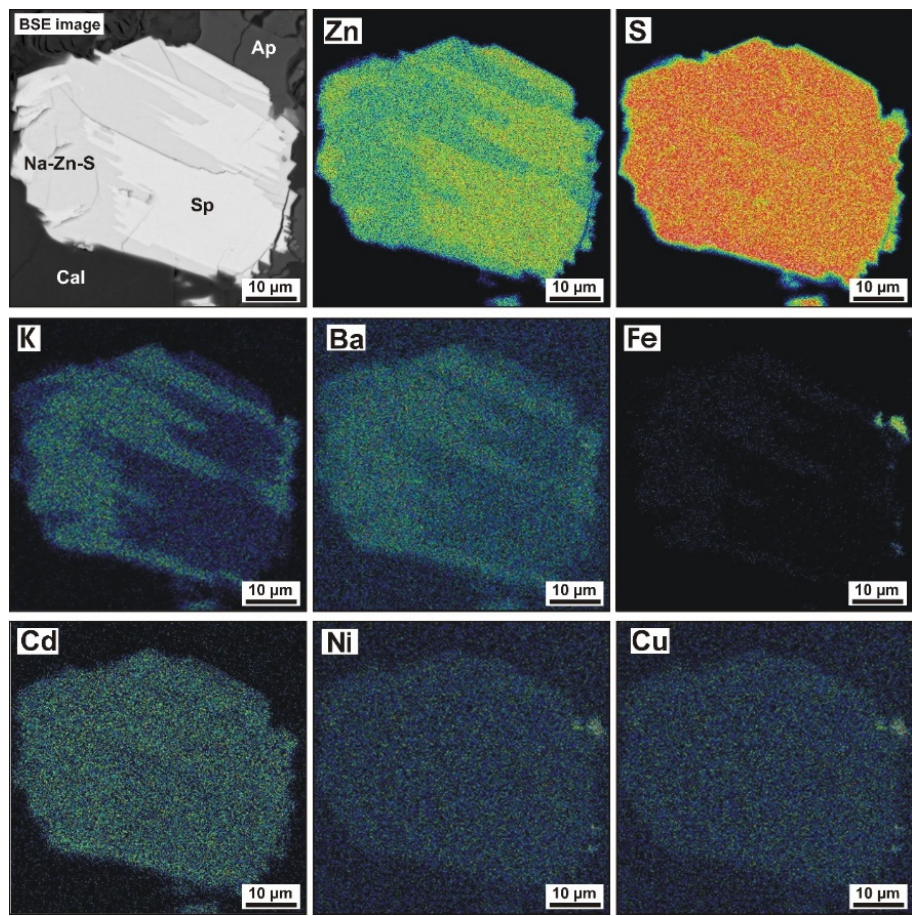

$\mathrm{Cu}$

Figure 7. BSE image and elemental maps ( $\mathrm{Zn}, \mathrm{S}, \mathrm{K}, \mathrm{Ba}, \mathrm{Fe}, \mathrm{Cd}, \mathrm{Ni}, \mathrm{Cu}$ ) showing intimately intergrown sphalerite and $\mathrm{Na}(\mathrm{K}, \mathrm{Ba})-\mathrm{Zn}$ sulfide. Sample DT-26. Ap = fluorapatite, $\mathrm{Cal}=$ calcite, $\mathrm{Na}-\mathrm{Zn}-\mathrm{S}=\mathrm{Na}(\mathrm{K}, \mathrm{Ba})-\mathrm{Zn}$ sulfide, $\mathrm{Sp}=$ sphalerite. The Na map is not shown here due to Zn-Na overlapping.
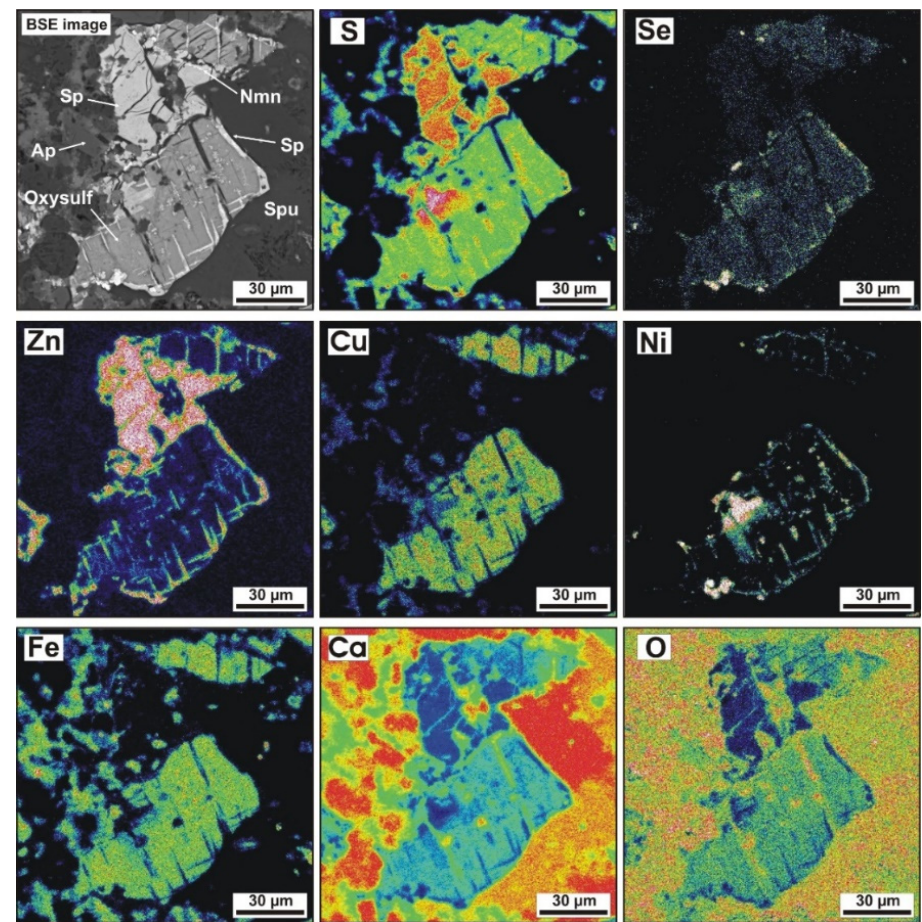

Figure 8. BSE image and elemental maps ( $\mathrm{S}, \mathrm{Se}, \mathrm{Zn}, \mathrm{Cu}, \mathrm{Ni}, \mathrm{Fe}, \mathrm{Ca}, \mathrm{O})$ showing sphalerite coexisting with complex Fe-Ca-Cu-S-O-Se-bearing compounds. Sample DT-25. Ap = fluorapatite, $\mathrm{Cal}=$ calcite, $\mathrm{Nmn}=$ naumannite, $\mathrm{Oxysulf}=$ complex Ca-Fe-Cu-S-Se-O compounds (oxychalcogenides?), $\mathrm{Sp}=$ sphalerite, $\mathrm{Spu}=$ spurrite. 
Table 6. BSE images and EDS compositions ( $\mathrm{wt} \%$ ) of sphalerite and associated opaque minerals from the representative CM marble samples.

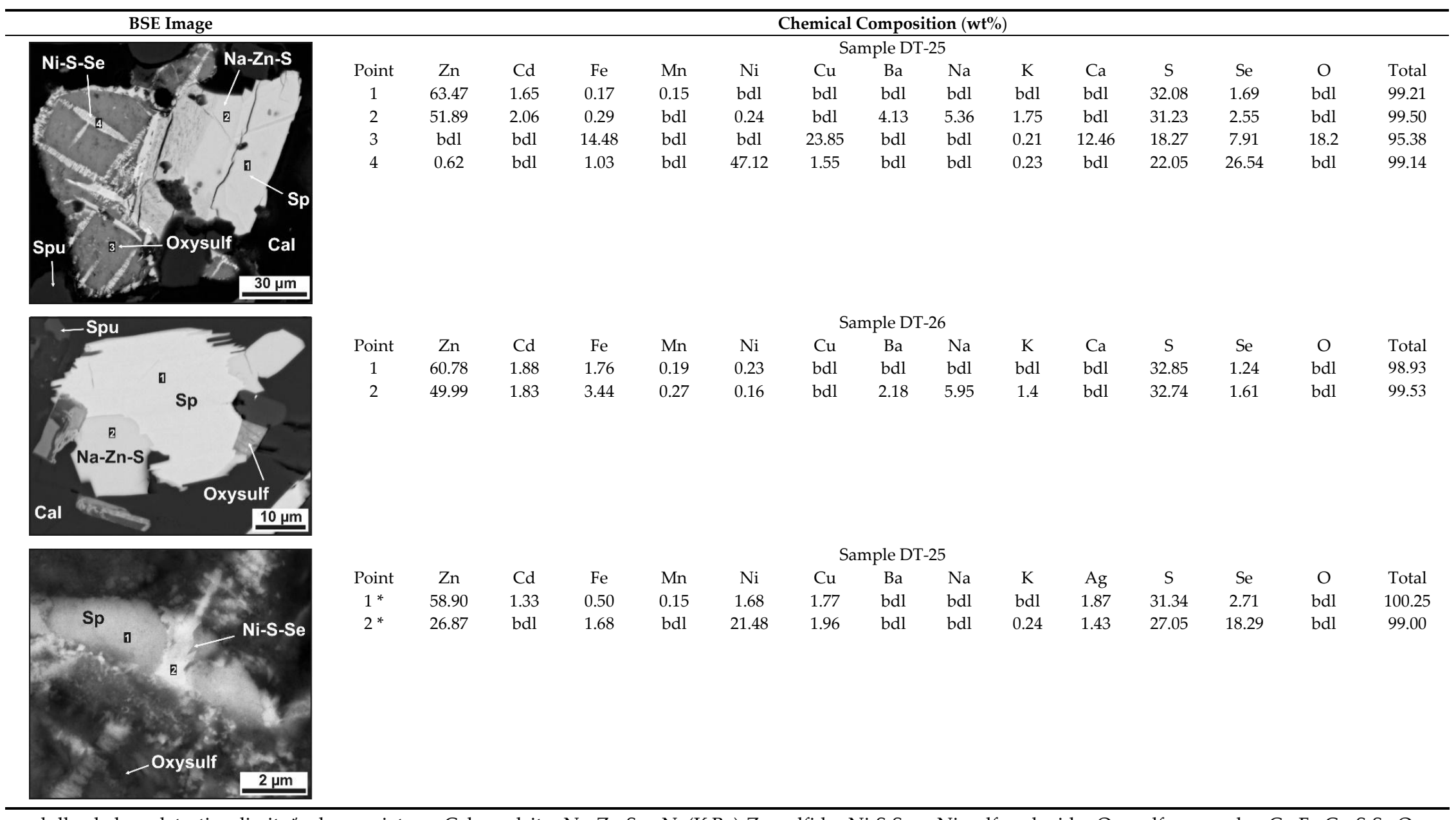

bdl = below detection limit. ${ }^{*}$ phase mixture. $\mathrm{Cal}=$ calcite, $\mathrm{Na}-\mathrm{Zn}-\mathrm{S}=\mathrm{Na}(\mathrm{K}, \mathrm{Ba})-\mathrm{Zn}$ sulfide, Ni-S-Se $=\mathrm{Ni}$ sulfo-selenide, Oxysulf $=$ complex Ca-Fe-Cu-S-Se-O compounds (probably oxysulfides), $\mathrm{Sp}=$ sphalerite, $\mathrm{Spu}=$ spurrite. 
Table 7. BSE images and EDS compositions (wt $\%$ ) of $\mathrm{Zn-}, \mathrm{Cd}$-sulfides, and Ag-, Cd-selenides from the sample DT-25.

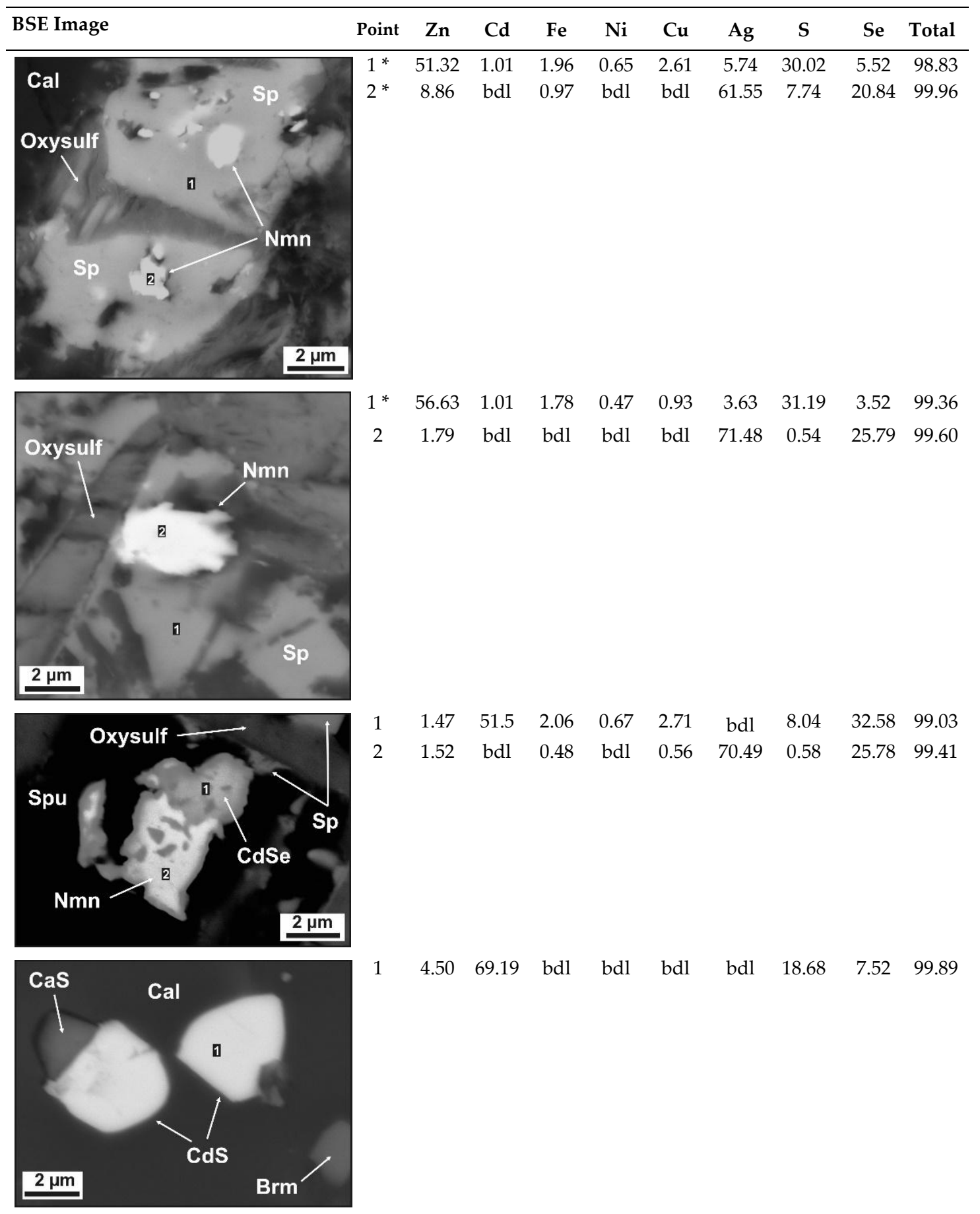

bdl $=$ below detection limit. ${ }^{*}$ phase mixture. $\mathrm{Brm}=$ brownmillerite, $\mathrm{Cal}=$ calcite, $\mathrm{CaS}=$ oldhamite, $\mathrm{CdSe}=$ cadmoselite, $\mathrm{CdS}=$ greenockite, $\mathrm{Nmn}=$ naumannite, Oxysulf $=$ complex $\mathrm{Ca}-\mathrm{Fe}-\mathrm{Cu}-\mathrm{S}-\mathrm{Se}-\mathrm{O}$ compound (probably oxysulfide), $\mathrm{Sp}=$ sphalerite, $\mathrm{Spu}=$ spurrite. 
Table 8. BSE images and EDS composition ( $w \mathrm{t} \%$ ) of oxide accessories associated with sphalerite in the representative $\mathrm{CM}$ marble samples.

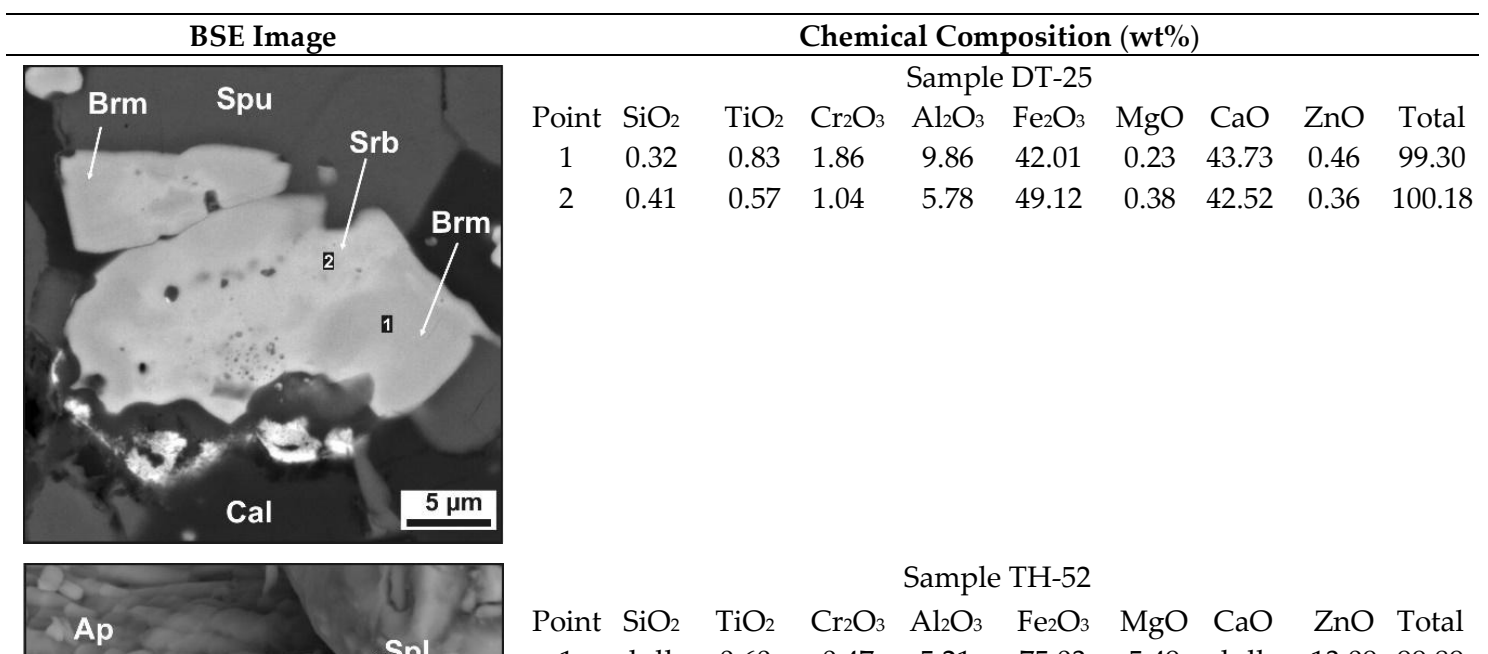

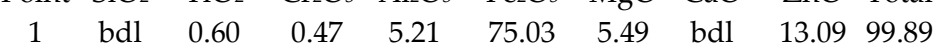

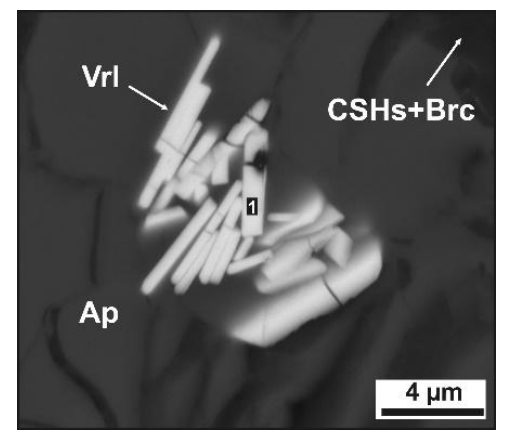

Sample DT-25

Point $\mathrm{SiO}_{2} \quad \mathrm{CaO} \quad \mathrm{MgO} \quad \mathrm{UO}_{3}$ Total

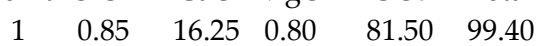

$\mathrm{bdl}=$ below detection limit. $\mathrm{Ap}=$ fluorapatite, $\mathrm{Brc}=$ brucite, $\mathrm{Brm}=$ brownmillerite, $\mathrm{Cal}=$ calcite, $\mathrm{CSH}$ $=$ calcium silicate hydrates, $\mathrm{Spl}=$ spinel-group mineral, $\mathrm{Srb}=$ srebrodolskite, $\mathrm{Vrl}=$ vorlanite .

The DT marbles are fresh and contain intact spurrite and brownmillerite, sporadic Ca silicate hydrates (CSHs), trace amounts of flourmayenite partially converted into chlormayenite $\left(\mathrm{Ca}_{12} \mathrm{Al}_{14} \mathrm{O}_{32}\left[\square_{4} \mathrm{Cl}_{2}\right]\right)$, as well as lime and oldhamite, which survived although being prone to hydration. The $\mathrm{TH}$ marble samples contain $\mathrm{CSHs}$, gypsum, portlandite, $\mathrm{U}$ micas, and hydrated Si-bearing $\mathrm{CaO}-\mathrm{UO}_{3}$ compounds after primary anhydrous $\mathrm{Ca}-\mathrm{U}(\mathrm{VI})$ oxides, chromatite $\left(\mathrm{CaCrO}_{4}\right)$, volkonskoite, and other secondary minerals (Table 4) [14,18,19,23].

\subsection{Combustion Metamorphic Sphalerite: Abundance, Distribution, and Chemistry}

Sphalerite from the Tulul al Hammam CM marbles occurs as compact clusters of abundant coarse grains in DT samples with 1090-1660 ppm Zn and 39-52.7 ppm Cd ( $\mathrm{Zn} / \mathrm{Cd}=27.1-36.9)$. In TH samples with 231 to $470 \mathrm{ppm} \mathrm{Zn}$ and 3 to $75 \mathrm{ppm} \mathrm{Cd}(\mathrm{Zn} / \mathrm{Cd}=4.4-77)$, only sporadic fine grains of $\mathrm{Zn}$ and $\mathrm{Cd}$ sulfides were found. The main impurities in the $\mathrm{CM}$ sphalerite $(\mathrm{Cd}, \mathrm{Se}$, and $\mathrm{Fe})$ reach $\mathrm{wt} \%$ levels (Table 9). The sphalerite grains are free from compositional zoning (Figures 7 and 9), and thus are advantageous for LA-ICPMS analyses. 
Table 9. Selected typical compositions of sphalerite from CM marbles, Tulul al Hammam area (EDS and EPMA data, $w t \%)$.

\begin{tabular}{|c|c|c|c|c|c|c|c|c|c|c|c|c|c|}
\hline \multirow{2}{*}{ Sample } & \multicolumn{4}{|c|}{ DT-20 } & \multicolumn{3}{|c|}{$\begin{array}{l}\text { DT-25 } \\
\end{array}$} & \multicolumn{3}{|c|}{ DT-26 } & \multicolumn{2}{|c|}{ TH-11 } & \multirow{2}{*}{$\begin{array}{c}\text { TH-74 } \\
13\end{array}$} \\
\hline & 1 & 2 & 3 & 4 & 5 & 6 & 7 & 8 & 9 & 10 & 11 & 12 & \\
\hline $\mathrm{Zn}$ & 63.14 & 63.99 & 63.97 & 64.11 & 60.23 & 64.76 & 59.80 & 61.43 & 63.02 & 61.27 & 65.21 & 64.81 & 53.71 \\
\hline $\mathrm{Cd}$ & 1.93 & 1.93 & 2.03 & 1.91 & 1.32 & 1.67 & 3.14 & 1.15 & 1.70 & 3.53 & 1.07 & 1.55 & 14.07 \\
\hline $\mathrm{Fe}$ & 1.16 & 1.41 & 1.39 & 1.32 & 0.85 & 0.17 & 1.15 & 3.48 & 1.78 & 2.03 & 0.40 & 0.44 & 1.56 \\
\hline $\mathrm{Mn}$ & 0.11 & $<0.10$ & $<0.10$ & $<0.10$ & $<0.10$ & $<0.10$ & $<0.10$ & 0.23 & 0.19 & 0.17 & 0.19 & $<0.10$ & 0.56 \\
\hline $\mathrm{Ni}^{*}$ & $<0.10$ & $<0.10$ & $<0.10$ & $<0.10$ & 1.11 & $<0.10$ & 0.35 & $<0.10$ & 0.23 & $<0.10$ & $<0.10$ & $<0.10$ & $<0.10$ \\
\hline $\mathrm{Cu}^{*}$ & $<0.15$ & $<0.15$ & $<0.15$ & $<0.15$ & 0.93 & $<0.15$ & 1.04 & $<0.15$ & $<0.15$ & $<0.15$ & $<0.15$ & $<0.15$ & $<0.15$ \\
\hline $\mathrm{S}$ & 31.91 & 32.78 & 32.52 & 31.38 & 29.53 & 32.38 & 29.50 & 32.50 & 32.44 & 32.28 & 32.30 & 32.07 & 30.03 \\
\hline Se & 0.75 & 0.32 & 0.30 & 0.57 & 5.10 & 1.71 & 4.54 & 1.37 & 1.05 & 0.87 & 0.96 & 1.29 & 0.47 \\
\hline Total & 99.01 & 100.43 & 100.20 & 99.29 & 99.07 & 100.69 & 99.53 & 100.16 & 100.43 & 100.14 & 100.12 & 100.16 & 100.40 \\
\hline \multicolumn{14}{|c|}{ Formula based on $\sum S+\mathrm{Se}=1$} \\
\hline $\mathrm{Zn}$ & 0.961 & 0.954 & 0.961 & 0.960 & 0.935 & 0.970 & 0.936 & 0.921 & 0.940 & 0.921 & 0.978 & 0.975 & 0.851 \\
\hline $\mathrm{Cd}$ & 0.017 & 0.017 & 0.018 & 0.017 & 0.012 & 0.014 & 0.029 & 0.010 & 0.015 & 0.031 & 0.009 & 0.014 & 0.130 \\
\hline $\mathrm{Fe}$ & 0.021 & 0.025 & 0.024 & 0.022 & 0.015 & 0.003 & 0.021 & 0.060 & 0.031 & 0.036 & 0.007 & 0.008 & 0.029 \\
\hline $\mathrm{Mn}$ & 0.002 & 0.000 & 0.000 & 0.000 & 0.000 & 0.000 & 0.000 & 0.004 & 0.003 & 0.003 & 0.003 & 0.000 & 0.010 \\
\hline $\mathrm{Ni}^{*}$ & 0.000 & 0.000 & 0.000 & 0.000 & 0.019 & 0.000 & 0.006 & 0.000 & 0.004 & 0.000 & 0.000 & 0.000 & 0.000 \\
\hline $\mathrm{Cu}$ * & 0.000 & 0.000 & 0.000 & 0.000 & 0.015 & 0.000 & 0.017 & 0.000 & 0.000 & 0.000 & 0.000 & 0.000 & 0.000 \\
\hline S & 0.991 & 0.996 & 0.996 & 0.993 & 0.935 & 0.979 & 0.941 & 0.983 & 0.987 & 0.989 & 0.988 & 0.984 & 0.994 \\
\hline Se & 0.009 & 0.004 & 0.004 & 0.007 & 0.065 & 0.021 & 0.059 & 0.017 & 0.013 & 0.011 & 0.012 & 0.016 & 0.006 \\
\hline$\sum \mathrm{M}$ & 1.001 & 0.995 & 1.003 & 0.999 & 0.996 & 0.988 & 1.009 & 0.996 & 0.994 & 0.990 & 0.998 & 0.997 & 1.020 \\
\hline
\end{tabular}
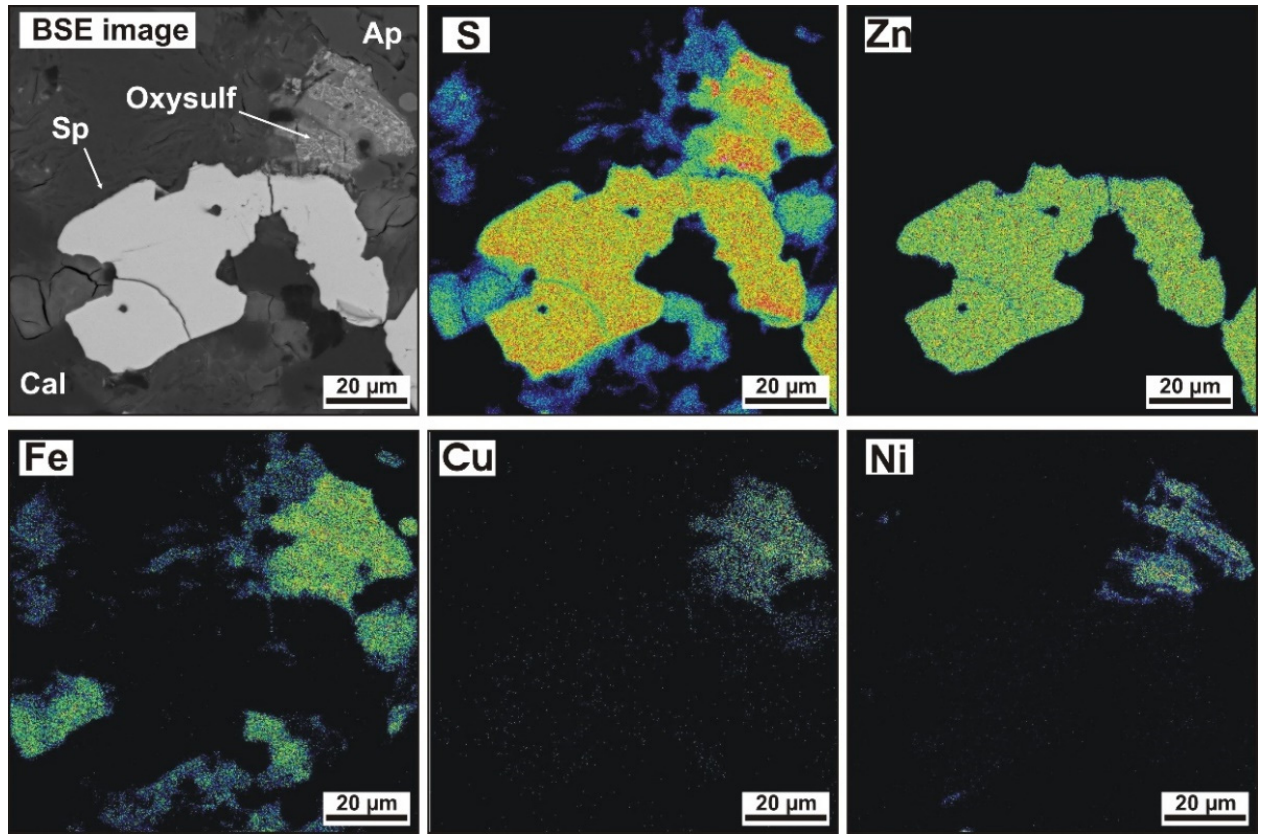

Figure 9. BSE image and elemental maps $(\mathrm{S}, \mathrm{Zn}, \mathrm{Fe}, \mathrm{Cu}, \mathrm{Ni}$ ) showing the chemical homogeneity of sphalerite from sample DT-25. Ap = fluorapatite, $\mathrm{Cal}=$ calcite, $\mathrm{Oxysulf}=$ complex Ca-Fe-Cu-S-Se-O compounds (oxychalcogenides ?), $\mathrm{Sp}=$ sphalerite.

Cadmium in CM sphalerite is $2.01 \mathrm{wt} \%$ on average ( $X_{a v}$ over $\mathrm{N}=200$ determinations) (Figure 10; Table 10). At least $1 \mathrm{wt} \% \mathrm{Cd}$ is present at every analyzed point, but it rarely exceeds $3 \mathrm{wt} \%$. The $\mathrm{Cd}$ contents are the highest $(14.0 \mathrm{wt} \%)$ in the smallest sphalerite grains from sample $\mathrm{TH}-74$ with the lowest $\mathrm{Zn} / \mathrm{Cd}$ ratio of 4.4. Cd-rich sphalerites from samples TH-74 and DT-25 coexist with sporadic tiny grains of primary or secondary greenockite $\left(\mathrm{Cd}_{0.73-0.99} \mathrm{Zn}_{0.19-0.35} \mathrm{Fe}_{0.02-0.07} \mathrm{~S}_{0.80-0.98} \mathrm{Se}_{0.02-0.20}\right)$ and very rarely with cadmoselite (CdSe) (Tables 7 and 11). The Cd concentrations in CM sphalerites poorly correlate with the contents of other elements. 
Selenium is another ubiquitous impurity in the CM sphalerites. It is uniformly distributed within each grain (Figure 8 ) but varies mostly from $0.3-0.8 \mathrm{wt} \%$ to $1.4 \mathrm{wt} \%$ between grains even in the same thin sections (Tables 9 and 10; Figure 10). The average Se contents are in a range of 0.51-1.12 wt $\%$. Expectedly, the highest Se concentration $\left(X_{a v}=4.27 \mathrm{wt} \% ; X_{\max }=6.68 \mathrm{wt} \% \mathrm{Se}\right)$ was obtained for sample DT-25 with 503 ppm of bulk Se. Se-rich sphalerite belongs to assemblages that include Se-rich Ni, Cu, and Ag sulfides, selenides, and oxysulfides (Tables 4 and 7; Figure 8). However, in the same sample Se in coarse sphalerite grains outside selenide accumulations ranges from 1.7 to $4.3 \mathrm{wt} \%$ Se. The Se content is the highest (up to $\left.10.61 \mathrm{wt} \% ; X_{a v}=5.78 \mathrm{wt} \%\right)$ in greenockite and cadmoselite $((\mathrm{Cd}, \mathrm{Zn})(\mathrm{Se}, \mathrm{S}))$.

Iron is in a range of $1.2-2.2 \mathrm{wt} \%\left(X_{a v}=1.46 \mathrm{wt} \% ; \mathrm{N}=185\right)$ in most of the analyzed sphalerite grains (Tables 9 and 10; Figure 10). It is the lowest $(0.31-0.64 \mathrm{wt} \%$ ) in sphalerite from sample TH-11 and the highest in that from DT-26 (up to $3.74 \mathrm{wt} \%$ ).

Consistent SEM-EDS, EPMA, and LA-ICPMS data for the representative sample DT-20 (Tables 3, 9 and 10), as well as elemental mapping (Figures 7 and 9), confirm the uniform distribution of $\mathrm{Cd}$, Se, and Fe in the CM sphalerites. Single-crystal XRD data (see below) prove that these elements are randomly distributed in the cubic $\mathrm{ZnS}$ structure.

Manganese in the analyzed sphalerites is most often below the EDS-SEM detection limit $(<0.1 \mathrm{wt} \%)$. Sporadic spikes of $0.18-0.66 \mathrm{wt} \% \mathrm{Mn}$ may be due to inclusions of secondary Mn (oxy)hydroxides. LA-ICPMS measurements reveal 1440-1780 ppm Mn $\left(X_{a v}=1565\right.$ ppm; $\left.\mathrm{N}=16\right)$ in sphalerite from DT-20 and as low as 355 ppm in DT-25. Generally, the contents of Mn are an order of magnitude higher than those of cobalt and nickel. Co contents reach $172 \mathrm{ppm}\left(X_{a v}=124 \mathrm{ppm}\right.$ in DT-20 and $X_{a v}=66 \mathrm{ppm}$ in DT-25) (Table 3). The average Ni contents in large homogeneous sphalerite crystals are 265 ppm in Ni-rich sample DT-25 and 220 ppm in sample DT-20 free from Ni phases. The absence of spikes supports the idea that high $\mathrm{Ni}$ contents are due to lattice-bound nickel rather than to inclusions of Ni-rich phases. However, the limited number of samples and analyses requires caution in such conclusions.

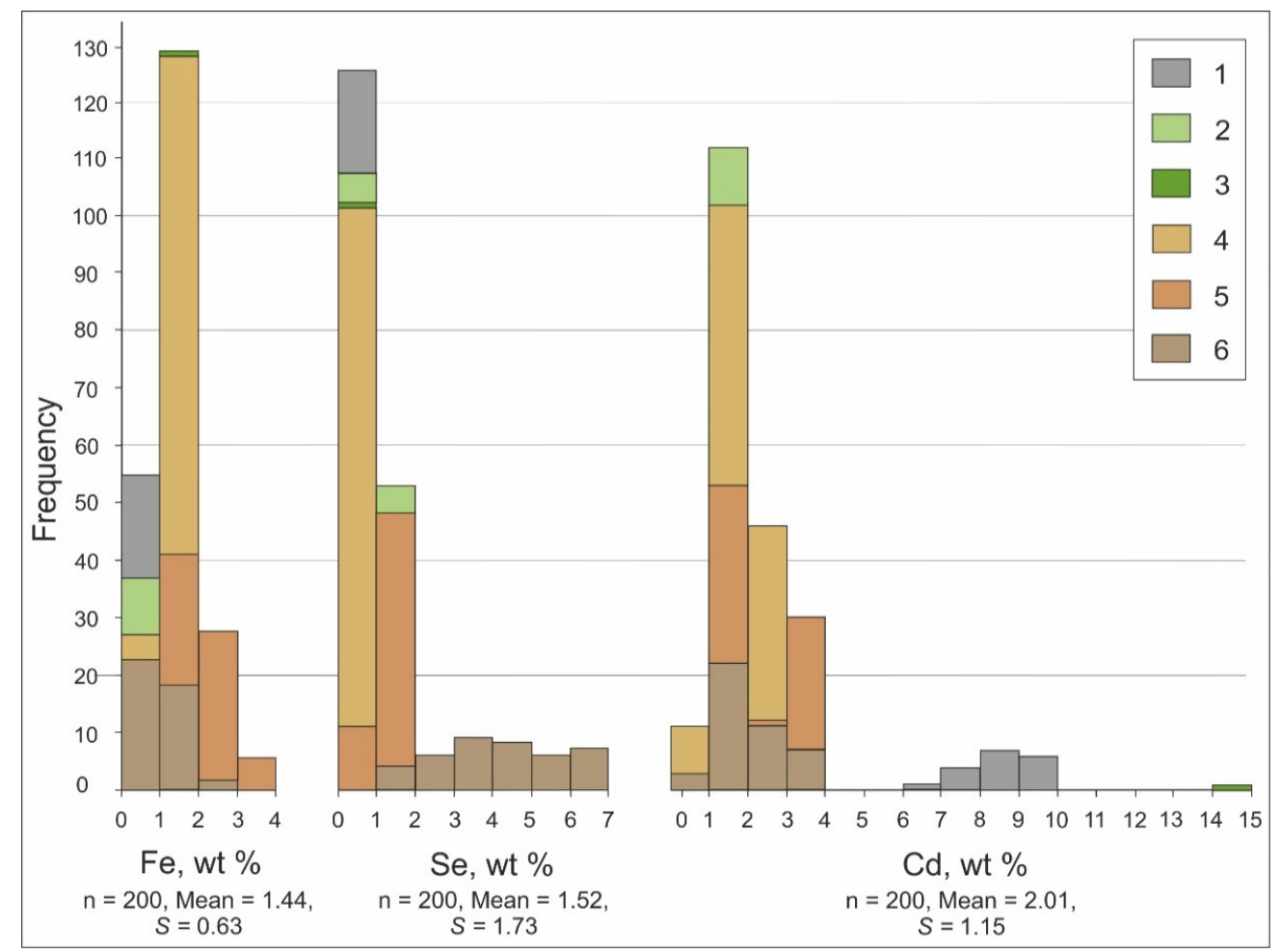

Figure 10. Histograms of $\mathrm{Fe}$, Se, and $\mathrm{Cd}$ in $\mathrm{ZnS}$ from sedimentary (1) and CM (2-6) rocks. Summary of SEM-EDS, EPMA, and LA-ICPMS data. $\mathrm{n}=$ number of analyses, Mean = mean value, $S=$ standard deviation. Statistical data calculated for ZnS from CM rocks. Samples: $1=$ DOS, OSB; $2=$ TH-11; $3=$ TH-74; 4 = DT-20; 5 = DT-26; 6 = DT-25. 
Table 10. Average compositions of sphalerite from CM marbles, Tulul al Hammam area (SEM-EDS and EPMA, wt\%).

\begin{tabular}{|c|c|c|c|c|c|c|c|c|c|c|c|c|c|c|c|c|}
\hline \multirow{2}{*}{ Sample } & \multicolumn{4}{|c|}{ DT-20 (n = 45) } & \multicolumn{4}{|c|}{ DT-25 (n = 21) } & \multicolumn{4}{|c|}{ DT-26 $(n=53)$} & \multicolumn{4}{|c|}{ TH-11 $(n=10)$} \\
\hline & Mean & $S$ & Min & Max & Mean & $S$ & Min & Max & Mean & $S$ & Min & Max & Mean & $S$ & Min & Max \\
\hline $\mathrm{Zn}$ & 63.28 & 0.43 & 62.44 & 63.99 & 61.16 & 1.86 & 58.46 & 64.96 & 61.98 & 0.84 & 60.54 & 64.19 & 64.80 & 0.34 & 64.07 & 65.24 \\
\hline $\mathrm{Cd}$ & 2.02 & 0.28 & 1.63 & 2.64 & 1.84 & 0.64 & 0.95 & 3.14 & 2.21 & 1.06 & 1.04 & 3.73 & 1.35 & 0.17 & 1.07 & 1.55 \\
\hline $\mathrm{Fe}$ & 1.28 & 0.11 & 1.04 & 1.44 & 1.13 & 0.60 & $<0.10$ & 2.15 & 2.22 & 0.54 & 1.55 & 3.71 & 0.45 & 0.09 & 0.32 & 0.66 \\
\hline $\mathrm{Mn}$ & 0.10 & 0.06 & $<0.10$ & 0.16 & $<0.10$ & - & $<0.10$ & $<0.10$ & 0.15 & 0.07 & $<0.10$ & 0.24 & $<0.10$ & - & $<0.10$ & $<0.10$ \\
\hline $\mathrm{Ni}^{*}$ & $<0.10$ & - & $<0.10$ & 0.30 & 0.46 & 0.47 & $<0.10$ & 1.83 & $<0.10$ & - & $<0.10$ & 0.31 & $<0.10$ & - & $<0.10$ & $<0.10$ \\
\hline $\mathrm{Cu}^{*}$ & $<0.15$ & - & $<0.15$ & $<0.15$ & 0.85 & 0.69 & $<0.15$ & 2.70 & $<0.15$ & - & $<0.15$ & $<0.15$ & $<0.15$ & - & $<0.15$ & $<0.15$ \\
\hline $\mathrm{S}$ & 32.56 & 0.33 & 31.91 & 33.10 & 30.10 & 1.30 & 27.86 & 32.47 & 32.45 & 0.25 & 31.83 & 32.99 & 32.15 & 0.19 & 31.78 & 32.41 \\
\hline $\mathrm{Se}$ & 0.51 & 0.13 & 0.29 & 0.75 & 4.27 & 1.67 & 1.39 & 6.68 & 1.12 & 0.13 & 0.77 & 1.37 & 1.05 & 0.14 & 0.87 & 1.29 \\
\hline Total & 99.65 & & & & 99.81 & & & & 100.13 & & & & 99.80 & & & \\
\hline
\end{tabular}

Concentrations of $\mathrm{Co}(<0.10 \mathrm{wt} \%)$ and $\mathrm{Ag}(<0.15 \mathrm{wt} \%)$ are below detection limit. ${ }^{*}$ may be captured from coexisting phases during analysis. $\mathrm{n}=$ number of analyses, Mean $=$ mean value $S=$ standard deviation, Min = minimum value, Max = maximum value.

Table 11. Representative analyses and average composition of greenockite from CM marbles, Tulul al Hammam area (SEM-EDS, wt $\%$ ).

\begin{tabular}{|c|c|c|c|c|c|c|c|c|c|c|c|}
\hline \multirow{2}{*}{ Sample } & \multirow{2}{*}{$\begin{array}{c}\text { TH-74 } \\
1\end{array}$} & \multicolumn{6}{|c|}{ DT-25 } & \multicolumn{4}{|c|}{ DT-25 ( $n=14)$} \\
\hline & & 2 & 3 & 4 & 5 & 6 & 7 & Mean & $S$ & Min & Max \\
\hline $\mathrm{Cd}$ & 55.74 & 75.04 & 69.94 & 69.57 & 64.54 & 63.46 & 63.07 & 68.29 & 4.79 & 63.07 & 75.05 \\
\hline $\mathrm{Zn}$ & 17.93 & 0.69 & 3.89 & 4.34 & 8.19 & 10.83 & 11.24 & 5.96 & 4.16 & 0.60 & 11.24 \\
\hline $\mathrm{S}$ & 24.21 & 18.60 & 18.78 & 18.64 & 17.31 & 22.03 & 21.62 & 19.46 & 1.38 & 17.31 & 22.03 \\
\hline Se & 1.37 & 5.32 & 6.45 & 7.39 & 10.61 & 2.71 & 3.11 & 5.78 & 2.07 & 2.71 & 10.61 \\
\hline Total & 99.25 & 99.65 & 99.06 & 99.94 & 100.65 & 99.03 & 99.04 & 99.49 & & & \\
\hline \multicolumn{12}{|c|}{ Formula based on one $\mathrm{S}$ atom } \\
\hline $\mathrm{Cd}$ & 0.642 & 1.031 & 0.932 & 0.917 & 0.852 & 0.783 & 0.786 & 0.893 & & & \\
\hline $\mathrm{Zn}$ & 0.355 & 0.016 & 0.089 & 0.098 & 0.186 & 0.230 & 0.241 & 0.134 & & & \\
\hline S & 0.978 & 0.896 & 0.878 & 0.861 & 0.801 & 0.952 & 0.945 & 0.892 & & & \\
\hline Se & 0.022 & 0.104 & 0.122 & 0.139 & 0.199 & 0.048 & 0.055 & 0.108 & & & \\
\hline$\sum \mathrm{Cd}+\mathrm{Zn}$ & 0.997 & 1.047 & 1.021 & 1.015 & 1.037 & 1.012 & 1.027 & 1.028 & & & \\
\hline
\end{tabular}

$\mathrm{Mn}, \mathrm{Fe}, \mathrm{Ni}(<0.10 \mathrm{wt} \%), \mathrm{Cu}(<0.15 \mathrm{wt} \%)$ are below detection limit. $\mathrm{n}=$ number of analyses, Mean = mean value, $S=$ standard deviation, Min $=$ minimum value, Max $=$ maximum value. 
The composition of smaller sphalerite grains that coexist with intricately intergrown $\mathrm{Ni}, \mathrm{Cu}$, and $\mathrm{Ag}$ sulfides and selenides was resolvable only by SEM-EDS. Such grains commonly contain $0.3-3.0 \mathrm{wt} \%$ $\mathrm{Ni}$ and $0.4-2.7 \mathrm{wt} \% \mathrm{Cu}$. A few grains with naumannite $\left(\mathrm{Ag}_{2} \mathrm{Se}\right)$ inclusions also bear $1.3-3.0 \mathrm{wt} \% \mathrm{Ag}$. The element contents are unevenly distributed and have a high standard deviation commensurate with average concentrations, possibly, because of submicrometer inclusions of the respective phases irresolvable even in BSE images (Tables 7 and 9).

Mercury concentrations in the CM sphalerites (Table 3) are relatively high (132 and 226 ppm), being of the same order of magnitude as in the authigenic $\mathrm{ZnS}$ precursors (345 ppm). Copper varies from 3.7 to $33.4 \mathrm{ppm}\left(X_{a v}=11.1 \mathrm{ppm}\right)$.

The contents of other elements reveled by LA-ICPMS in different crystals of the representative marble sample DT-20 are 0.3-1.37 ppm Ga $\left(X_{a v}=0.45 \mathrm{ppm}\right), 0.15-0.62 \mathrm{ppm} \mathrm{Ag}\left(X_{a v}=0.42 \mathrm{ppm}\right)$, 4.11-4.9 2 ppm In $\left(X_{a v}=4.51 \mathrm{ppm}\right), 0.83-1.53 \mathrm{ppm}$ Sn $\left(X_{a v}=1.10 \mathrm{ppm}\right)$, and 0.18-6.33 ppm Sb $\left(X_{a v}=1.19 \mathrm{ppm}\right)($ Table 3$)$. The $\mathrm{Cu}, \mathrm{Ag}, \mathrm{Sn}$, and $\mathrm{Sb}$ concentrations in a few large sphalerites from sample DT-25 with higher TE loading are within the same ranges, while In and Ga are slightly higher (2.44 and 7.9 ppm, respectively). Sphalerites from both samples are depleted in $\mathrm{Ge}, \mathrm{As}, \mathrm{Mo}, \mathrm{Te}, \mathrm{Tl}, \mathrm{Bi}, \mathrm{Pb}, \mathrm{Au}$, and $\mathrm{Pt}$, which are below the detection limits. SEM-EDS and EPMA data for accessories in the Jordanian $\mathrm{CM}$ marbles revealed $\mathrm{Cd}$ and Se enrichment and the presence of Fe in sphalerites. Other important impurities have never been reliably detected by EPMA as lattice-bound species. Spikes of $\mathrm{Cu}, \mathrm{Ni}$, and/or Ag are most likely associated with submicron inclusions. On the other hand, analysis of sphalerite has systematically shown deficient totals (up to $1 \mathrm{wt} \%$ ), especially in high-quality homogeneous sphalerite crystals from DT-20 free from micrometer inclusions (Figure 5C-F). We tentatively attribute the deficit to some impurities that may have concentrations below the EPMA detection limits. However, the exhaustive trace-element determinations by LA-ICPMS (Table 3) fail to account for the whole difference: The TE total (without $\mathrm{Cd}$, Se and $\mathrm{Fe}$ ) is no higher than $0.2 \mathrm{wt} \%$. Otherwise, the deficit of totals may be due to the oxygen detected in some crystals by SEM-EDS in amounts of 0.5-1.0 wt\%, but these results can only be used as diagnostic because of a high detection limit $(0.4 \mathrm{wt} \%)$ and a very large relative error ( $40 \%$ at low contents). The $\mathrm{S}^{2-} \rightarrow \mathrm{O}^{2-}$ substitution range was constrained by single-crystal XRD.

\subsection{X-Ray Diffraction and Crystal Structure}

Crystal structure was determined in a pure transparent sphalerite crystal $(\sim 160 \times 80 \times 60 \mu \mathrm{m})$, with a $0.65 \mathrm{wt} \%$ deficit of totals, which was extracted from sample DT-20 (Table 9 , analysis 4 ). The mineral is cubic $\mathrm{ZnS}$, with cell parameters of $a=5.40852(12) \AA, V=158.211(6) \AA^{3}$, and $Z=4$. Systematic absence of reflections is consistent with the space-group $F \overline{4} 3 m$. The unit-cell size of $(\mathrm{Zn}, \mathrm{Cd}, \mathrm{Fe})(\mathrm{S}, \mathrm{Se}, \mathrm{O})$ approaches that of pure $\mathrm{ZnS}(a=5.4094 \AA$ [56]). The crystal structure was solved and refined on the basis of $F^{2}$ using the SHELX software (Version 2018/3, Göttingen University, Göttingen, Germany) [57]. Like the pure endmember $\mathrm{ZnS}_{\mathrm{cub}}$, natural sphalerite has a zinc blende-type structure, without any discernible ordering of cations and/or anions and/or superstructure. Single-crystal XRD failed to reveal any reflections that would represent inclusions or domains of extraneous (e.g., O-bearing) phases, such as $\mathrm{ZnO}$ or $\mathrm{Zn}$ sulfates.

The structure (Table 12) was refined assuming $\mathrm{Zn}^{2+}, \mathrm{Cd}^{2+}$, and $\mathrm{Fe}^{2+} \mathrm{M}$-site occupancy proceeding from chemical analyses. The share of Se in the anionic occupancy was inferred from EPMA and SEM-EDS data. The $S$ and $\mathrm{O}$ occupancies of the anionic position were refined assuming its full population.

The resulting formula of the sphalerite is $\mathrm{Zn}_{0.96} \mathrm{Cd}_{0.02} \mathrm{Fe}_{0.02}\left(\mathrm{~S}_{0.946(8)} \mathrm{O}_{0.046(8)} \mathrm{Se}_{0.0074}\right)_{\sum 1.00}$. The XRD analysis detected an excess of oxygen atoms relative to the composition determined by chemical analyses (given that oxygen was assumed to account for the deficit of totals) and the corresponding deficit of sulfur, but the difference is about the error in the respective element contents.

The analyzed sphalerite has a smaller unit-cell size ( $a$ parameter) than pure $\mathrm{ZnS}_{\mathrm{cub}}$. As Skinner and Barton (1960) [58] found out, the $a$ parameter decreases as oxygen substitutes for sulfur: to $5.4081 \AA$ at $0.4 \mathrm{~mol} \% \mathrm{ZnO}$ and to $5.4065-5.4070 \AA$ at $0.8 \mathrm{~mol} \% \mathrm{ZnO}$. The sample we analyzed, with $\sim 0.5 \mathrm{~mol} \%$ 
$\mathrm{ZnO}$, has only a slightly greater unit cell of 5.40852(12) $\AA$, due to the complex composition of the natural crystal. The presence of $\mathrm{Cd}, \mathrm{Fe}$, and Se impurities in the structure jointly leads to a minor unit cell increase. Among all impurities present in the sample, $\mathrm{Cd}$ causes the strongest influence on the cell size while the effect of Fe is less significant [59-61].

The valence of cations for $(\mathrm{Zn}, \mathrm{Cd}, \mathrm{Fe})(\mathrm{S}, \mathrm{O}, \mathrm{Se})$ calculated using the bond valence sum (BVS) formula is +2.04 and approaches that for pure sphalerite $(+2.02)$, with the $v_{i j}=\exp \left[\left(R_{i j}-d_{i j}\right) / 0.37\right]$ contribution of each bond with the distance $d_{i j}$ to the valence, where $R_{i j}$ as an empirical parameter and the total valence $V_{i}$ of the atom $i$ is $V_{i}=\sum_{j} v_{i j}$ [62]).

Thus, the natural crystal of the complex composition $(\mathrm{Zn}, \mathrm{Cd}, \mathrm{Fe})(\mathrm{S}, \mathrm{O}, \mathrm{Se})$ is isostructural to sphalerite and has a disordered distribution of all elements according to the respective $\mathrm{ZnS}_{\text {cub }}$ positions.

Table 12. Crystal parameters, data collection and structure refinement details for Fe-, Cd-, Se-, O-bearing sphalerite.

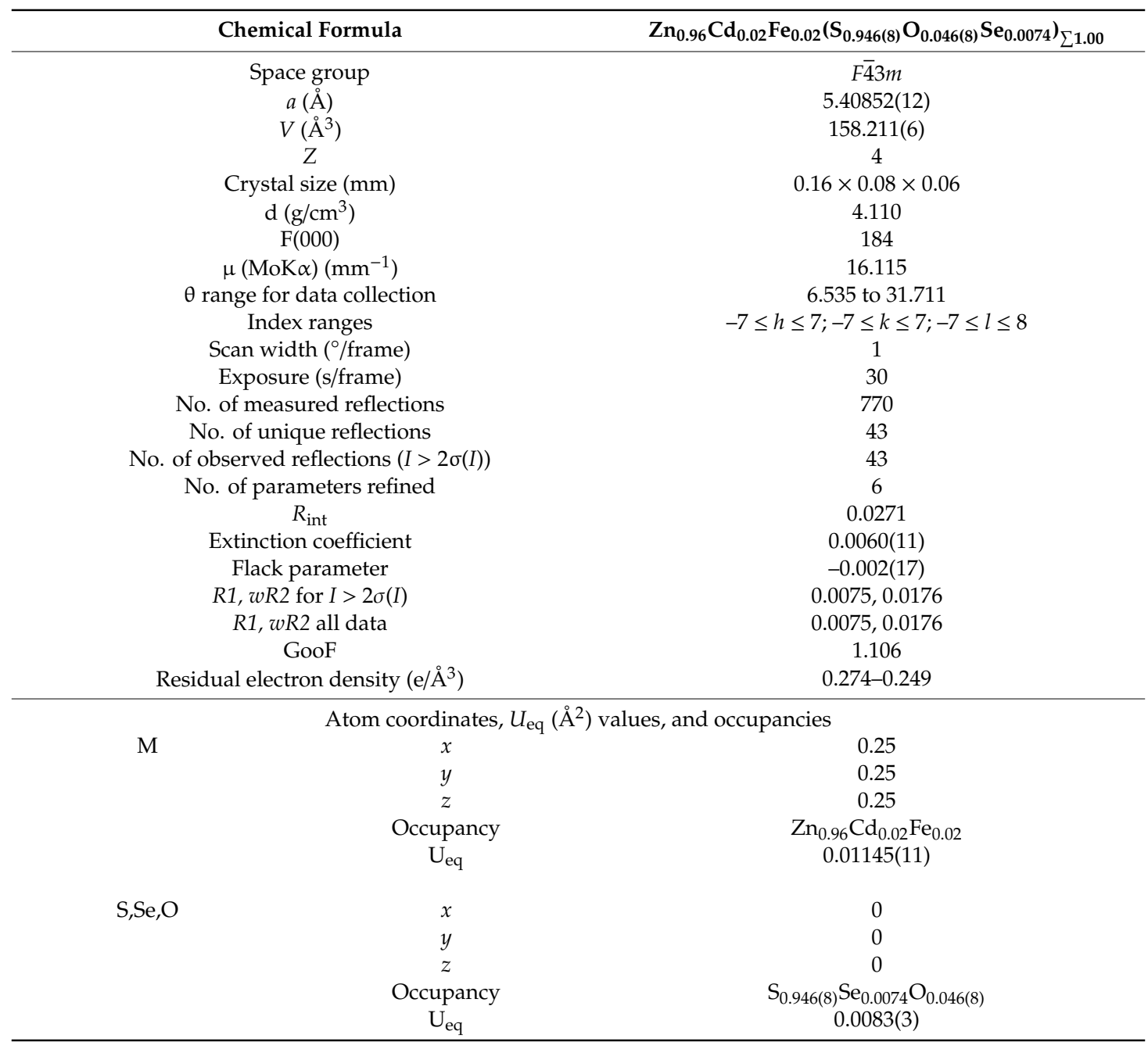

\subsection{Raman Spectroscopy}

\subsubsection{First-Order Raman Spectrum}

The recorded Raman spectra of the Tulul Al Hamman CM sphalerites are generally similar to those reported in the literature, including the RRUFF database (an integrated database of Raman spectra, X-ray diffraction and chemistry data for minerals) $[63,64]$ (Figure 11). Factor-group analysis of vibration modes of the cubic $\bar{F} \overline{4} 3 m\left(T_{d}^{2}\right)$ cell of $\mathrm{ZnS}$ cub (zinc blende-type of structure) predicts two (acoustic and optical) triple-degenerated modes, which both have the same symmetry $F_{2}$ at 
the central point $\Gamma$ of the Brillouin zone. Namely, the $\bar{F} \overline{4} 3 m$ structure at the ambient conditions has total irreducible representation at $k=0: \Gamma=2 F_{2}$. According to our LADY (LAttice Dynamics) calculations of lattice dynamics, only one triple-degenerated optical mode $F_{2}$ is active in the first-order Raman spectrum: the one that represents the stretching vibrations of the $(\mathrm{Zn}, \mathrm{Fe}, \mathrm{Cd})-\mathrm{S}$ bond in corner-connected tetrahedra $(\mathrm{Zn}, \mathrm{Fe}, \mathrm{Cd}) \mathrm{S}_{4}$. This mode splits into double-degenerated transverse (TO) and longitudinal (LO) components upon the interaction of electron states and atomic vibrations with the electromagnetic field of photons.

The first-order Raman spectrum of sphalerite (Figure 12) exhibits one very strong LO band at $344 \mathrm{~cm}^{-1}$ and one weak TO band at $276 \mathrm{~cm}^{-1}$, which agree with the lattice dynamics modeling. However, it additionally contains two very strong bands at 299 and $331 \mathrm{~cm}^{-1}$, as well as a satellite band of moderate intensity at $309 \mathrm{~cm}^{-1}$ with a shoulder of the $299 \mathrm{~cm}^{-1}$ band. All these bands are interpreted in Table 13.

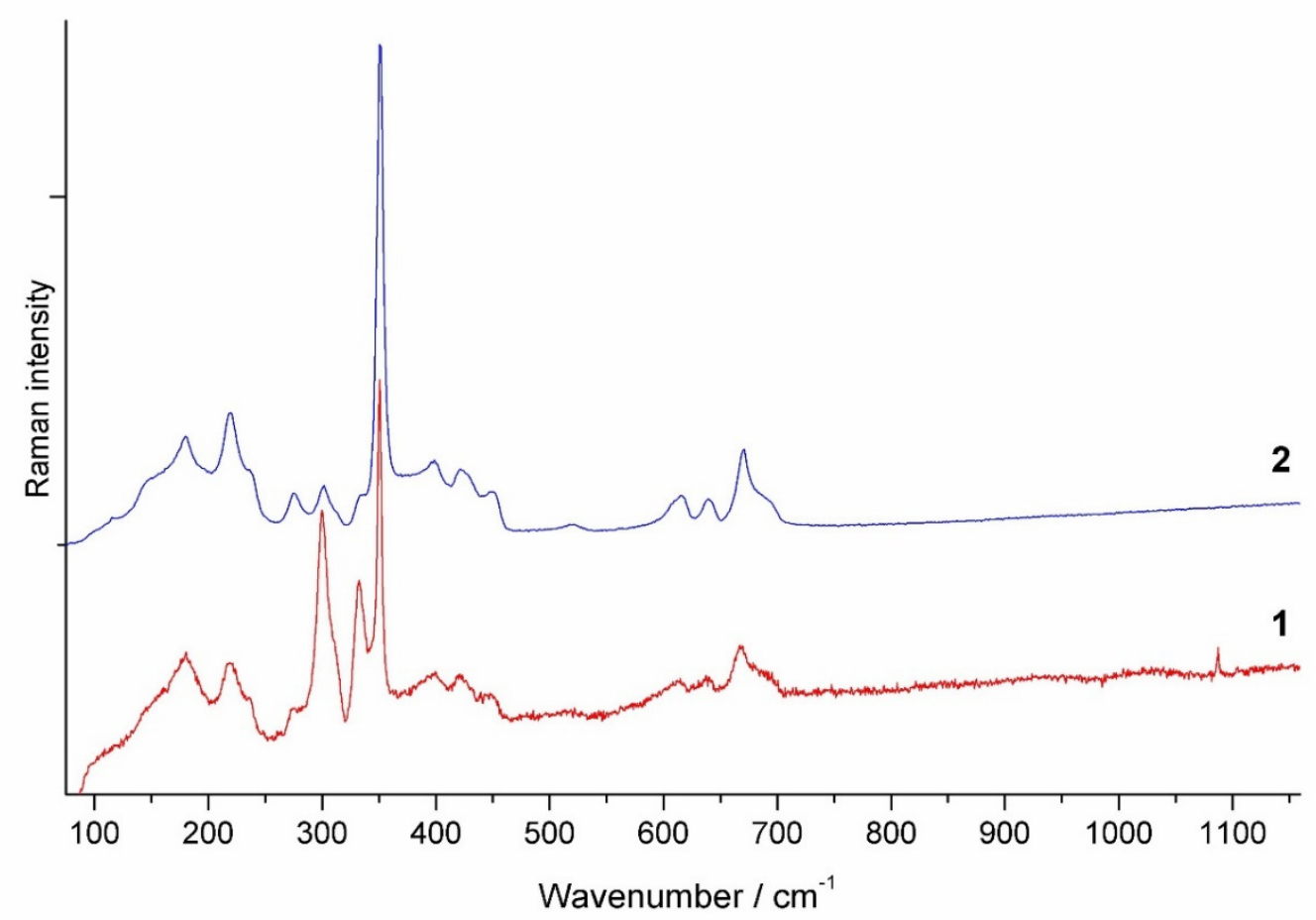

Figure 11. Raman spectra of natural CM sphalerite from sample DT-20 (1) compared with sphalerite from Arizona [RRUFF base] (2).

The spatial micro-Raman analysis of the sample applied to many points of sphalerite grains proves the homogeneity of the solid solution. It revealed no inclusions of extraneous phases (oxides, sulfates, sulfites etc.), layers or films of Zn oxide, or other O- and Zn-bearing compounds, e.g., no bands at 437 and $524 \mathrm{~cm}^{-1}$ (the latter with a shoulder at $562 \mathrm{~cm}^{-1}$ ) corresponding to hexagonal zincite $\mathrm{ZnO}$.

\subsubsection{Second-Order Raman Spectrum}

The second-order Raman spectrum is more intense than the first-order one and more intense than the spectrum of crystalline Si. It contains peaks that represent the density of phonon states at critical points of the Brillouin zone. The bands observed in the spectrum are assigned to combinations of the critical points in cubic ZnS (Table 14). The obtained Raman spectrum of CM sphalerite is similar to that of synthetic sphalerite reported by [63], with similar or the same frequencies (Table 14). 


\subsubsection{Resonant Raman Scattering}

Raman spectra collected at excitation wavelengths of 325 (green line, a) and $325 \mathrm{~nm}$ (UV line, b) show resonance at UV excitation, with overtones of the basic tone (LO mode $348 \mathrm{~cm}^{-1}$ ) (Figure 12; Table 13). The wavenumber of strong bands $(n \mathrm{LO})$ in the resonant spectra of the analyzed sphalerite depends linearly on the number $n$ of repeated basic tone reflections, which appears in the spectra of the $(\mathrm{Zn}, \mathrm{Cd}, \mathrm{Fe})(\mathrm{S}, \mathrm{O}, \mathrm{Se})_{\text {cub }}$ crystal (Figure 13).

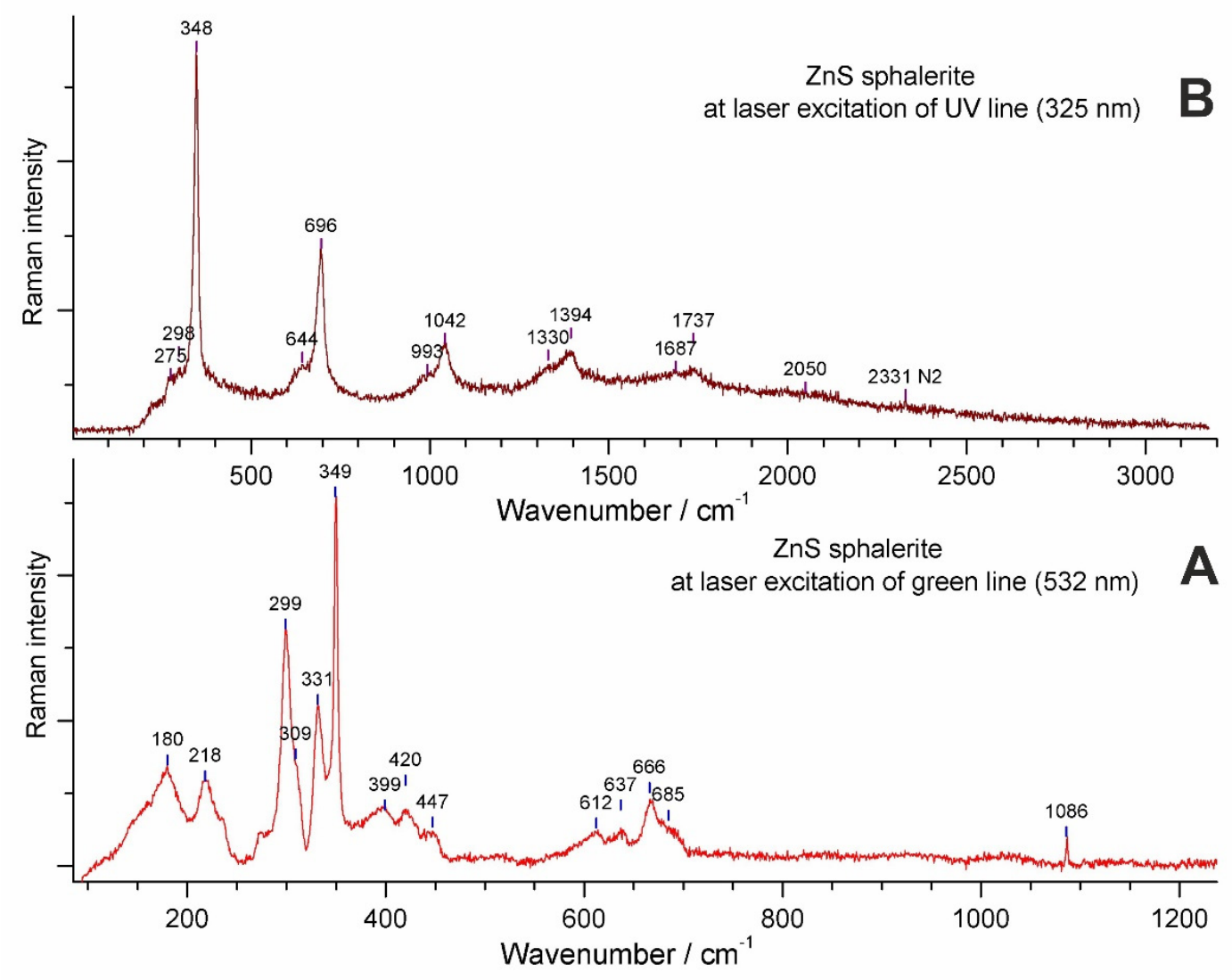

Figure 12. Raman spectra of the Tulul al Hammam CM sphalerite at 532 (A) and $325 \mathrm{~nm}$ (B) excitation. See resonant light scattering in the UV excitation pattern, with several overtones (Table 13) of the main tone (LO mode $348 \mathrm{~cm}^{-1}$ ). The spectras of record a calcite microinclusion (band $1086 \mathrm{~cm}^{-1}$ ) in panels (A) and air N2 (sharp band at $2331 \mathrm{~cm}^{-1}$ ) in (B).

Table 13. Features of first-order Raman spectra of natural CM sphalerite and their interpretation.

\begin{tabular}{|c|c|c|c|c|c|}
\hline $\begin{array}{c}\text { This Study } \\
\lambda \mathrm{L}=532 \mathrm{~nm} \\
v\left(\mathrm{~cm}^{-1}\right)\end{array}$ & $\begin{array}{c}\text { This Study } \\
\lambda \mathrm{L}=325 \mathrm{~nm} \\
v\left(\mathrm{~cm}^{-1}\right)\end{array}$ & $\begin{array}{c}\text { Data from }[63] \\
300 \mathrm{~K}, \lambda \mathrm{L}=514 \mathrm{~nm} \\
v\left(\mathrm{~cm}^{-1}\right)\end{array}$ & $\begin{array}{c}\text { Data from }[64] \\
\lambda \mathrm{L}=514 \mathrm{~nm} \\
v\left(\mathrm{~cm}^{-1}\right)\end{array}$ & $\begin{array}{c}\text { Data from }[64] \\
\lambda \mathrm{L}=325 \mathrm{~nm} \\
v\left(\mathrm{~cm}^{-1}\right)\end{array}$ & $\begin{array}{c}\text { Interpretation } \\
\text { of Bands }\end{array}$ \\
\hline 276 & 275 & 275 & 277 & 277 & TO \\
\hline 299 & 298 & & & & $\mathrm{LO}(\mathrm{X} 1)$ \\
\hline 309 & & & & & $\mathrm{LO}(\mathrm{X} 2)$ \\
\hline 331 & & & & & $\mathrm{LO}(\mathrm{X3})$ \\
\hline \multirow[t]{5}{*}{349} & 348 & 350 & 348 & 348 & LO \\
\hline & 696 & & & 697 & $2 \mathrm{LO}$ \\
\hline & 1042 & & & 1045 & $3 \mathrm{LO}$ \\
\hline & 1394 & & & & $4 \mathrm{LO}$ \\
\hline & 1737 & & & & $5 \mathrm{LO}$ \\
\hline
\end{tabular}


Table 14. Features of second-order Raman spectra of $\mathrm{ZnS}_{\mathrm{cub}}$ (sphalerite) and their interpretation.

\begin{tabular}{|c|c|c|}
\hline $\begin{array}{c}\text { This Study } \\
v\left(\mathrm{~cm}^{-1}\right)\end{array}$ & $\begin{array}{c}\text { Data from [63] } \\
v\left(\mathrm{~cm}^{-1}\right)\end{array}$ & Interpretation of Bands \\
\hline 147 & 142 & $2 \mathrm{TA}_{\mathrm{L}}$ \\
\hline \multirow[t]{2}{*}{180} & 180 & $2 \mathrm{TA}_{\mathrm{X}}$ \\
\hline & 198 & $\left(\mathrm{TO}-\mathrm{TA}_{\mathrm{u}}\right)_{\Sigma}$ \\
\hline 218 & 219 & $(\mathrm{TO}-\mathrm{TA})_{\mathrm{X}}$ \\
\hline \multirow[t]{2}{*}{234} & 239 & $2 \mathrm{TA}_{\Sigma}$ \\
\hline & 304 & $\left(\mathrm{LA}+\mathrm{TA}_{1}\right)_{\mathrm{W}}$ \\
\hline \multirow[t]{3}{*}{309} & 312 & $\left(\mathrm{LA}+\mathrm{TA}_{\mathrm{u}}\right)_{\mathrm{W}, \Sigma}$ \\
\hline & 336 & Not assigned \\
\hline & 346 & Not assigned \\
\hline 385 & 380 & $2 \mathrm{LA} \mathrm{A}_{\Sigma}$ \\
\hline 399 & 397 & $(\mathrm{TO}+\mathrm{TA})_{\mathrm{X}}$ \\
\hline 420 & 420 & $(\mathrm{LO}+\mathrm{TA})_{X}$ \\
\hline \multirow[t]{2}{*}{447} & 450 & $(\mathrm{LO}+\mathrm{TA})_{\mathrm{L}}$ \\
\hline & 483 & $\left(\mathrm{TO}_{1}+\mathrm{LA}\right)_{\Sigma}$ \\
\hline \multirow[t]{2}{*}{515} & 517 & $(\mathrm{LO}+\mathrm{LA})_{\Sigma}$ \\
\hline & 607 & $2 \mathrm{TO}_{\mathrm{W}}$ \\
\hline 612 & 615 & $2 \mathrm{TO}_{X}$ \\
\hline 637 & 638 & $(\mathrm{LO}+\mathrm{TO})_{\mathrm{L}}$ \\
\hline 666 & 668 & $2 \mathrm{LO}_{X}$ \\
\hline \multirow[t]{2}{*}{685} & 693 & $2 \mathrm{LO}_{\mathrm{L}}$ \\
\hline & 700 & $2 \mathrm{LO}_{\Gamma}$ \\
\hline
\end{tabular}

Raman spectra of this study for natural sphalerite are compared with published data for synthetic sphalerite [63]. Natural sphalerite (this study): sample DT-20.

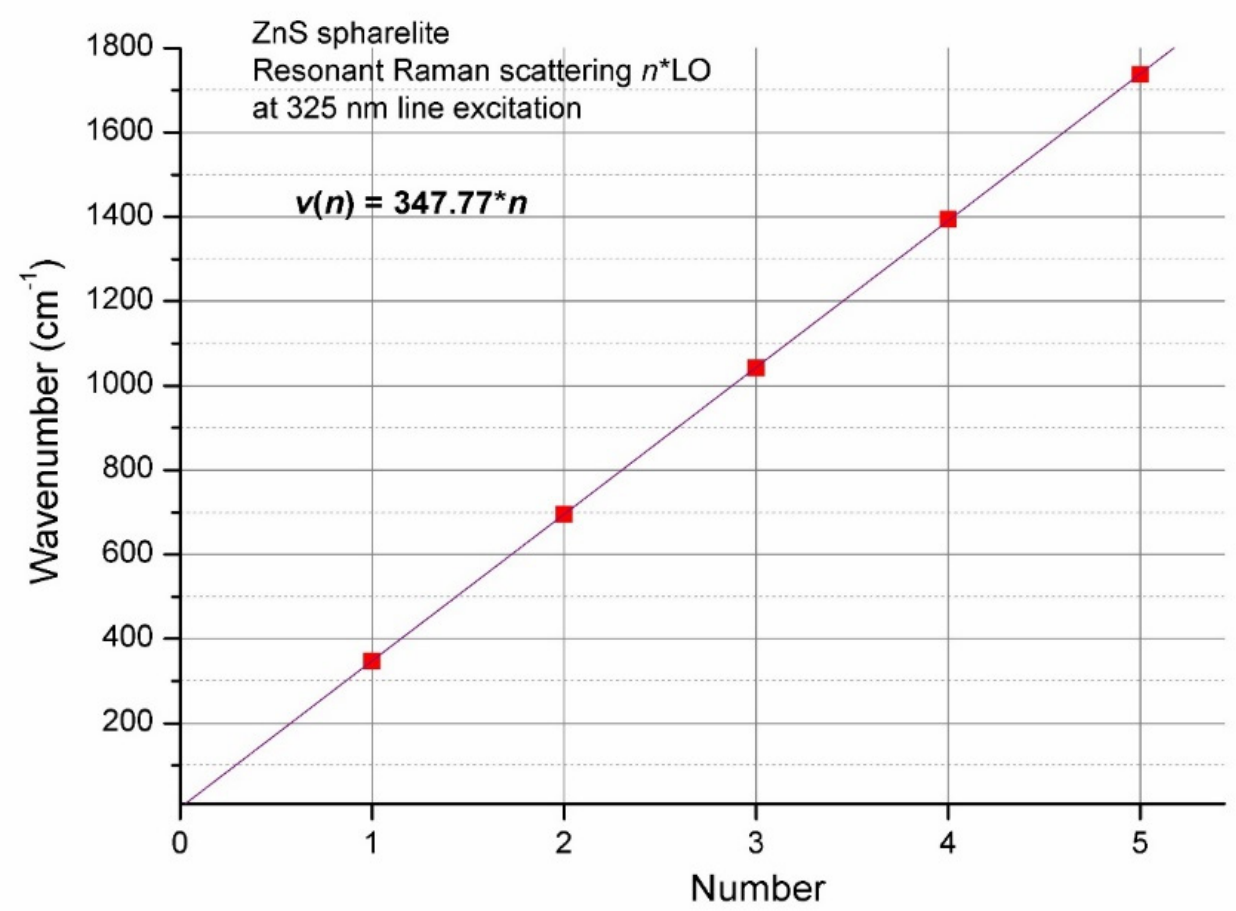

Figure 13. Wavenumber of strong bands $(n \mathrm{LO})$ in resonant Raman spectra of CM sphalerite, at 325-nm excitation, as a function of the number $n$ of repeated basic tone reflections (LO mode $348 \mathrm{~cm}^{-1}$ ). 


\section{Discussion}

5.1. Fractionation of Metals (Fe, $\mathrm{Mn}, \mathrm{Cd}, \mathrm{Hg}, \mathrm{In}, \mathrm{Ga})$ and Chalcogenides (Se, Te, $\mathrm{As}, \mathrm{Sb}$ ) in $\mathrm{ZnS}$ : a Brief Overview

Sphalerite (cubic) and würtzite (hexagonal) ZnS polymorphs have only a minor enthalpy difference (from 2 to $13 \mathrm{~kJ} \mathrm{~mol}^{-1}$ at ambient conditions). This small difference alone cannot account for greater stability of the zinc blende structural type and its wide spread in both natural and synthetic ZnS systems, given that the würtzite type is more stable in the case of CdS [65]. In the latter publication, the stability of the two structure types was explained proceeding from the respective interatomic potentials of $\mathrm{ZnS}$ and $\mathrm{CdS}$ and from their ability to reproduce many properties of sphalerite, würtzite, greenockite, and hawleyite $\left(\mathrm{CdS}_{\mathrm{cub}}\right)$. The same approach can be useful to understand the fractionation of $\mathrm{Zn}, \mathrm{Cd}$, and some other TE in natural sulfidic assemblages containing $\mathrm{ZnS}$ and/or CdS.

The sphalerite structure can accommodate many ions comparable in size with tetrahedrally coordinated $\mathrm{Zn}^{2+}$ and has an affinity to tetrahedral coordination due to $s p 3$ hybrid orbitals $[65,66]$. This hybridization type is typical of $\mathrm{Cd}^{2+}, \mathrm{Hg}^{2+}, \mathrm{Ga}^{3+}, \mathrm{In}^{3+}$, and $\mathrm{Ag}^{+}$, and, possibly, also of $\mathrm{Cu}^{+}$, $\mathrm{Tl}^{3+}, \mathrm{Ge}^{4+}$, and $\mathrm{Sn}^{4+}$. Unlike the isovalent substitutions $\mathrm{Zn}^{2+} \rightarrow \mathrm{Me}^{2+}(\mathrm{Me}=\mathrm{Cd}, \mathrm{Fe}, \mathrm{Mn}, \mathrm{and} \mathrm{Hg})$, other cations like $\mathrm{In}^{3+}, \mathrm{Ga}^{3+}$, and $\mathrm{Ag}^{+}$incorporate into $\mathrm{ZnS}$ through heterovalent schemes. The isovalent substitutions produce diverse multicomponent solid solutions, namely FeS, CdS, HgS, and ZnSe, that have a cubic zinc blende-type structure: rudashevskyite $\left(\mathrm{FeS}_{\mathrm{cub}}\right)$, hawleyite $\left(\mathrm{CdS}_{\mathrm{cub}}\right)$, metacinnabar $\left(\mathrm{HgS}_{\mathrm{cub}}\right)$, and stilleite $\left(\mathrm{ZnSe}_{\mathrm{cub}}\right)$ [1,67-69]. Alabandite $\left(\mathrm{MnS}_{\mathrm{cub}}\right)$ belongs to the galena structural type, and the $(\mathrm{Zn}, \mathrm{Mn}) \mathrm{S}_{\text {cub }}$ solid solutions are more restricted than $(\mathrm{Zn}, \mathrm{Fe}) \mathrm{S}_{\mathrm{cub}}$. The $(\mathrm{Zn}, \mathrm{Fe}, \mathrm{Mn}) \mathrm{S}_{\text {cub }}$ homogeneous solid solutions may contain up to $\sim 4 \mathrm{wt} \%$ (7 mol\%) Mn. Rambergite $\left(\mathrm{MnS}_{\text {hex }}\right)$ has the würtzite-type structure, and $(\mathrm{Zn}, \mathrm{Mn}) \mathrm{S}$ crystals adopt this structure type or contain separate domains of sphalerite and würtzite, where the share of $\mathrm{MnS}$ endmember in $(\mathrm{Zn}, \mathrm{Mn}) \mathrm{S}$ solid solution can reach $50 \mathrm{~mol} \%$ [70]. Therefore, natural sphalerites commonly contain a few $\mathrm{wt} \%$ of Fe or Mn, and smaller amounts of $\mathrm{Cd}, \mathrm{Hg}$, and/or Se. The three latter elements, being common impurities, rarely reach high contents in natural sphalerites. Such 'anomalous' sphalerites are restricted to particular environments. Chemically pure ZnS sphalerite or würtzite are of scarce natural occurrence.

Iron and manganese can easily incorporate into the sphalerite structure at higher temperatures [61,71]. They reach the highest contents in meteorite sphalerites (25.3-32.1 wt\% Fe or 42-50 mol\% FeS) [72] and in ultra-high-temperature contact spurrite-mervinite marbles (19.0-20.7 wt\% Fe; 4.0-5.9 wt\% Mn; $\mathrm{Zn}_{0.55-0.57} \mathrm{Fe}_{0.32-0.35} \mathrm{Mn}_{0.07-0.10} \mathrm{~S}$ ) [73]. The incorporation of large amounts of $\mathrm{Mn}$ stabilizes the würtzite structure [71], and würtzite from the contact marbles contains similar concentrations of Fe and Mn: 12.0 to $19.1 \mathrm{wt} \%$ and 16.8 to $18.3 \mathrm{wt} \%$, respectively $\left(\mathrm{Zn}_{0.39-0.46} \mathrm{Fe}_{0.19-0.31} \mathrm{Mn}_{0.28-0.30} \mathrm{~S}\right)$ [73].

The contents of $\mathrm{Cd}, \mathrm{Hg}$, and Se in sphalerite are very high only in the geochemical environments where these elements and their ratios $(\mathrm{Cd} / \mathrm{Zn}, \mathrm{Hg} / \mathrm{Zn}$, or Se/S) are high as well. This is the case of active fumaroles at Kudriavy volcano (Iturup Island, Russia) with unique In, Cd, Zn, Cu, Mo, $\mathrm{Re}$, and Bi sulfide mineralogy [74,75] and two types of ZnS-CdS solid solutions: $(\mathrm{Zn}, \mathrm{Cd}, \mathrm{Fe}) \mathrm{S}_{\text {cub }}$ containing up to $8 \mathrm{~mol} \% \mathrm{CdS}$ and $(\mathrm{Zn}, \mathrm{Cd}, \mathrm{Fe}) \mathrm{S}_{\text {hex }}-(\mathrm{Cd}, \mathrm{ZnFe}) \mathrm{S}_{\text {cub }}$ with $\sim 17-100 \mathrm{~mol} \% \mathrm{CdS}$. Although hawleyite, a cubic CdS, is stable above $525^{\circ} \mathrm{C}$ [76], the contents of $\mathrm{Cd}$ in $\mathrm{ZnS}_{\text {cub }}$ become much lower at higher temperatures (Figure 14) [74,77]. Nevertheless, restricted solid solutions of $\left(\mathrm{Zn}_{1-\mathrm{x}} \mathrm{Cd}_{\mathrm{x}}\right) \mathrm{S}_{\mathrm{cub}}$ can be synthesized by chemical transport reactions in the temperature range from 700 to $1100{ }^{\circ} \mathrm{C}$ [71]. In natural environments, at higher temperatures, cadmium in Cd-rich growth media becomes ever more prone to form its own phases rather than being a member of solid solutions with Zn counterparts, which is true for both sulfide and oxide compounds $[19,65]$.

Mercury is often stored in sphalerite from complex sulfosalt-HgS deposits, up to the composition $\left(\mathrm{Zn}_{0.752} \mathrm{Hg}_{0.248}\right) \mathrm{S}$ [78], e.g., $\mathrm{Hg}$ content in sphalerite from Eskay Crek, Canada, reaches $16.4 \mathrm{wt} \%$ [79]). Some Hg-rich sphalerites also contain up to $7.96 \mathrm{wt} \% \mathrm{Cd}$ [78].

Selenium reaches thousands of ppm in sphalerite from black shale deposits [80] and $0.12 \mathrm{wt} \% \mathrm{Se}$ was reported from sphalerite coexisting with other Se-bearing sulfides [81]. Note in this respect that 
Se in the Muwaqqar Fm. 'oil shales' resides mostly in authigenic pyrite (6535 ppm Se) rather than in authigenic ZnS (282 ppm) (Table 3).

Sphalerites with high indium contents are very rare but are known from high-temperature fumarolic crusts in Kudriavy volcano and from the Toyoha Cu-Zn-In epithermal deposit (Japan), where In reaches $4.75 \mathrm{wt} \%$ and $5.9 \mathrm{wt} \%$, respectively $[1,74,75]$. Both sphalerite and würtzite from Kudriavy volcano underwent heterovalent substitutions $2 \mathrm{Me}^{2+} \rightarrow \mathrm{Cu}^{+}+\mathrm{In}^{3+}(\mathrm{Me}=\mathrm{Zn}, \mathrm{Cd}, \mathrm{Fe})$. The zinc blende structure has a higher storage capacity than würtzite relative to In and $\mathrm{Cu}$ [74]: up to $2.9 \mathrm{wt} \%$ $\mathrm{Cu}$ and $4.75 \mathrm{wt} \% \mathrm{In}$ in sphalerite and $1.3 \mathrm{wt} \% \mathrm{Cu}$ and $2.8 \mathrm{wt} \% \mathrm{In}$ in würtzite, which corresponds to the $\mathrm{Cu} / \mathrm{In}$ molar ratio of about 1 . According to the phase diagram of the $(\mathrm{ZnS})_{3 \mathrm{x}}-\left(\operatorname{In}_{2} \mathrm{~S}_{3}\right)_{1-\mathrm{x}}$ system, the zinc blende-type structure can accommodate up to $10 \%$ of $\operatorname{In}_{2} \mathrm{~S}_{3}$ [71].

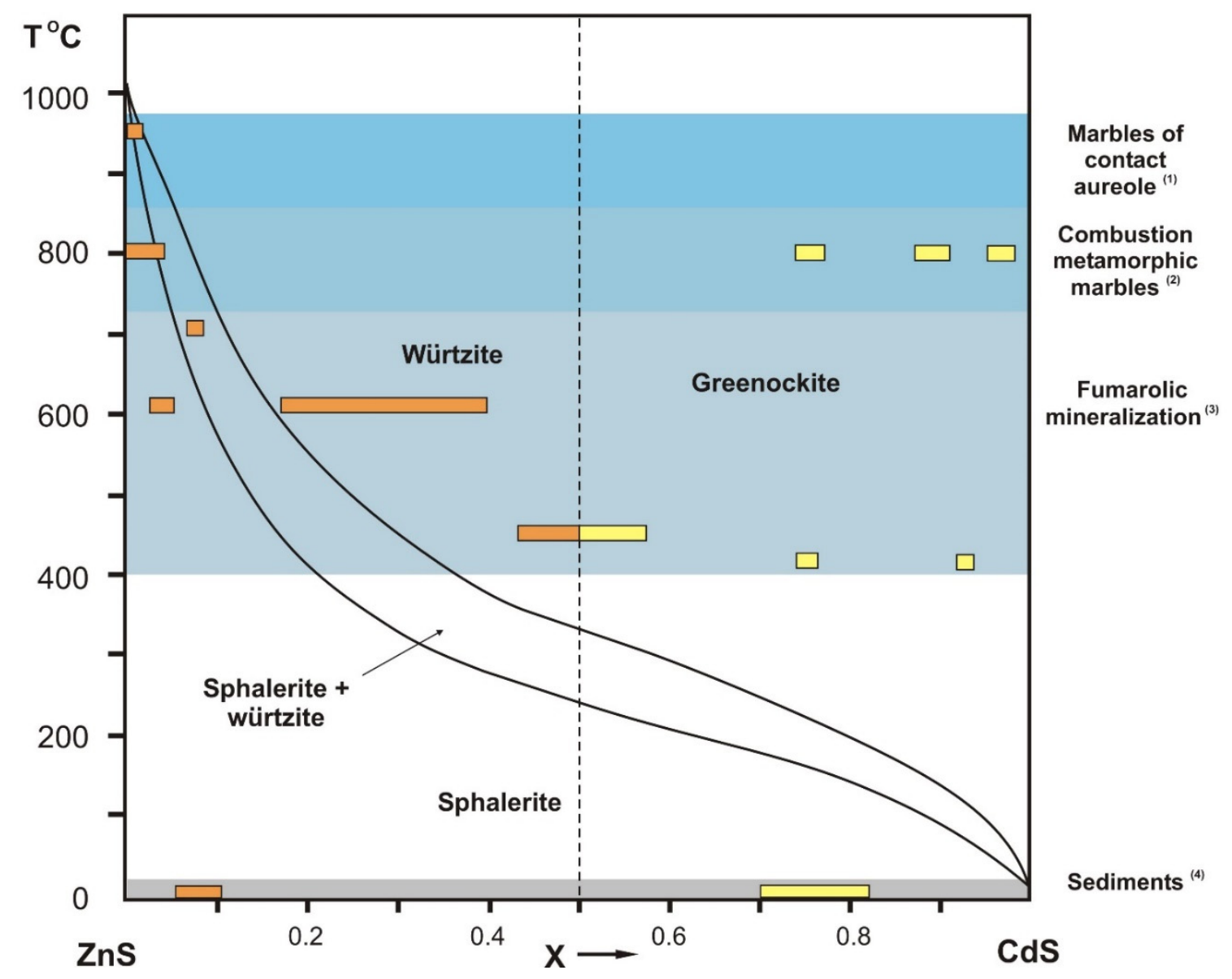

Figure 14. Phase diagram of the system $\mathrm{ZnS}-\mathrm{CdS} ; \mathrm{x}$ - the molar fraction of $\mathrm{Cd}$ in $\mathrm{Zn}_{1-\mathrm{x}} \mathrm{Cd}_{\mathrm{x}} \mathrm{S}$ solid solution. Phase boundaries after [77]. Orange (sphalerite and würtzite) and yellow (greenockite) colors show the composition ranges of sulfides from different rock types: (1) spurrite marbles from Kochumdek contact aureole (Podkamennaya Tunguska Basin, East Siberia), after [73]; (2) CM marbles from Tulul al Hammam area (Daba-Siwaqa complex, Central Jordan), this study; (3) fumarolic mineralization of Kudriavy volcano (Iturup Island, Kuriles), after [74]; (4) immature organic-rich siliceous chalks ('oil shales') of the Muwaqqar Chalk Marl Formation, after [14] and this work.

The tetrahedral coordination of $\mathrm{Ga}^{3+}$ in chalcogenide compounds, with sp3 hybrid orbitals [66], favors the incorporation of $\mathrm{Ga}$ into sphalerite at high temperatures. The amounts of Ga reach $25 \mathrm{~mol} \%$ and $16.3 \mathrm{~mol} \%$ at 900 and $800{ }^{\circ} \mathrm{C}$, respectively, in the Zn-Ga-S system and $27.4 \mathrm{~mol} \%$ Ga at $800{ }^{\circ} \mathrm{C}$ in the $\mathrm{Zn}-\mathrm{Fe}-\mathrm{Ga}-\mathrm{S}$ system [82]. At higher contents of $\mathrm{Ga}$, the $\mathrm{ZnS}-\mathrm{Ga}_{2} \mathrm{~S}_{3}$ system develops triple compounds like $\mathrm{ZnGa}_{2} \mathrm{~S}_{4}, 2 \mathrm{ZnS} \cdot 3 \mathrm{Ga}_{2} \mathrm{~S}_{3}$, or $\mathrm{ZnS} \cdot 3 \mathrm{Ga}_{2} \mathrm{~S}_{3}$ [71]. In spite of the possibility for Ga to incorporate into the zinc blende lattice, natural Ga-rich sphalerites are yet unknown, likely, because $\mathrm{Ga}$ is a trace element 
which rarely exceeds upper crust contents in natural ore-forming systems. The highest Ga contents in sphalerite (up to $366 \mathrm{ppm}$ ) were reported from epithermal deposits in SE Europe and Japan [1].

Although sphalerite is sometimes considered as a carrier of silver, in practice higher Ag concentrations are almost always related to microscopic or submicroscopic inclusions of discrete $\mathrm{Ag}$ minerals [1], which is supported by the eutectic-type phase diagram of the $\mathrm{ZnS}-\mathrm{Ag}_{2} \mathrm{~S}$ system. The ultimate solubility of $\mathrm{Ag}_{2} \mathrm{~S}$ in $\mathrm{ZnS}$ reaches $0.011 \%, 0.022 \%$, and $0.052 \%$ at 600,700 , and $750^{\circ} \mathrm{C}$, respectively, as it was estimated by saturation of solid $\mathrm{ZnS}$ with silver [71].

The substitution of $\mathrm{Zn}^{2+}$ by ions with other hybridization types, such as $\mathrm{Co}^{2+}, \mathrm{Ni}^{2+}, \mathrm{Sb}^{3+}, \mathrm{Mo}^{4+}$, $\mathrm{Pb}^{2+}$, or $\mathrm{Bi}^{3+}$, is strongly limited. Namely, $\mathrm{Ni}$ incorporated into $\mathrm{ZnS}$ is at most $1 \% \mathrm{NiS}$ in hydrothermally synthesized samples [71]. Incorporation of monovalent $\left(\mathrm{Cu}^{+}, \mathrm{Ag}^{+}\right)$, trivalent $\left(\mathrm{In}^{3+}, \mathrm{Ga}^{3+}, \mathrm{Fe}^{3+}, \mathrm{Tl}^{3+}\right)$, and tetravalent $\left(\mathrm{Ge}^{4+}, \mathrm{Sn}^{4+}, \mathrm{Mo}^{4+}\right)$ elements may be evidence of coupled isomorphic substitutions, such as $2 \mathrm{M}^{+}+\mathrm{M}^{2+}+\mathrm{M}^{4+} \rightarrow 4 \mathrm{Zn}^{2+}$ or even more complex schemes [1]. $\mathrm{M}^{3+}$ and $\mathrm{M}^{4+}$ can enter the sphalerite structure due to the formation of donor-acceptor pairs with monovalent ions [83].

As for anionic species, only Se reaching several $w t \%$ has been revealed so far in sphalerite. Confirmations of arsenic-bearing sphalerites in the electron microprobe era have been few [1]. The As concentrations about hundreds of ppm reported for black shale-hosted sphalerite [80] appear to be the highest among natural occurrences known before. According to our data (Table 3), authigenic ZnS modifications from the Muwaqqar Fm. 'oil shales' contain 2255 ppm As. Antimony is not a common impurity in sphalerite, with its highest reliable values most likely ranging within $0.24-0.55 \mathrm{wt} \%$ $\mathrm{Sb}$, except for sphalerite from the epithermal Au-Te Săcărimb deposit (Romania) where it reaches 500-1000 ppm. The contents of Bi in natural sphalerite are moderate, and tellurium is $\leq 0.05 \mathrm{ppm}[1,84]$. We know quantitative estimates only for Te solubility in $\mathrm{ZnS}$ indicating that incorporation of $5 \cdot 10^{-4} \mathrm{wt} \%$ Te into the ZnS system produces inclusions of discrete phase in single crystals of ZnS [71].

Compared to sphalerite from main economic deposits, authigenic ZnS modifications from the Muwaqqar Fm. 'oil shales' show particular combinations of TE signatures (Table 3; Figure 15). In addition to anomalously high $\mathrm{Cd}$ enrichment ( $\sim 6.6$ to $8.1 \mathrm{wt} \%$ on average), they contain up to 7760 ppm Cu, 2145 ppm Sb, 645 ppm Ag, 600 ppm Mo and Pb, and 200 to 345 ppm Ge, Ga, and Hg. At the same time, they are depleted in Se ( 280 ppm) and bear only trace amounts of $\mathrm{Mn}, \mathrm{Tl}$, In, and Bi, while the contents of $\mathrm{Co}, \mathrm{Te}$, and $\mathrm{Au}$ are below detection limits.

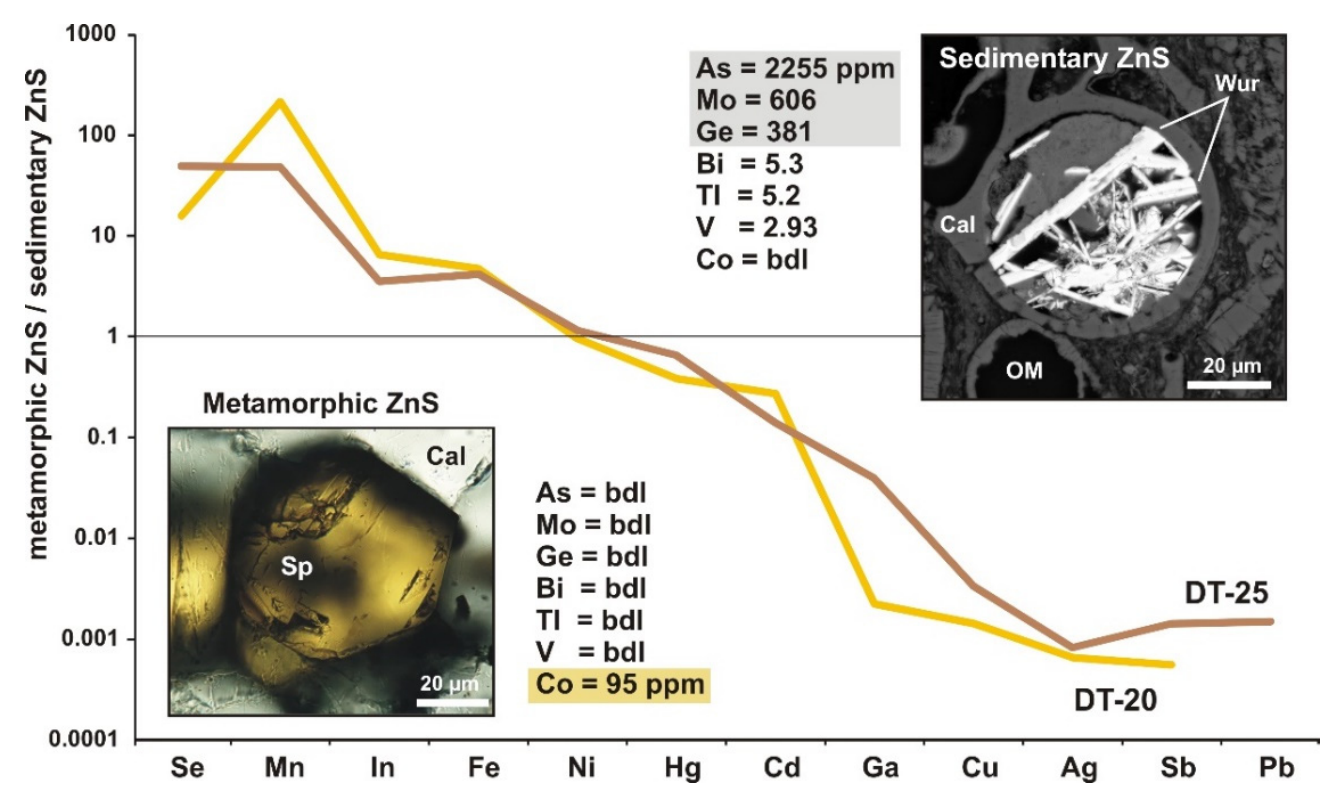

Figure 15. Trace element patterns of combustion metamorphic ZnS (sphalerite), normalized to its authigenic sedimentary precursor ZnS (würtzite and sphalerite); based on LA-ICPMS (Table 3), SEM-EDS and EPMA (Table 2) data. $\mathrm{Cal}=$ calcite, $\mathrm{OM}=$ organic matter, $\mathrm{Sp}=$ sphalerite, $\mathrm{Wur}=$ würtzite. 


\subsection{Marbles: TE Signatures in Sediments and Redox Conditions of Their Annealing}

All Tullul Al Hammam CM marbles share certain geochemical similarity (e.g., high P, Zn, Cd, U; elevated to very high Se, Ni, V, Mo; low Fe, As, Sb; very low Mn, Co, Tl, Hg contents), even though individual samples may be different. The major- and trace-element compositions of the marbles, including high Zn up to $1000-1500$ ppm, with abundant Zn oxide and sulfide mineralization (Table 1), are likewise similar because they formed by high-temperature low-pressure alteration of the same sedimentary protoliths $[14,19,20,22]$. The distribution of redox-sensitive elements (RSEs) in both Muwaqqar Fm. sediments and CM marbles shows high Zn concentrations, while Cd varies from low or moderate to extremely high $[4,14,19,20,51]$. This feature becomes prominent upon comparison of 20-40 g replicas of the same CM marble sample (nugget effect). According to our data, the difference is within 1.04-1.16 times for $\mathrm{Zn}$ and about two or three times for $\mathrm{Cd}$. These patterns, which have been also found common to MVT host rocks, may explain the large range of Cd contents in sphalerite $[1,14,85]$. $\mathrm{Se}, \mathrm{Ni}$, and $\mathrm{Cu}$ in $\mathrm{CM}$ marbles are likewise unevenly distributed (Table 3), and the nugget effect in metamorphic rocks can thus be attributed to an uneven distribution of authigenic pyrite (the primary host of the respective elements) in the precursor sediments.

The formation of oxide- or chalcogenide-dominated mineral assemblages of $\mathrm{Zn}, \mathrm{Cd}, \mathrm{Ni}$, and $\mathrm{Cu}$ during CM events was mainly governed by local redox conditions. The oxygen supply during burning of disseminated OM and annealing of sediments controlled the activity ratio of sulfidic and sulfate sulfur. At any redox regime, CM alteration of 'oil shales' was accompanied by their considerable decarbonation and total dehydration, as well as by partial loss of sulfur and some other highly volatile elements, such as Cd, Hg, and As. Most of the Tulul al Hammam marbles store Zn in oxides [19,20]. The prevalence of oxygenated $\mathrm{Zn}$ compounds is reasonable because combustion metamorphism commonly occurs at an excess of the oxidant (air oxygen) over fuel, with oxygen fugacity $\left(f_{\mathrm{O} 2}\right)$ at or above the magnetite/hematite buffer [86-88].

$\mathrm{CM}$ alteration of the sedimentary rocks rarely occurs in reduced conditions, at an excess of fuel over oxidant. Nevertheless, diverse sulfides $[4,16,19,20]$, such as zoharite $\left((\mathrm{Ba}, \mathrm{K})_{6}(\mathrm{Fe}, \mathrm{Cu}, \mathrm{Ni})_{25} \mathrm{~S}_{27}\right)[12]$, K-Fe-Cu-Ni sulfide [5], ZnS, and Ag-S compounds [19,20]; native elements [6]; and phosphides, including halamishite $\left(\mathrm{Ni}_{5} \mathrm{P}_{4}\right)$, negevite $\left(\mathrm{NiP}_{2}\right)$, transjordanite $\left(\mathrm{Ni}_{2} \mathrm{P}\right)$, and polekhovskyite $\left(\mathrm{MoNiP}_{2}\right)$, have been progressively more often found in CM rocks of the Mottled Zone [6-11].

Widespread apatite-group minerals record the redox conditions during CM alteration of P-rich sediments [89,90]. Zn-Cd oxide mineralization predominates in the marbles with rock-forming fluorapatite-fluorellestadite $\left.\left(\mathrm{Ca}_{5}\left(\mathrm{SiO}_{4}\right)_{1.5}\left(\mathrm{SO}_{4}\right)_{1.5} \mathrm{~F}\right)-\mathrm{Ca}_{5}\left(\mathrm{PO}_{4}\right)_{3} \mathrm{~F}\right)$ solid solutions, with coupled $\left(\mathrm{SiO}_{4}\right)^{4-}{ }_{-}$ and $\left(\mathrm{SO}_{4}\right)^{2-}$ - groups [18-20,91,92]. In sphalerite marbles, rock-forming $\mathrm{Ca}$ phosphate is $\left(\mathrm{SiO}_{4}\right)$-substituted carbonate-fluorapatite poor in sulfate sulfur $\left(0.49-1.85 \mathrm{wt} \% \mathrm{SO}_{3}\right.$ in fluorapatites from DT samples) (Figure 6; Table 4). The pristine and pelletal phosphorites from the Muwaqqar Fm. sediments composed of cryptocrystalline carbonate-fluorapatire (francolite) have a composition of 51.4-52.2 wt\% CaO, 36.0-37.7 wt\% $\mathrm{P}_{2} \mathrm{O}_{5}, 0.5-1.3 w t \% \mathrm{SO}_{3}, 1.6-3.9 w \mathrm{t} \% \mathrm{~F}, 0.2-0.5 \mathrm{wt} \% \mathrm{Cl}$, up to $1.5 \mathrm{wt} \% \mathrm{SrO}$, and $\mathrm{wt}_{\%} 0.3-0.9 \mathrm{Na}_{2} \mathrm{O}[14,30]$, which is consistent with the content of $\mathrm{SO}_{3}$ in $\mathrm{CM}$ carbonate-fluorapatite. Formation of a $\left(\mathrm{SO}_{4}\right)^{2-}$-rich fluorellestadite member obviously requires additional sulfur sources, such as S-rich II-type kerogen and authigenic sulfides $\left(\mathrm{ZnS}\right.$ and $\left.\mathrm{FeS}_{2}\right)$, in the Muwaqqar Fm. sediments [14].

Conversion of sulfide sulfur to sulfate occurred by oxidative combustion of dispersed S-rich kerogen and sulfides. The same process can destroy the primary depots of TE and thus liberate metals that were immobilized in authigenic sulfides $(\mathrm{Zn}, \mathrm{Cd}, \mathrm{Fe}, \mathrm{Ni}, \mathrm{Cu}, \mathrm{Mo}, \mathrm{Ag}$ ) or bound with organic matter $(\mathrm{Ni}, \mathrm{V}, \mathrm{Cu})$. During oxidative annealing of 'oil shales', $\mathrm{Zn}, \mathrm{Cd}, \mathrm{Ni}$, and $\mathrm{Cu}$ are immobilized in simple or double (Ca- or Mg-bearing) oxides, or less often form complex compounds, such as tululite [15,18-20,93]. Mo and V oxidize to their highest oxidation states and form the respective anions. Vanadate is mainly consumed by apatite and nabimusaite supergroup minerals [18-20,94]. Molybdate has been found so far as a single mineral of powellite $\left(\mathrm{CaMoO}_{4}\right)$ in Jordanian CM marbles $[4,93]$. Silver is off this trend and mainly occurs as halides or sporadically as sulfide and native Ag [18-20,93]. 
Iron (mainly $\mathrm{Fe}^{+3}$ ) forms complex ferritic phases with $\mathrm{Ca}, \mathrm{Al}, \mathrm{Ti}, \mathrm{Cr}$, and $\mathrm{Zr}$, but $\mathrm{Fe}^{+2}$ compounds are of secondary importance, excepting relatively widespread $(\mathrm{Mg}, \mathrm{Zn}, \mathrm{Ni}, \mathrm{Cu}, \mathrm{Fe}) \mathrm{O}$ solid solutions with $\mathrm{Zn}>>$ $\mathrm{Ni} \geq \mathrm{Cu}>\mathrm{Fe}^{2+}[5,6,15,19,38,93,95]$.

Partitioning of iron between different minerals of sphalerite-bearing CM marbles is worth special attention because $\mathrm{Fe}$ is a main carrier of sulfidic sulfur under reducing conditions in ordinary metamorphic rocks [3,73]. Rocks formed at temperatures similar to those for the Jordanian CM marbles contain other Fe-bearing sulfides, besides predominant pyrrhotite. This is specifically the case of the Kochumdek contact aureole with abundant pyrrhotite, less-abundant Fe-rich sphalerite and rasvumite, and sporadic Mn- and Fe-rich würtzite [73]. In meteorites, pyrrhotite is likewise a predominant sulfide while sphalerite has the greatest Fe enrichment [72]. Unlike these, FeS or $\mathrm{FeS}_{2}$ have been found in none of the Tullul Al Hammam marble sample. The lack of iron sulfides and low Fe contents in the CM sphalerites may be due to a joint effect of low Fe contents in precursor 'oil shales' and specific redox conditions during their CM alteration. The original depletion in Fe led to a predominance of authigenic $\mathrm{ZnS}$ over $\mathrm{FeS}_{2}$ at prominent excess of sulfide sulfur mainly stored in kerogen [14]. As for the redox regime, both sulfide-bearing and sulfide-free assemblages that form during CM alteration of the Muwaqqar Fm. sediments contain predominant $\mathrm{Fe}^{+3}$ compounds, mainly complex calcium ferrites and aluminate-ferrites.

The temperature and redox conditions of combustion metamorphism are also indicated by complex $\mathrm{Ca}-\mathrm{Fe}-\mathrm{Ni}-\mathrm{Cu}$ oxychalcogenides coexisting with sphalerite. Oxysulfide compounds are extremely rare in nature $[15,16,96]$ but are ordinary intermediates during calcination roasting of Fe-bearing sulfides. Among these, $\mathrm{Ca}-\mathrm{Fe}$ oxysulfides $\mathrm{Ca}_{3} \mathrm{Fe}_{4} \mathrm{~S}_{3} \mathrm{O}_{6}$ and $\mathrm{CaFeSO}$ are the most common and have the largest stability fields. The composition, structure, and stability fields of $\mathrm{Ca}, \mathrm{Fe}^{2+}, \mathrm{Fe}^{3+}, \mathrm{Zn}, \mathrm{Cu}$, and $\mathrm{Ni}$ oxysulfides have been well documented because they are key intermediates in industrial carbothermal metal reduction and/or reduction-calcination reactions in metal-sulfur-calcium-oxygen (M-S-Ca-O) systems. A complete set of phases at the intermediate reduction stage of complex $\mathrm{Zn}, \mathrm{Cu}$, and $\mathrm{Ni}$ sulfide ores includes metals; Fe-Ni alloys; ferrites and aluminate-ferrites of $\mathrm{Ca}, \mathrm{CaO}$, or $\mathrm{CaS}$; as well as $\mathrm{Ca}$ and Fe oxysulfides with different stoichiometry [97-104]. The industrial process is performed under ambient pressure and 900 to $1250{ }^{\circ} \mathrm{C}$, in the presence of carbon $\left(\mathrm{C}^{0}\right), \mathrm{CaO} / \mathrm{CaCO}$, and/or $\mathrm{CaSO}_{4}$. The redox conditions of the process, which is controlled by partial gas pressure of $\mathrm{H}_{2} \mathrm{~S} / \mathrm{SO}_{2}$ and $\mathrm{CO} / \mathrm{CO}_{2}$ (commonly expressed as $\mathrm{SO}_{2}$ or $\mathrm{CO}$ gas potential or fugacity), provides selective oxidation of $\mathrm{Fe}^{+2}$ to $\mathrm{Fe}^{+3}$. Iron interacting with $\mathrm{CaO}$ binds into oxide (mainly Ca ferrites) and oxysulfide phases and thus becomes separated from the reduced forms of $\mathrm{Zn}, \mathrm{Cu}$, and $\mathrm{Ni}$ occurring as sulfide or metal phases [97-102].

In the $\mathrm{Cu}-\mathrm{Fe}-\mathrm{Ca}-\mathrm{S}-\mathrm{O}$ system, no metallic phases have been observed below $850{ }^{\circ} \mathrm{C}$ because both Fe and $\mathrm{Cu}$ form complex oxysulfides. Under higher temperatures $\left(>900{ }^{\circ} \mathrm{C}\right)$ and $f_{\mathrm{SO} 2}$ between $10^{-8}$ and $10^{-4} \mathrm{~atm}, \mathrm{Cu}^{0}$ begins to separate and coexist with the $\mathrm{CaFeSO}$ phase [99]. Nickel needs a higher $f_{\mathrm{SO} 2}\left(10^{-5.5}-10^{-5} \mathrm{~atm}\right.$ at $850{ }^{\circ} \mathrm{C}$ and $10^{-4} \mathrm{~atm}$ at $\left.1000^{\circ} \mathrm{C}\right)$ for reduction and separation from Fe, which occurs as $\mathrm{Fe}^{+2}$ or $\mathrm{Fe}^{+3}$ compounds. The very narrow range of $f_{\mathrm{SO} 2}$ where $\mathrm{Ni}$ can reduce selectively requires fine regulation of $f_{\mathrm{SO} 2}$ and thus impedes $\mathrm{Ni}$ separation from Fe. Therefore, (Ni,Fe) alloys frequently occur instead of metallic Ni. Carbothermal reduction using CaO doping at 950 to $1050{ }^{\circ} \mathrm{C}$ can make $\mathrm{Ni}^{0}$ separation from Fe more efficient, because iron preferentially reacts with lime forming $\mathrm{Ca}_{3} \mathrm{Fe}_{4} \mathrm{~S}_{3} \mathrm{O}_{6}$ and $\mathrm{CaFeSO}$ phases and more complex Ca-Fe-Ni oxysulfides [99,105]. Separation of Ni from Ca-Fe oxysulfides can likewise be achieved by using carbon monoxide as a reducing agent [105].

The discovered assemblages of sphalerite, brownmillerite, Ca ferrites, and mixed oxychalcogenides (with S and Se) of Ca-Fe-Ni-Cu with exsolution textures and micrometer-sized inclusions of $\mathrm{Ni}, \mathrm{Ag}$, and $\mathrm{Zn}$ phases indicate that carbothermal reduction of metals and reduction-calcination reactions in Me-S-Se-Ca-O systems took place in a natural environment. Oldhamite (CaS), Ca ferrites, and Ca-Fe oxysulfides can coexist at $727^{\circ} \mathrm{C}$ in the absence of Fe sulfides, native elements, and magnetite within a very restricted field of the phase diagram of the Ca-Fe-S-O system [97]. It allows one to constrain some physicochemical parameters of the natural process. Theoretically, the FeS-to-CaO ratio of the reacting 
mixture during carbothermal metal reduction should be in the range 0.3-0.5. Since the FeS/CaO ratio in bulk iron-depleted sediments is obviously $<0.3$, oxychalcogenide mineralization in CM rocks is restricted to local spots with the highest contents of S-rich kerogen, Zn, and Fe sulfides in the precursor sediments. Oxygen partial pressure $\left(f_{\mathrm{O} 2}\right)$ can be estimated as $\sim 10^{-20} \mathrm{~atm}$ and the respective $f_{\mathrm{SO} 2}$ as $\sim 10^{-10} \mathrm{~atm}$. No metallic phases have been observed in the Cu-Fe-Ca-S-O system below $850^{\circ} \mathrm{C}$ because both $\mathrm{Fe}$ and $\mathrm{Cu}$ form complex oxysulfide compounds. Under higher temperatures $\left(>900{ }^{\circ} \mathrm{C}\right)$ and lower oxygen fugacity ( $f_{\mathrm{SO} 2}$ between $10^{-8}-10^{-4} \mathrm{~atm}$ ), $\mathrm{Cu}^{0}$ begins to separate, and coexists with the $\mathrm{CaFeSO}$ phase [99]. These estimates are consistent with the absence of native element species in sphalerite-bearing marbles.

\subsection{Trace-Element Patterns of CM Sphalerite}

Sphalerite is an important host mineral for a wide range of trace elements in CM rocks (Tables 3, 9 and 10), but only $\mathrm{Cd}$ and Se reach abnormally high values of $\sim 14 \mathrm{wt} \%$ and $\sim 6.7 \mathrm{wt} \%$, respectively. Both elements are inherited by $\mathrm{CM}$ sphalerite from authigenic sulfide minerals: $\mathrm{Cd}$ from $(\mathrm{Zn}, \mathrm{Cd}) \mathrm{S}$ modifications and Se from pyrite (Tables 2 and 3) [4,14].

Cadmium in the $\mathrm{CM}$ sphalerite that resulted from the clinkering of $\mathrm{Zn}$ - and $\mathrm{Cd}$-enriched calcareous marine sediments is $2.01 \mathrm{wt} \% \mathrm{Cd}$ on average $(\mathrm{N}=200)$ (Tables 3, 9 and 10; Figure 10). This value is much above the common average of $\mathrm{Cd}$ contents in sphalerite from economic deposits $(0.25-1.1 \mathrm{wt} \%$ $\mathrm{Cd}$ [1]) and is commensurate with the highest concentrations in that from MVT ores [106], e.g., $1.4 \mathrm{wt} \%$ $\mathrm{Cd}$ in sphalerite from the Niujiaotang zinc deposit in the Guizhou province of China [85]. Note that the Muwaqqar Fm. marly chalks were considered as immature precursors of MVT deposits [14].

Cadmium contents are lower and the $\mathrm{Zn} / \mathrm{Cd}$ ratios are higher than in the precursor sediments in the greatest number of the CM marble samples we studied. Namely, the average $\mathrm{Zn} / \mathrm{Cd}$ ratio is 16 for the marbles and 11 for the Muwaqqar Fm. marly chalks (Table 1). This difference is due to the high volatility of $\mathrm{Cd}$ and is well known for combustion of fossil fuels and clinkering of carbonate rocks [14,19,107-109]. Although much Cd becomes lost during annealing of sediments, CM marbles often contain large amounts of cadmium exceeding the bulk sphalerite storage capacity and also bear other $\mathrm{Cd}$ accessories, such as $(\mathrm{Ca}, \mathrm{Cd}) \mathrm{O}$, tululite, greenockite, or very rarely cadmoselite.

$\mathrm{CM}$ sphalerites are much more depleted in $\mathrm{Cd}$ than authigenic sphalerite or würtzite, with average Cd contents of about $9 \mathrm{wt} \%\left(\mathrm{Zn}_{0.83-0.88} \mathrm{Cd}_{0.07-0.09} \mathrm{Fe}_{0.01} \mathrm{Cu}_{0.01} \mathrm{~S}\right.$ sphalerite; $\mathrm{Zn}_{0.85-0.88} \mathrm{Cd}_{0.08} \mathrm{Fe}_{0.01} \mathrm{~S}$ würtzite; Tables 2, 3, 9 and 10; Figures 10, 14 and 15). Our collection includes a single marble sample (TH-74) with a $\mathrm{Zn} / \mathrm{Cd}$ ratio as low as 4.4 , which contains sporadic sphalerite with an erratic value of $\mathrm{Cd}$ reaching $\sim 14 \mathrm{wt} \%$, and minor greenockite. This specific case illustrates the general trend that the amount of $\mathrm{Cd}$ incorporated into the $\mathrm{ZnS}_{\text {cub }}$ lattice decreases dramatically as the formation temperature increases, while complete solid solution exists between the $\mathrm{ZnS}$ and CdS hexagonal polymorphs $[25,71]$.

The $(\mathrm{Zn}, \mathrm{Cd}) \mathrm{S}-\mathrm{T}\left({ }^{\circ} \mathrm{C}\right)$ diagram (Figure 14$)$ illustrates a transition from Cd-rich low-temperature authigenic sphalerite to ultrahigh-temperature Cd-depleted sphalerite from the Kochumdek contact marbles $\left(\mathrm{T} \geq 900{ }^{\circ} \mathrm{C}\right)$. The $\mathrm{ZnS}_{\text {cub }}$ compositions with moderate $\mathrm{Cd}$ contents, which represent high-temperature $\left(800-850^{\circ} \mathrm{C}\right)$ sphalerite from the Jordanian $\mathrm{CM}$ marbles and those from volcanic fumaroles in the Kuriles $\left(600-750{ }^{\circ} \mathrm{C}\right)$, fall in the middle of the same trend. As it was found out earlier for a Zn-Cd-S-Se system at 1000 and $800{ }^{\circ} \mathrm{C}$ [25], zinc blende-type structure exists only in Zn-rich compositions of: $\leq 20 \mathrm{~mol} \% \mathrm{CdS}$ on the ZnS-CdS side and $\leq 40 \mathrm{~mol} \%$ CdSe on the ZnSe-CdSe side, whereas most compounds have a würtzite-type structure. The stability field of the $\left(\mathrm{Zn}_{1-\mathrm{x}} \mathrm{Cd}_{\mathrm{x}}\right)\left(\mathrm{S}_{1-\mathrm{x}} \mathrm{Se}_{\mathrm{x}}\right)$ hexagonal phase expands with temperature from $22 \mathrm{~mol} \% \mathrm{CdSe}$ at $600{ }^{\circ} \mathrm{C}$ to $\sim 10 \mathrm{~mol} \% \mathrm{CdS}$ at $900{ }^{\circ} \mathrm{C}$ [71]. Since the CdS-CdSe diagram is of type I Roozeboom with unlimited miscibility among the components [71], the $(\mathrm{Cd}, \mathrm{Zn})(\mathrm{S}, \mathrm{Se})$ solid solutions found in the Jordanian CM marbles can be considered as regular ones between $\mathrm{CdS}_{\text {hex }}$ and $\mathrm{CdSe}_{\text {hex }}$ (Table 11). Unfortunately, the sizes of greenockite and cadmoselite crystals are too small to characterize their structure directly. 
Iron is almost always present in natural sphalerite, varying from trace amounts to $50 \mathrm{~mol} \%$ FeS (in meteorites only [72]). Commonly, Fe contents in sphalerite increase progressively with the crystallization temperature. High Fe and Mn contents reaching $\sim 19 \mathrm{wt} \%$ and $\sim 6.5 \mathrm{wt} \%$, respectively, were observed in sphalerite coexisting with alabandite and pyrrhotite from ultra-high-temperature contact metamorphic spurrite-merwinite marbles, whereas würtzite in these marbles contains more manganese and less iron ( $17 \mathrm{wt} \%$ against $\sim 8.5 \mathrm{wt} \%$, respectively) [73]. Quite high Fe contents were also reported for sphalerite from Fe-rich host rocks, namely hedenbergite skarn [1]. Low iron contents in Jordanian CM sphalerite, $1.44 \mathrm{wt} \%$ Fe on average $(\mathrm{N}=200)$ (Figure 10), result mainly from low $\mathrm{Fe}_{\text {total }}$ in the marbles and its preferable fractionation as $\mathrm{Fe}^{3+}$ in complex Ca-rich oxides (brownmillerite, srebrodolskite, and other Ca ferrites).

Manganese concentrations in the CM sphalerite are from a few hundred to $1700 \mathrm{ppm}$, while Co contents are an order of magnitude lower (Table 3). The Mottled Zone CM assemblages lack Mn- and Co-bearing minerals because both elements had very low contents in the precursor sediments. Manganese contents of around $3 \mathrm{wt} \% \mathrm{MnO}$ were measured only in tululite, a complex Ca-Al-Zn-Cd-Fe-Mn oxide, a single relatively abundant high-temperature $\mathrm{Mn}$ mineral found so far in the Mottled Zone complexes [20], besides accessory Mn-enriched spinel of the $\mathrm{MgFe}_{2} \mathrm{O}_{4}-\mathrm{FeMn}_{2} \mathrm{O}_{4}$ series reported from CM hornfels in the Hatrurim Basin [93,94,110].

Cobalt contents in the CM sphalerite are quite low ( $X_{a v}=124$ ppm for DT-20 and 66 ppm for DT-25). It is tempting to attribute this depletion to the respective depletion of the protoliths, but the same Co contents are common to other natural sphalerites as well. The Co range in natural sphalerites is commonly from 1 to 840 ppm, except for a few skarn deposits containing up to $\approx 1500-2300$ ppm Co [1], though $\mathrm{Co}^{2+}$ and $\mathrm{Fe}^{2+}$ ions are of similar sizes and intermediate compositions with up to $40 \mathrm{~mol} \% \mathrm{CoS}$ exist among CoS-ZnS solid solutions of zinc blende-type structures [1,111]. Taking into account very low Co contents (commonly $<2$ ppm) in the bulk CM marble samples, sphalerite can be expected to be a Co carrier in these rocks.

The contents of nickel (265 ppm) determined by LA-ICPMS in a few quite large homogeneous sphalerite grains from Ni-rich sample DT-25 are within the same range 176-296 ppm as in sample DT-20, which is more depleted in Ni and lacks Ni minerals. The Ni enrichment of the Mottled Zone CM sphalerites is far higher than the $<0.1 \mathrm{ppm}$ to $11.4 \mathrm{ppm}$ values measured by LA-ICPMS in other natural sphalerites [1]. Few experimental data indicate the restricted ability of Ni to incorporate into sphalerite even at $\sim 800-850{ }^{\circ} \mathrm{C}$. Wu et al. (1989) [112] revealed restricted NiS-ZnS solid solutions (up to 3 at. $\% \mathrm{Ni}$ ) and confirmed the sphalerite structure of the $\mathrm{Zn}_{48} \mathrm{Ni}_{2} \mathrm{~S}_{50}$ phase. The presence of exsolution textures provides reliable evidence that the range of NiS-ZnS solid solutions becomes narrower at lower temperatures. Ni shows a similar behavior upon the cooling of complex oxychalcogenides and forms an exsolution network of an Ni-S-Se phase in the matrix of Ca-Fe-Cu oxysulfide (Figure 8). The contents of $\mathrm{Ni}$ in the CM sphalerite are 20 to 30 times as high as those determined by LA-ICPMS in sphalerite from the main economic deposits [1]. Therefore, they appear to be at the highest limit for natural sphalerites.

As it was found out previously [14], type-II kerogen of precursor sediments is the main carrier of $\mathrm{V}$ and $\mathrm{Ni}$ (up to $50 \%$ of bulk Ni content in the rock) along with authigenic pyrite (up to $1.6 \mathrm{wt} \% \mathrm{Ni}$ ). Both sedimentary Ni carriers were involved in the formation of sulfide mineralization during reducing annealing. Note in this respect that trevorite $\left(\mathrm{NiFe}_{2} \mathrm{O}_{4}\right)$ and bunsenite $(\mathrm{NiO})$ have been the only Ni-dominant oxides identified so far in the Mottled Zone CM rocks [7,93,113]. Other oxide accessories with essential Ni contents are spinel-group minerals, periclase, Ca ferrites, and silicoferrites [15,93,114]. On the other hand, more than 10 oxygen-free Ni minerals were found in reducing assemblages from the $\mathrm{MZ}$ complexes: kamacite (Fe,Ni) [6]; numerous Ni- and Fe-Ni-phosphides (schreibersite $\left((\mathrm{Fe}, \mathrm{Ni})_{3} \mathrm{P}\right)$, baringerite $\left((\mathrm{Fe}, \mathrm{Ni})_{2} \mathrm{P}_{\text {hex }}\right)$, murashkoite $((\mathrm{Fe}, \mathrm{Ni}) \mathrm{P})$, allabogdanite $\left((\mathrm{Fe}, \mathrm{Ni})_{2} \mathrm{P}_{\text {rhomb }}\right)$, zuktamrurite $\left((\mathrm{Fe}, \mathrm{Ni}) \mathrm{P}_{2}\right)$, halamishite $\left(\mathrm{Ni}_{5} \mathrm{P}_{4}\right)$, negevite $\left(\mathrm{NiP}_{2}\right)$, transjordanite $\left(\mathrm{Ni}_{2} \mathrm{P}\right)$, polekhovskyite $\left(\mathrm{MoNiP}_{2}\right)$, nazarovite $\mathrm{Ni}_{12} \mathrm{P}_{5}$ [6,7,9-11,115-118], millerite and/or mäkinenite ( $\mathrm{Ni}(\mathrm{S}, \mathrm{Se})$ ) [4,16], zoharite [12], and pentlandite; as well as a number of unidentified $\mathrm{Ni}$ phases, including $\mathrm{Ni}_{2} \mathrm{As}$ and $\mathrm{K}-\mathrm{Fe}-\mathrm{Cu}-\mathrm{Ni}$ 
sulfide $[5,6,93]$. This long list has been extended with $\mathrm{Ni}$ oxychalcogenides revealed in the Jordanian $\mathrm{CM}$ marbles. Thus, Ni manifests high mineral productivity in CM processes under reducing conditions, but it becomes mainly dispersed as impurity in complex accessory oxides upon oxidative combustion.

Silver shows highly uneven distribution in CM sphalerite and has been found by SEM-EDS (within $1-5 \mathrm{wt} \%$ ) only in grains with microscopic inclusions of Ag minerals (Tables 6 and 7). The LA-ICPMS estimate of $0.42-0.53 \mathrm{ppm} \mathrm{Ag}$ for large homogeneous grains (Table 3) appears to be more reasonable for lattice-bound $\mathrm{Ag}$ and rules out the role of $\mathrm{CM}$ sphalerite as a silver carrier. Meanwhile, the sphalerite structure acquires higher storage capacity with respect to these impurities under peak metamorphic conditions judging by complex exsolution textures with $\mathrm{Cu}, \mathrm{Ni}$, and $\mathrm{Ag}$ discrete minerals.

Unexpectedly, we discovered relatively high mercury contents of 132 to $226 \mathrm{ppm}$ in CM sphalerite. The precursor sediments are extremely poor in $\mathrm{Hg}$, while most mercury becomes lost during annealing, and its bulk content in marbles is orders of magnitude lower $(<0.002$ to $0.025 \mathrm{ppm})$ (Table 1$)$. This discovery has confirmed the inference of Cook et al. (2009) [1] that sphalerite frequently acts as a dominant $\mathrm{Hg}$ carrier (Figure 15).

Average contents of indium in CM sphalerite are within a few ppm but are 10 times higher than in authigenic ZnS (Figure 15). No coupling with $\mathrm{Cu}$ contents appears, possibly, because the concentrations are too low.

Like previous workers [1], we failed to discover an incorporation of $\mathrm{Cu}, \mathrm{Ga}$, and $\mathrm{Pb}$ in $\mathrm{CM}$ sphalerite and to find even trace amounts of $\mathrm{Ge}, \mathrm{Sn}, \mathrm{Mo}, \mathrm{Tl}$, and $\mathrm{Au}$. Judging by the contents of $\mathrm{Ga}$, $\mathrm{Ge}, \mathrm{Mo}, \mathrm{Ag}$, and $\mathrm{Pb}$ (hundreds of ppm) and about $7800 \mathrm{ppm} \mathrm{Cu}$ in the sedimentary $\mathrm{ZnS}$ precursors (Table 3; Figure 15), the TE composition of the high-temperature $\mathrm{ZnS}$ phase became much simpler during annealing. In most of the analyzed samples, sphalerite compositions are uniform both within (no zoning) and between grains, with minor standard deviations in element concentrations (Table 10). The only exception is in the mineral diversity of sample DT-25, which, to a high probability, mirrors the primary heterogeneous distribution of TE hosts in the parent sediment.

Unlike authigenic ZnS, which contain about 2000 ppm As and Sb, CM sphalerites are depleted in both elements (Figure 15). Trace amounts of Bi were detected in authigenic ZnS, but its content is below the detection limit in CM sphalerite. Tellurium is also below the detection limit in both authigenic and metamorphic $\mathrm{ZnS}$. Thus, Se is the only anionic element occurring in a significant concentration in the CM sphalerite (Tables 3, 9 and 10; Figures 10 and 15).

The sphalerites from the Jordanian CM marbles contain at least $0.29 \mathrm{wt} \%$ Se (Table 10; Figure 10), and the Se contents in most of the sphalerite grains we analyzed $(\sim 80 \%)$ are from 0.3 to $1.9 \mathrm{wt} \% \mathrm{Se}$. The concentrations exceeding $2 \mathrm{wt} \%$ Se (reaching $6.68 \mathrm{wt} \%$ ) are restricted to sphalerite associated with $\mathrm{Ni}$ and Ag selenides, as well as with Se-rich complex oxychalcogenides (sample DT-25). SEM-EDS and elemental mapping revealed a homogeneous distribution of Se within individual CM sphalerite grains at notable variations among grains in thin sections.

Although complete solid solutions exist between cubic modifications of $\mathrm{ZnS}$ and $\mathrm{ZnSe}$ (stilleite) under the conditions of high-temperature dry synthesis (800 and $1000{ }^{\circ} \mathrm{C}$ ) [25], the Se concentration in natural sphalerites rarely reaches thousands ppm. They are namely sphalerites from black shales and some skarn or epithermal deposits, which have smooth profiles indicating lattice-bound Se [1]. As in our case, abnormal Se concentrations were reported for sphalerites that were associated with selenides and contained inclusions of Se-bearing minerals revealed by Se spikes in LA-ICPMS profiles [1].

The comparison of TE compositions of authigenic and $\mathrm{CM}$ sulfides reveals moderate Se fractionation in the sphalerite structure under high-temperature $\left(800-850^{\circ} \mathrm{C}\right)$ dry reducing conditions. The assemblages in sample DT-25 demonstrates that Se preferably fractionates into Ni and Ag selenides and complex oxychalcogenides but remains only an impurity in the coexisting sphalerites. Furthermore, the incorporation of large Se amounts ( $>2 \mathrm{wt} \%)$ in sphalerite is rigorously controlled by extremely high bulk Se contents within local rock volumes. However, the content of Se in CM sphalerite becomes $15-50$ orders of magnitude higher than in the Se-poor sedimentary ZnS precursors 
(Figure 15). In the marbles, clusters of selenides; Se-bearing $\mathrm{Zn}, \mathrm{Ni}$, and $\mathrm{Ag}$ sulfides; and $\mathrm{Fe}, \mathrm{Ni}$, and $\mathrm{Cu}$ oxychalcogenides formed at the expense of Se-bearing pyrite and S-rich kerogen clots.

In general, the composition of the Jordanian $\mathrm{CM}$ sphalerite with comparable average concentrations of $\mathrm{Cd}$, Se, and $\mathrm{Fe}$ at $>1 \mathrm{wt} \%$ may be characterized as 'extreme' according to [1]. In the previous sections of this discussion, we considered the structural and chemical reasons for substitutions, ranges of solid solutions, and main trends of TE fractionation in high-temperature dry moderately reducing $\mathrm{CM}$ environments. However, discrimination between lattice-hosted elements and those in nanoand micro-scale inclusions is not always possible, even by SEM, EPMA, and LA-ICPMS techniques [1]. Below, we discuss the distribution patterns of elements in the structure of real sphalerite crystals using the data of single-crystal XRD and Raman spectroscopy.

\subsection{Crystal Structure and Distribution of $C d, F e$, Se, and $O$ in $C M$ Sphalerite}

The lattice parameters of binary mixed compounds often conform to simple regularity and depend

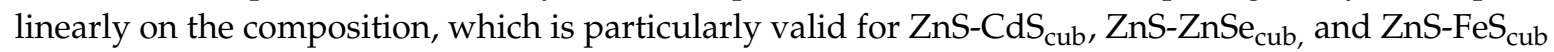
solid solutions $[25,61,66,71]$. However, the miscibility of several substances (multinary mixed phases) on the scale of more than a few per cent is not very frequent, apart from intermetallic systems, and each specific case requires proofs [25].

Single-crystal XRD has provided direct evidence for the distribution of $\mathrm{Cd}$, Fe, and Se in CM sphalerite. The analyzed crystal did not show any additional reflections that would represent inclusions or domains of extraneous phases, whether $\mathrm{Cd}, \mathrm{Fe}$, and Se or O-bearing compounds, such as $\mathrm{ZnO}$ or $\mathrm{Zn}$ sulfates. Thus, the sphalerite is single crystal, and the compound is a multinary mixed phase, with the main impurities randomly distributed in the structure. Following the common practice $[26,119,120]$, we applied the single-crystal XRD method to estimate the amount of oxygen in sphalerite.

The analyzed sphalerite of the complex composition $\mathrm{Zn}_{0.96} \mathrm{Cd}_{0.02} \mathrm{Fe}_{0.02}\left(\mathrm{~S}_{0.946(8)} \mathrm{O}_{0.046(8)} \mathrm{Se}_{0.0074}\right)_{\Sigma 1.00}$ has a smaller unit cell than pure ZnS (5.40852(12) $\AA$ against 5.4094 $\AA$ [56]) (Table 12), explainable only by the substitution of $\mathrm{O}^{2-}(1.40 \AA)$ for $\mathrm{S}^{2-}(1.73 \AA)$. Each revealed substitution is individual, because otherwise they jointly would increase the $a$-parameter of the sphalerite (e.g., $5.411 \AA$ at a mole fraction of 0.02 for $\mathrm{FeS}[60,61,121])$, given that both $\mathrm{Fe}^{2+}$ and $\mathrm{Cd}^{2+}$ are larger than $\mathrm{Zn}^{2+}(0.64,0.78$, and $0.60 \AA$, respectively), while $\mathrm{Se}^{2-}$ likewise has a larger radius $(1.84 \AA)$ than $\mathrm{S}^{2-}(1.73 \AA$ ).

The sphalerite contains around $0.5 \mathrm{~mol} \% \mathrm{ZnO}$, and its $a$-parameter, in the absence of other isomorphic substitutions, should be $5.4077 \AA$ assuming the structure reduction from $5.4081 \AA$ at $0.4 \mathrm{~mol} \% \mathrm{ZnO}$ to $5.4065-5.4070 \AA$ at $0.8 \mathrm{~mol} \% \mathrm{ZnO}$ upon the oxygen-for-sulfur substitution reported by [58]. Its difference from the unit cell of pure $\mathrm{ZnS}$ is slightly compensated by the presence of large $\mathrm{Cd}, \mathrm{Fe}$, and Se ions.

Natural sphalerite of the complex composition $(\mathrm{Zn}, \mathrm{Cd}, \mathrm{Fe})(\mathrm{S}, \mathrm{O}, \mathrm{Se})$ can be expected to crystallize in the zinc blende structure $F \overline{4} 3 m\left(\mathrm{~T}_{\mathrm{d}}{ }^{2}\right)$ (Table 12) by analogy to what was inferred for $\mathrm{Zn}$-dominated $\left(\mathrm{Zn}_{1-\mathrm{x}} \mathrm{Fe}_{\mathrm{x}}\right) \mathrm{S}$ solid solution [61]. However, the Raman spectra of our sample are more complex and contain strong bands at 299, 309, and $331 \mathrm{~cm}^{-1}$ in addition to the predicted bands common to $\mathrm{ZnS}_{\text {cub }}$ (Table 13; Figures 11 and 12).

Theoretical analysis of the solid solution suggests either one- or two-mode transformations between phases. The mineral we studied has an intermediate composition. In the case of one-mode transformation, the composition change leads to respective changes in the frequency of selected bands. This is, for instance, one-mode behavior of olivine $\left(\mathrm{Mg}_{1-\mathrm{x}} \mathrm{Fe}_{\mathrm{x}}\right)_{2}\left[\mathrm{SiO}_{4}\right]$ in an $\mathrm{Fe}-\mathrm{Mg}$ cationic solid solution, where $\mathrm{x}$ is the mole fraction of Fe. At a two-mode phase transition, the frequency of the bands changes continuously or sometimes stepwise. In the latter case, one or more new vibration bands appear at some critical content $x$, usually in the same spectral range, whereby the mode intensity pumps from the initial band to the new band(s) as $x$ changes. The behavior of the zinc blende $\left(\mathrm{Zn}_{1-\mathrm{x}} \mathrm{Fe}_{\mathrm{x}}\right) \mathrm{S}$ solid solution rather corresponds to the two-mode model: As the Fe content changes, the Raman spectra change correspondingly, first continuously and then stepwise, starting from $x=0.1$ [61]. The behavior 
of the $(\mathrm{Zn}, \mathrm{Cd}, \mathrm{Fe})(\mathrm{S}, \mathrm{O}, \mathrm{Se})$ complex solid solution in our case fits neither of the two previous models but is rather multi-modal. This is evident in three strong modes that appeared at increasing $\mathrm{x}$.

The vibration states of the $\mathrm{ZnS}_{\mathrm{cub}}, \mathrm{FeS}_{\mathrm{cub}}$, and $\mathrm{CdS}_{\mathrm{cub}}$ endmembers of the $\mathrm{Zn}-\mathrm{Fe}-\mathrm{Cd}$ solid solution were calculated with the LADY software for lattice-dynamic simulations. According to the simulations, strong LO bands should appear in the first-order Raman spectra: $350 \mathrm{~cm}^{-1}$ for cubic ZnS (sphalerite), $359 \mathrm{~cm}^{-1}$ for cubic FeS (rudashevskite), and $324 \mathrm{~cm}^{-1}$ for cubic CdS (hawleyite). However, the observed spectrum misses two latter bands, which proves the absence of $\mathrm{FeS}_{\mathrm{cub}}$ and $\mathrm{CdS}_{\text {cub }}$ discrete phases exsolved from the parent homogeneous solid solution, as well as a statistical distribution of $\mathrm{Zn}, \mathrm{Fe}$, and $\mathrm{Cd}$ cations at the same positions of the mineral structure.

The homogeneity of the studied crystals revealed by the spatial micro-Raman analysis characterizes the solid solution as regular. Judging by the high content of oxygen in the sample, it may contain $\mathrm{Zn}$ and O-bearing microscopic inclusions or layers/films in the $\mathrm{ZnS}$ matrix. Zinc oxide $(\mathrm{ZnO})$ can exist either as hexagonal zincite (würtzite type) or cubic (zinc blende-type) modifications. The Raman spectra of zincite should include additionally a sharp band at about $437 \mathrm{~cm}^{-1}$ (TO) and another wide band at $524 \mathrm{~cm}^{-1}$ (LO) with a $562-\mathrm{cm}^{-1}$ shoulder (RRUFF, R060027 card); in the case of $\mathrm{ZnO}_{\mathrm{cub}}$, additional bands may appear at about 340 (TO) and $525 \mathrm{~cm}^{-1}$ (LO) [122,123]. However, none of these bands were observed in our sample. The vibration modes of the cubic modifications are known [122] to have the same frequencies as those of the hexagonal phase and generally cannot be used for discrimination between the two structures. Therefore, our data suggest that $\mathrm{O}^{2-}$ may substitute for sulfur in regular sites of the $\mathrm{ZnS}$ cubic cell, and its position is averaged.

Thus, single-crystal XRD and Raman spectroscopy concordantly indicate that the natural crystal of the complex composition $(\mathrm{Zn}, \mathrm{Cd}, \mathrm{Fe}))(\mathrm{S}, \mathrm{O}, \mathrm{Se})$ is isostructural with sphalerite and has a disordered distribution of all main impurities in the $\mathrm{ZnS}_{\text {cub }}$ lattice. This appears to be the first proof that a restricted $\mathrm{ZnS}_{1-\mathrm{x}} \mathrm{O}_{\mathrm{x}}$ solid solution can form in nature.

\subsection{Oxygen in ZnS Compounds: Experimental Background and Crystal-Growth Practice}

Among the VIB subgroup chalcogens, oxygen most strongly differs from S, Se, and Te, which it replaces; the incorporation of even minor amounts of $\mathrm{O}$ can cause significant local changes to the lattice of chalcogenides and to the properties of their composites. Chalcogens differ from oxygen in greater Pauling electronegativity (3.44 against 2.58 for S, 2.55 for Se, and 2.1 for Te) and lower polarizability $\left(3.88 \times 10^{-24} \mathrm{~cm}^{3}\right.$ for $\mathrm{O}^{2-}$ against $10.2 \cdot \times 10^{-24} \mathrm{~cm}^{3}$ for $\mathrm{S}^{2-}, 10.5 \cdot \times 10^{-24} \mathrm{~cm}^{3}$ for $\mathrm{Se}^{2-}$, and $14.0 \times 10^{-24} \mathrm{~cm}^{3}$ for $\mathrm{Te}^{2-}$ ) [66]. This dissimilarity entrains differences in the crystal chemistry of oxides and chalcogenides for nearly all element combinations and restricts isomorphic substitutions between $\mathrm{S}^{2-}$ and $\mathrm{O}^{2-}$. Nevertheless, it is well known that limited amounts of oxygen can substitute for isovalent $\mathrm{S}^{2-}$ in chalcogenides [66].

The possibility of $\mathrm{O}$ incorporation into sulfides was checked for the first time by Skinner and Barton [58], who synthesized oxygen-substituted sphalerite and würtzite (with $0.7 \mathrm{wt} \%$ and $1.0 \mathrm{wt} \%$ $\mathrm{ZnO}$, respectively). However, the $\mathrm{O}^{2-} \rightarrow \mathrm{S}^{2-}$ substitution in natural minerals is hard to prove unambiguously even nowadays. In the case of $\mathrm{ZnS}$, this is especially difficult because $\mathrm{ZnS}$ hex and $\mathrm{ZnO}$ belong to the same structure type (würtzite) and share similarity in the parameters of flat nets in the two phases, which favors their epitaxial co-crystallization. This feature has been employed in the practice of industrial synthesis of semiconductors as the growth of a 'layered cake' consisting of alternated nanometer layers of $\mathrm{ZnS}_{\text {hex }}$ and $\mathrm{ZnO}$ that have different conductivity types [27].

The ternary semiconductor alloys of II-VI groups, with their large energy band gap and binding energy of excitons, are of growing interest for uses in optoelectric devices operating in the visible and ultraviolet spectral regions. They are specifically $\mathrm{ZnS}, \mathrm{ZnO}$, and their dilute ternary alloys $\mathrm{ZnS}_{1-\mathrm{x}} \mathrm{O}_{\mathrm{x}}$ having semiconducting properties [124]. The diverse applications of $\mathrm{ZnS}$ crystals and films in various high-tech devices (solar cells, electroluminescent diodes, dielectric filters, etc.) induced investigations into the interactions of $\mathrm{ZnS}$ (both sphalerite and würtzite) with oxygen during the synthesis and oxidation of ZnS composites exposed to air. The studies revealed three main 
modes of oxygen occurrence in $\mathrm{ZnS}$ phases: (i) restricted solid solutions $\mathrm{ZnS}_{1-\mathrm{x}} \mathrm{O}_{\mathrm{x}}$ (lattice oxygen); (ii) heterophase CVD films (combination of $\mathrm{ZnS}, \mathrm{ZnO}$, and $\mathrm{Zn}(\mathrm{OH})_{2}$ films and $\mathrm{Zn}$ sulfates); (iii) surface oxidation of $\mathrm{ZnS}$ during annealing or operation which produces $\mathrm{Zn}$ sulfate films $[28,120,125]$.

It appears pertinent to compare the scale of $\mathrm{O}^{2-} \rightarrow \mathrm{S}^{2-}$ substitutions in synthetic and natural $\mathrm{CM}$ sphalerites, because our single-crystal XRD and micro-Raman spectroscopy results revealed no extraneous phases in the CM sphalerite crystals but proved the presence of lattice-bound oxygen.

The incorporation of oxygen atoms into the $\mathrm{ZnS}$ structure leads to dramatic changes of the band pattern and related fundamental properties, which makes the alloys technologically important $[28,120]$. Much effort was put into constraining the stability fields of the phases where sustainable synthesis of such crystals is possible: $\mathrm{ZnO}$ doped with $\mathrm{S}^{2-}$ and $\mathrm{ZnS}$ doped with $\mathrm{O}^{2-}$. There is a large miscibility gap between $\mathrm{ZnO}$ (würtzite type) and $\mathrm{ZnS}$ (sphalerite type) due to their structural difference at temperatures below $1020^{\circ} \mathrm{C}$ and to the considerable difference of the $\mathrm{S}^{2-}$ and $\mathrm{O}^{2-}$ radii (1.73 and $1.40 \AA$, respectively). The $\mathrm{ZnO}-\mathrm{ZnS}$ system exhibits an approximately symmetrical region of immiscibility between 5 and $96 \mathrm{~mol} \% \mathrm{ZnS}$ at $900{ }^{\circ} \mathrm{C}$. XRD of single crystals and polycrystalline samples indicates that $\mathrm{ZnO}_{1-\mathrm{x}} \mathrm{S}_{\mathrm{x}}$ and $\mathrm{ZnS}_{1-\mathrm{x}} \mathrm{O}_{\mathrm{x}}$ phases with $x$ between 0.0 and 0.05 are thermodynamically stable at $900{ }^{\circ} \mathrm{C}$ [26]. The $\mathrm{ZnS}_{1-\mathrm{x}} \mathrm{O}_{\mathrm{x}}$ zinc blende crystals with an oxygen content of $x=0.03125$ to 0.0625 were synthesized by Belouche et al. [28]. The thermodynamic stability and electronic properties of würtzite and zinc blende $\mathrm{ZnS}_{1-x} \mathrm{O}_{x}$ phases were also studied theoretically with first-principle simulations [27] and a composition-temperature $(x-\mathrm{T})$ diagram was plotted for the Zn-S-O system. The results show that the formation enthalpy of würtzite and sphalerite structure types of $\mathrm{ZnS}_{1-\mathrm{x}} \mathrm{O}_{\mathrm{x}}$ alloys with randomly distributed $\mathrm{O}$ atoms increases steeply as a function of $x$. This produces spinodal curves for both $\mathrm{ZnS}_{1-\mathrm{x}} \mathrm{O}_{\mathrm{x}}$ structural types and limits the stability fields to the compositions near the $\mathrm{ZnS}$ and $\mathrm{ZnO}$ endmembers (at $x<0.1$ ). For example, the solubility limit was less than $2 \%$ at a growth temperature of $1000 \mathrm{~K}$ [27].

Oxygen in the structure of stoichiometric sphalerite commonly occurs as solitary atoms. It is statistically distributed in lattice nodes and is interpreted as dissolved [ $\left.\mathrm{O}_{\mathrm{S}}\right]$. $\mathrm{O}_{\mathrm{S}}$ close to the limit concentration coexists with excess $\mathrm{Zn}$ in the $\mathrm{ZnS}$ crystals, where excess $\mathrm{Zn}$ can exist as a donor in oxygenated SA complexes [120]. The concentration of $\left[\mathrm{O}_{S}\right]$ in synthetic sphalerite reaches $\approx 1.3 \mathrm{wt} \%$ near the temperature of the $\mathrm{ZnS}_{\text {cub }} \rightarrow \mathrm{ZnS}_{\text {hex }}$ transition $\left(1020-1040{ }^{\circ} \mathrm{C}\right)$. In the region of homogeneous solid solutions $\mathrm{ZnS}_{1-\mathrm{x}} \mathrm{O}_{\mathrm{x}}$, the range of dissolved oxygen $\left(\mathrm{O}_{\mathrm{S}}\right)$ contents spans about three orders of magnitude: from $(2-3) \times 10^{20} \mathrm{~cm}^{-3}$ in compounds with $\mathrm{Zn}$ excess relative to the stoichiometric $\mathrm{ZnS}$ composition, at the temperature of 940 to $1060{ }^{\circ} \mathrm{C}$. Without additional doping, $\mathrm{O}_{S}$ is at a level of $10^{17}-10^{18} \mathrm{~cm}^{-3}$ in $\mathrm{ZnS}$ crystals and about $10^{19}-10^{20} \mathrm{~cm}^{-3}$ in polycrystalline ZnS compounds [120].

The amount of lattice-bound and randomly distributed oxygen revealed by single-crystal XRD in high-temperature $\left(\sim 800-850{ }^{\circ} \mathrm{C}\right)$ natural sphalerite of the $\mathrm{Zn}_{0.96} \mathrm{Cd}_{0.02} \mathrm{Fe}_{0.02}\left(\mathrm{~S}_{0.946(8)} \mathrm{O}_{0.046(8)} \mathrm{Se}_{0.007(4)}\right)$ composition is in the range of $0<x \leq 0.05$ inferred for $\mathrm{ZnS}_{1-\mathrm{x}} \mathrm{O}_{\mathrm{x}}$ single crystals stable at $\mathrm{T} \approx 900{ }^{\circ} \mathrm{C}$.

\section{Conclusions}

1. Sphalerite from the Tulul al Hammam area in central Jordan is a chief sulfide mineral in combustion metamorphic (CM) marbles that formed after the Maastrichtian-Paleocene bioproductive 'oil shales' with abundant authigenic ZnS mineralization: würtzite and sphalerite highly enriched in $\mathrm{Cd}, \mathrm{Cu}, \mathrm{As}, \mathrm{Ga}, \mathrm{Ge}, \mathrm{Ag}$, and $\mathrm{Sb}$ and less-abundant pyrite bearing $\mathrm{Ni}, \mathrm{Cu}$, As, Se, $\mathrm{Mo}$, and $\mathrm{Ag}$. The precursor chalky sediments were annealed by spontaneous combustion of disseminated S-rich organic matter at the parameters of sanidinite facies metamorphism: $\sim 800-850^{\circ} \mathrm{C}$, ambient pressure, and a moderately reduced environment.

2. High-temperature annealing of the sediments had several consequences: Recrystallization of authigenic würtzite and sphalerite; total consumption of pyrite; and compositional and textural homogenization of CM sphalerite, whereby the latter acquired a trace-element composition less diverse than in the precursor $\mathrm{ZnS}$. Sphalerites from particular geological environments, such as CM marbles, belong to a single generation; they became compositionally equilibrated and were quenched 
near the peak temperature of combustion metamorphism. The Se-rich, and Ni- and Cu-bearing heterogeneous mineral assemblages rarely found in these rocks may, to a high probability, record the primary heterogeneous distribution of TE hosts in the sedimentary protolith.

3. In spite of high formation temperatures, the analyzed CM sphalerites have low Fe but high Cd contents $\left(X_{a v}=1.4 \%, X_{\max }=3.7 \mathrm{wt} \%\right.$ and $X_{a v} .=2.0 \mathrm{wt} \%, X_{\max }=14.0 \mathrm{wt} \%$, respectively) concordantly with the Muwaqqar Fm. marly chalk, which are Fe-poor but rich in $\mathrm{Zn}$ and $\mathrm{Cd}$. This effect becomes still more prominent due to iron oxidation during the annealing of sediments. The greatest part of iron in the marbles occurs as $\mathrm{Fe}^{+3}$ hosted by $\mathrm{Ca}$ ferrites or aluminate ferrites. The $\mathrm{CM}$ sphalerites also inherited Se $\left(X_{a v}=1.5 \%, X_{\max }=6.8 \mathrm{wt} \% \mathrm{Se}\right)$ from the precursor sediments. Another feature is that they have low and relatively stable concentrations of $\mathrm{Cu}, \mathrm{Ga}, \mathrm{Ag}$, In, $\mathrm{Sn}$, and $\mathrm{Sb}$ while the $\mathrm{Ge}, \mathrm{As}, \mathrm{Mo}, \mathrm{Te}, \mathrm{Tl}, \mathrm{Bi}, \mathrm{Pb}$, and $\mathrm{Au}$ contents are below detection limits. These signatures are not universal for all high-temperature low-pressure metamorphic sphalerites but instead represent a local geological setting.

4. Under the CM conditions (one-stage high-temperature dry annealing and rapid quenching), which are very similar to the experimental conditions of pyrosynthesis, fractionation of trace elements into sphalerite is mainly controlled by crystal structures. This fractionation is limited, though continuous or restricted solid solutions exist in the Zn-Me-S-Se systems ( $\mathrm{Me}=\mathrm{Fe}, \mathrm{Cd}, \mathrm{Ni}, \mathrm{Co}, \mathrm{Cu}, \mathrm{Ga}$, and $\mathrm{In}$ ) at high temperatures. At the same time, $\mathrm{Fe}, \mathrm{Cd}, \mathrm{Se}, \mathrm{Ni}$, and $\mathrm{Cu}$ also form their own phases in the CM marbles.

5. Single-crystal XRD and Raman spectroscopy data provide consistent evidence that natural crystals of the $(\mathrm{Zn}, \mathrm{Cd}, \mathrm{Fe}))(\mathrm{S}, \mathrm{O}, \mathrm{Se})$ composition are isostructural to sphalerite and have disordered distributions of all main impurities, which are lattice bound. This appears to be the first proven case of a $\mathrm{ZnO}-\mathrm{ZnS}$ restricted solid solution that has formed in nature within the stability field corresponding to a $\mathrm{ZnS}_{1-\mathrm{x}} \mathrm{O}_{\mathrm{x}}(0<\mathrm{x} \leq 0.05)$ homogeneous compound at $900{ }^{\circ} \mathrm{C}$.

6. As we found out, sulfides and oxychalcogenides can be used for petrogenetic interpretation as sensitive and independent tracers of CM conditions (temperature, redox regime) and metal sources, at least for quickly quenched anhydrous sanidinite-facies rocks.

Author Contributions: Project idea: E.V.S., S.N.K. and Y.V.S.; Mineralogy and petrography: E.V.S., S.N.K., V.V.S., H.N.K. and A.S.D.; Field work: H.N.K., E.V.S. and S.N.K; Geochemistry: E.V.S., H.N.K. and S.N.K.; SEM analyses: E.V.S., V.V.S, N.S.K. and V.A.D.; EPMA analyses: E.V.S., V.V.S. and V.A.D.; LA-ICPMS analysis: E.V.S. and D.A.A.; XRD analysis, and preconditioning of samples, and interpretation: Y.V.S.; Raman spectroscopy and interpretation: S.V.G.; Interpretation of analytical data: E.V.S., S.N.K., Y.V.S, V.V.S., H.N.K. and A.S.D.; Visualization: S.N.K., A.S.D. and H.N.K.; Writing: E.V.S., S.N.K.,Y.V.S. and S.V.G. All authors have read and agreed to the published version of the manuscript.

Funding: This research was funded by the Russian Science Foundation, grant number 17-17-01056P.

Acknowledgments: The manuscript benefited much from the thoughtful review and valuable comments of anonymous reviewers, which we accepted with gratitude. We wish to thank E. Nigmatulina, and M. Khlestov who performed SEM and EPMA analytical works (IGM, Novosibirsk) and to T. Perepelova for helpful advice. Our colleagues Wadah Faris, Yousef Abu Salheh, and Azzam Azzarah (University of Jordan, Amman) are acknowledged for their help in the field work. The study was supported by grant 17-17-01056P from the Russian Science Foundation.

Conflicts of Interest: The authors declare no conflict of interest.

\section{References}

1. Cook, N.J.; Ciobanu, C.L.; Pring, A.; Skinner, W.; Shimizu, M.; Danyushevsky, L.; Saini-Eidukat, B. Trace and minor elements in sphalerite: A LA-ICPMS study. Geochim. Cosmochim. Acta 2009, 73, 4761-4791. [CrossRef]

2. Barton, P.B. Sulfide Petrology; Ribbe, P.H., Ed.; Mineralogical Society of America Reviews in Mineralogy: Sully, VA, USA, 1974; Volume 1, pp. B1-B11.

3. Brown, J.L.; Christy, A.G.; Ellis, D.J.; Arculus, R.J. Prograde sulfide metamorphism in blueschist and eclogite, New Caledonia. J. Petrol. 2014, 55, 643-670. [CrossRef] 
4. Fleurance, S.; Cuney, M.; Malartre, M.; Reyx, J. Origin of the extreme polymetallic enrichment (Cd, Cr, Mo, $\mathrm{Ni}, \mathrm{U}, \mathrm{V}, \mathrm{Zn}$ ) of the Late Cretaceous-Early Tertiary Belqa Group, central Jordan. Palaeogeog. Palaeoclim. Palaeoecol. 2013, 369, 201-219. [CrossRef]

5. Sharygin, V.V.; Lazic, B.; Armbruster, T.M.; Murashko, M.N.; Wirth, R.; Galuskina, I.O.; Galuskin, E.V.; Vapnik, Y.; Britvin, S.N.; Logvinova, A.M. Shulamitite $\mathrm{Ca}_{3} \mathrm{TiFe}^{3+} \mathrm{AlO}_{8}-\mathrm{A}$ new perovskite-related mineral from Hatrurim Basin, Israel. Eur. J. Miner. 2013, 25, 97-111. [CrossRef]

6. Sharygin, V.V. Orthorhombic $\mathrm{CaCr}_{2} \mathrm{O}_{4}$ in Phosphide-Bearing Gehlenite-Rankinite Paralava from Hatrurim Basin, Israel. In Proceedings of the Conference Magmatism of the Earth and Related Strategic Metal Deposits, Saint Petersburg, Russia, 23-26 May 2019; pp. 272-276.

7. Britvin, S.N.; Murashko, M.N.; Vapnik, Y.; Polekhovsky, Y.S.; Krivovichev, S.V. Earth's phosphides in Levant and insights into the source of Archean prebiotic phosphorus. Sci. Rep. 2015, 5, 8355. [CrossRef]

8. Britvin, S.N.; Murashko, M.N.; Vereshchagin, O.S.; Vapnik, Y.; Shilovskikh, V.V.; Vlasenko, N.S. Polekhovskyite, IMA 2018-147. CNMNC Newsletter No. 48, April 2019: Page 316. Miner. Mag. 2019, 83, 315-317.

9. Britvin, S.N.; Murashko, M.; Vapnik, Y.; Polekhovsky, Y.S.; Krivovichev, S.V.; Vereshchagin, O.S.; Shilovskikh, V.V.; Krzhizhanovskaya, M.G. Negevite, the pyrite-type $\mathrm{NiP}_{2}$, a new terrestrial phosphide. Am. Miner. 2020, 105, 422-427. [CrossRef]

10. Britvin, S.N.; Murashko, M.N.; Vapnik, Y.; Polekhovsky, Y.S.; Krivovichev, S.V.; Krzhizhanovskaya, M.O.; Vereshchagin, O.S.; Shilovskikh, V.V.; Vlasenko, N.S. Transjordanite, $\mathrm{Ni}_{2} \mathrm{P}$, a new terrestrial and meteoritic phosphide, and natural solid solutions barringerite-transjordanite (hexagonal $\mathrm{Fe}_{2} \mathrm{P}-\mathrm{Ni} \mathrm{i}_{2} \mathrm{P}$ ). Am. Miner. 2020, 105, 428-436. [CrossRef]

11. Britvin, S.N.; Murashko, M.N.; Vapnik, Y.; Polekhovsky, Y.S.; Krivovichev, S.V.; Vereshchagin, O.S.; Shilovskikh, V.V.; Vlasenko, N.S.; Krzhizhanovskaya, M.G. Halamishite, $\mathrm{Ni}_{5} \mathrm{P}_{4}$, a new terrestrial phosphide in the Ni-P system. Phys. Chem. Miner. 2020, 47, 3. [CrossRef]

12. Galuskina, I.O.; Krüger, B.; Galuskin, E.V.; Krüger, H.; Vapnik, Y.; Murashko, M.; Agakhanov, A.A.; Pauluhn, A.; Olieric, V. Zoharite, IMA 2017-049. CNMNC Newsletter No. 39. Miner. Mag. 2017, 81, 1279-1286.

13. Galuskina, I.O.; Krüger, B.; Galuskin, E.V.; Krüger, H.; Vapnik, Y.; Banasik, K.; Murashko, M.; Agakhanov, A.A.; Pauluhn, A. Gmalimite, IMA 2019-007. CNMNC Newsletter No. 50. Miner. Mag. 2019, 83, 31.

14. Sokol, E.V.; Kozmenko, O.A.; Khoury, H.N.; Kokh, S.N.; Novikova, S.A.; Nefedov, A.A.; Sokol, I.A.; Zaikin, P. Calcareous sediments of the Muwaqqar Chalk Marl Formation, Jordan: Mineralogical and geochemical evidences for Zn and Cd enrichment. Gondwana Res. 2017, 46, 204-226. [CrossRef]

15. Sokol, E.V.; Kokh, S.N.; Sharygin, V.V.; Danilovsky, V.A.; Seryotkin, Y.V.; Liferovich, R.; Deviatiiarova, A.S.; Nigmatulina, E.N.; Karmanov, N.S. Mineralogical diversity of $\mathrm{Ca}_{2} \mathrm{SiO}_{4}$-bearing combustion metamorphic rocks in the Hatrurim Basin: Implications for storage and partitioning of elements in oil shale clinkering. Minerals 2019, 9, 465. [CrossRef]

16. Danilovsky, V.A.; Deviatiiarova, A.S. Unusual sulfides Fe, K, Ca, Ni, Zn, Ag and selenides Fe, Cu, Ni, $\mathrm{Zn}$, Cd from combustion metamorphic spurrite marbles, the Hatrurim Formation. In Proceedings of the Conference Mineralogical Museum 2019. Mineralogy Yesterday, Today, Tomorrow, Saint Petersburg, Russia, 17-19 September 2019; pp. 110-112.

17. Techer, I.; Khoury, H.N.; Salameh, E.; Rassineux, F.; Claude, C.; Clauer, N.; Pagel, M.; Lancelot, J.; Hamelin, B.; Jacquot, E. Propagation of high-alkaline fluids in an argillaceous formation: Case study of the Khushaym Matruk natural analogue (Central Jordan). J. Geochem. Explor. 2006, 90, 53-67. [CrossRef]

18. Khoury, H.; Sokol, E.; Clark, I. Calcium uranium oxides from Central Jordan: Mineral assemblages, chemistry, and alteration products. Can. Miner. 2015, 53, 61-82. [CrossRef]

19. Khoury, H.N.; Sokol, E.V.; Kokh, S.N.; Seryotkin, Y.V.; Kozmenko, O.A.; Goryainov, S.V.; Clark, I.D. Intermediate members of the lime-monteponite solid solutions $\left(\mathrm{Ca}_{1-\mathrm{x}} \mathrm{Cd}_{\mathrm{x}} \mathrm{O}, \mathrm{x}=0.36-0.55\right)$ : Discovery in natural occurrence. Am. Miner. 2016, 101, 146-161. [CrossRef]

20. Khoury, H.N.; Sokol, E.V.; Kokh, S.N.; Seryotkin, Y.V.; Nigmatulina, E.N.; Goryainov, S.V.; Belogub, E.V.; Clark, I.D. Tululite, $\mathrm{Ca}_{14}\left(\mathrm{Fe}^{3+}, \mathrm{Al}\right)\left(\mathrm{Al}, \mathrm{Zn}, \mathrm{Fe}^{3+}, \mathrm{Si}, \mathrm{P}, \mathrm{Mn}, \mathrm{Mg}\right){ }_{15} \mathrm{O}_{36}$ : A new Ca zincate-aluminate from combustion metamorphic marbles, Central Jordan. Miner. Petrol. 2016, 110, 125-140. [CrossRef] 
21. Khoury, H.; Kokh, S.N.; Sokol, E.V.; Likhacheva, A.Y.; Seryotkin, Y.V.; Belogub, E.V. Ba- and Sr-mineralization of fossil fish bones from metamorphosed Belqa Group sediments, Central Jordan: An integrated methodology. Arab. J. Geosci. 2016, 9, 461. [CrossRef]

22. Sokol, E.V.; Kokh, S.N.; Khoury, H.N.; Seryotkin, Y.V.; Goryainov, S.V. Long-term immobilization of $\mathrm{Cd}^{2+}$ at the Tulul al Hammam natural analogue site, Central Jordan. Appl. Geochem. 2016, 70, 43-60. [CrossRef]

23. Sokol, E.V.; Kokh, S.N.; Khoury, H.N.; Seryotkin, Y.V.; Goryainov, S.V.; Novikova, S.A.; Sokol, I.A. Natural analogue approaches to prediction of long-term behavior of $\mathrm{Ca}_{2} \mathrm{UO}_{5} \cdot 2-3 \mathrm{H}_{2} \mathrm{O}$ 'X-Phase': Case study from Tulul al Hammam site, Jordan. Arab. J. Geosci. 2017, 10, 512. [CrossRef]

24. Vapnik, Y.; Galuskin, E.V.; Galuskina, I.O.; Kusz, J.; Stasiak, M.; Krzykawski, T.; Dulski, M. Qatranaite, $\mathrm{CaZn}_{2}(\mathrm{OH})_{6} \cdot 2 \mathrm{H}_{2} \mathrm{O}$ : A new mineral from altered pyrometamorphic rocks of the Hatrurim Complex, Daba-Siwaqa, Jordan. Eur. J. Miner. 2019, 31, 575-584. [CrossRef]

25. Hotje, U.; Rose, C.; Binnewies, M. Lattice constants and molar volume in the system ZnS, ZnSe, CdS, CdSe. Solid State Sci. 2003, 5, 1259-1262. [CrossRef]

26. Locmelis, S.; Brünig, C.; Binnewies, M.; Börger, A.; Becker, K.D.; Homann, T.; Bredow, T. Optical band gap in the system $\mathrm{ZnO}_{1-x} \mathrm{~S}_{x}$. An experimental and quantum chemical study. J. Mater. Sci. 2007, 42, 1965-1971. [CrossRef]

27. Fan, X.F.; Shen, Z.X.; Lu, Y.M.; Kuo, J.L. A theoretical study of thermal stability and electronic properties of würtzite and zinc blende $\mathrm{ZnO}_{\mathrm{x}} \mathrm{S}_{1-\mathrm{x}}$. New J. Phys. 2009, 11, 093008. [CrossRef]

28. Bellouche, A.; Gueddim, A.; Zerroug, S.; Bouarissa, N. Elastic properties and optical spectra of $\mathrm{ZnS}_{1-\mathrm{x}} \mathrm{O}_{\mathrm{x}}$ dilute semiconductor alloys. Optik 2016, 127, 11374-11378. [CrossRef]

29. Zagorac, D.; Zagorac, J.; Schön, J.C.; Stojanović, N.; Matović, B. ZnO/ZnS (hetero) structures: Ab initio investigations of polytypic behavior of mixed $\mathrm{ZnO}$ and $\mathrm{ZnS}$ compounds. Acta Cryst. B Struct. Sci. Cryst. Eng. Mater. 2018, B74, 628-642. [CrossRef]

30. Abed, A.M.; Arouri, K.R.; Boreham, C.J. Source rock potential of the phosphorite-bituminous chalk-marl sequence in Jordan. Mar. Pet. Geol. 2005, 22, 413-425. [CrossRef]

31. Powell, J.H.; Moh'd, B.K. Evolution of Cretaceous to Eocene alluvial and carbonate platform sequences in central and south Jordan. GeoArabia 2011, 16, $29-82$.

32. Khoury, H.; Salameh, E.; Clark, I. Mineralogy and origin of surficial uranium deposits hosted in travertine and calcrete from central Jordan. Appl. Geochem. 2014, 43, 49-65. [CrossRef]

33. Shatsky, V.; Sitnikova, E.; Kozmenko, O.; Palessky, S.; Nikolaeva, I.; Zayachkovsky, A. Behavior of incompatible elements during ultrahigh-pressure metamorphism (by the example of rocks of the Kokchetav massif). Russ. Geol. Geophys. 2006, 47, 482-496. [CrossRef]

34. Element, C.A.S. Method 3051A-microwave assisted acid digestion of sediments, sludges, soils, and oils. Z. Anal. Chem. 2007, 111, 362-366.

35. Carvalho, L.; Monteiro, R.; Figueira, P.; Mieiro, C.; Almeida, J.; Pereira, E.; Magalhães, V.; Pinheiro, L.; Vale, C. Vertical distribution of major, minor and trace elements in sediments from mud volcanoes of the Gulf of Cadiz: Evidence of Cd, As and Ba fronts in upper layers. Deep Sea Res. Part I Oceanogr. Res. Pap. 2018, 131, 133-143. [CrossRef]

36. Shuvaeva, O.V.; Gustaytis, M.A.; Anoshin, G.N. Mercury speciation in environmental solid samples using thermal release technique with atomic absorption detection. Anal. Chim. Acta 2008, 621, 148-154. [CrossRef]

37. Lavrent'ev, Y.G.; Karmanov, N.S.; Usova, L.V. Electron probe microanalyses of minerals: Microanalyzer or scanning electron microscope? Russ. Geol. Geophys. 2015, 56, 1154-1161. [CrossRef]

38. Sharygin, V.V.; Yakovlev, G.A.; Wirth, R.; Seryotkin, Y.V.; Sokol, E.V.; Nigmatulina, E.N.; Karmanov, N.S.; Pautov, L.A. Nataliakulikite, $\mathrm{Ca}_{4} \mathrm{Ti}_{2}\left(\mathrm{Fe}^{3+}, \mathrm{Fe}^{2+}\right)\left(\mathrm{Si}_{,} \mathrm{Fe}^{3+}, \mathrm{Al}^{3} \mathrm{O}_{11}\right.$, a new perovskite-supergroup mineral from Hatrurim Basin, Negev Desert, Israel. Minerals 2019, 9, 700. [CrossRef]

39. Sharygin, V.V. Phase $\mathrm{CuCrS}_{2}$ in Iron Meteorite Uakit (IIAB), Buryatia, Russia: Preliminary Data; Votyakov, S., Kiseleva, D., Grokhovsky, V., Shchapova, Y., Eds.; Earth and Environmental Sciences Book Series, Minerals: Structure, Properties, Methods of Investigation; Springer: Berlin/Heidelberg, Germany, 2020; pp. 229-236. [CrossRef]

40. Goldstein, J.I.; Newbury, D.E.; Echlin, P.; Joy, D.C.; Lyman, C.E.; Lifshin, E.; Sawyer, L.; Michael, J.R. Quantitative X-ray Analysis: The Basics. In Scanning Electron Microscopy and X-ray Microanalysis; Springer: Berlin/Heidelberg, Germany, 2003; pp. 391-451. 
41. Humphries, D.W. The Preparation of Thin Sections of Rocks, Minerals and Ceramics. In Royal Microscopical Society Microscopy Handbooks (Book 24); Oxford University Press: Oxford, UK, 1992.

42. Artemyev, D.A.; Ankushev, M.N. Trace elements of $\mathrm{Cu}-(\mathrm{Fe})$-sulfide inclusions in bronze age copper slags from South Urals and Kazakhstan: Ore sources and alloying additions. Minerals 2019, 9, 746. [CrossRef]

43. Longerich, H.P.; Jackson, S.E.; Günther, D. Inter-laboratory note. Laser ablation inductively coupled plasma mass spectrometric transient signal data acquisition and analyte concentration calculation. J. Anal. At. Spectrom. 1996, 11, 899-904. [CrossRef]

44. Wilson, S.A.; Ridley, W.I.; Koenig, A.E. Development of sulphide calibration standards for the laser ablation inductively-coupled plasma mass spectrometry technique. J. Anal. At. Spectrom. 2002, 17, 406-409. [CrossRef]

45. Paton, C.; Hellstrom, J.; Paul, B.; Woodhead, J.; Hergt, J. Iolite: Freeware for the visualisation and processing of mass spectrometric data. J. Anal. At. Spectrom. 2011, 26, 2508-2518. [CrossRef]

46. CrysAlis, C.C.D. CrysAlis RED 171.37.35; Oxford Diffraction Ltd.: Abingdon, UK, 2008.

47. Model S506 Interactive Peak Fit User's Manual; Canberra Industries Inc.: Canberra, Australia, 2002.

48. Abed, A.M.; Arouri, K.; Amiereh, B.S.; Al-Hawari, Z. Characterization and genesis of some Jordanian oil shales. Dirasat Pure Sci. 2009, 36, 7-17.

49. Abed, A. Review of uranium in the Jordanian phosphorites: Distribution, genesis and industry. Jordan J. Earth Environ. Sci. 2012, 4, 35-45.

50. Abed, A.; Sadaqah, R. Enrichment of uranium in the uppermost Al-Hisa Phosphorite Formation, Eshidiyya basin, southern Jordan. J. Afr. Earth Sci. 2013, 77, 31-40. [CrossRef]

51. März, C.; Wagner, T.S.; Al-Alaween, A.M.; Boorn, S.; Podlaha, O.G.; Kolonic, S.; Poulton, S.W.; Schnetger, B.; Brumsack, H.-J. Repeated enrichment of trace metals and organic carbon on an Eocene high-energy shelf caused by anoxia and reworking. Geology 2016, 44, 1011-1014. [CrossRef]

52. Hakimi, M.H.; Abdullah, W.H.; Alqudah, M.; Makeen, Y.M.; Mustapha, K.A. Organic geochemical and petrographic characteristics of the oil shales in the Lajjun area, Central Jordan: Origin of organic matter input and preservation conditions. Fuel 2016, 181, 34-45. [CrossRef]

53. Hamarneh, Y. Oil Shale Resources Development in Jordan; Natural Resources Authority: Amman, Jordan, 1998; p. 98.

54. Khoury, H. Tripolization of chert in Jordan. Sediment Geol. 1987, 53, 305-310. [CrossRef]

55. Khoury, H. Mineralogy and petrography of some opaline phase from Jordan. Neues Jahrb. Miner. Abh. 1989, 10, 433-440.

56. Skinner, B.J. Unit-cell edges of natural and synthetic sphalerites. Am. Miner. 1961, 46, 1399-1411.

57. Sheldrick, G.M. SHELXT-Integrated space-group and crystal-structure determination. Acta Crystallogr. A Found. Adv. 2015, 71, 3-8. [CrossRef]

58. Skinner, B.J.; Barton, P.B. The substitution of oxygen for sulfur in würtzite and sphalerite. Am. Miner. 1960, 45, 612-625.

59. Van Aswegen, J.T.S.; Verleger, H. Röntgenographische untersuchung des systems ZnS-FeS. Naturwiss 1960, 47, 131. [CrossRef]

60. Barton, P.B.; Toulmin, P. Phase relation involving sphalerite in the Fe-Zn-S system. Econ. Geol. 1966, 61, 815-849. [CrossRef]

61. Osadchii, E.G.; Gorbaty, Y.E. Raman spectra and unit cell parameters of sphalerite solid solutions $\left(\mathrm{Fe}_{\mathrm{x}} \mathrm{Zn}_{1-\mathrm{x}} \mathrm{S}\right)$. Geochim. Cosmochim. Acta 2010, 74, 568-573. [CrossRef]

62. Brese, N.E.; O'Keeffe, M. Bond-valence parameters for solids. Acta Crystallogr. B Struct. Sci. 1991, 47, $192-197$. [CrossRef]

63. Serrano, J.; Cantarero, A.; Cardona, M.; Garro, N.; Lauck, R.; Tallman, R.E.; Ritter, T.M.; Weinstein, B.A. Raman scattering in $\beta$-ZnS. Phys. Rev. B. 2004, 69, 014301. [CrossRef]

64. Fairbrother, A.; Izquierdo-Roca, V.; Fontané, X.; Ibáñez, M.; Cabot, A.; Saucedo, E.; Pérez-Rodríguez, A. ZnS grain size effects on near-resonant Raman scattering: Optical non-destructive grain size estimation. CrystEngComm 2014, 16, 4120-4125. [CrossRef]

65. Wright, K.; Gale, J.D. Interatomic potentials for the simulation of the zinc-blende and würtzite forms of ZnS and CdS: Bulk structure, properties, and phase stability. Phys. Rev. B Condens. Matter. Mater. Phys. 2004, 70, 035211. [CrossRef] 
66. Makovicky, E. Crystal structures of sulfides and other chalcogenides. In Sulfide Mineralogy and Geochemistry; Vaughan, D.J., Ed.; Mineralogical Society of America Reviews in Mineralogy and Geochemistry: Sully, VA, USA, 2006; Volume 61, pp. 7-125. [CrossRef]

67. Britvin, S.N.; Bogdanova, A.N.; Boldyreva, M.M.; Aksenova, G.Y. Rudashevskyite, the Fe-dominant analogue of sphalerite, a new mineral: Description and crystal structure. Am. Miner. 2008, 93, 902-909. [CrossRef]

68. Vaughan, D.J. (Ed.) Sulfide Mineralogy and Geochemistry; Mineralogical Society of America Reviews in Mineralogy and Geochemistry: Sully, VA, USA, 2006; Volume 61, p. 714.

69. Toulmin, P.; Barton, P.B.; Wiggins, L.B. Commentary on the sphaleritegeobarometer. Am. Miner. 1991, 76, 1038-1051. [CrossRef]

70. Kaneko, S.; Aoki, H.; Kawahara, Y.; Imoto, F.; Matsumoto, K. Solid solutions and phase transformations in the system ZnS-CdS under hydrothermal conditions. J. Electrochem. Soc. 1984, 131, 1445-1446. [CrossRef]

71. Tomashyk, V.; Feychuk, P.; Scherbak, L. Ternary Alloys Based on II-Vi Semiconductor Compounds, 1st ed.; CRC Press: Boca Raton, FL, USA, 2013; p. 560.

72. Lin, Y.; El Goresy, A. A comparative study of opaque phases in Qingzhen (EH3) and MacAlpine Hills 88136 (EL3): Representatives of EH and EL parent bodies. Meteorit. Planet. Sci. 2002, 37, 577-599. [CrossRef]

73. Sokol, E.V.; Deviatiiarova, A.S.; Kokh, S.N.; Reverdatto, V.V.; Artemyev, D.A.; Kolobov, V.Y. Sulfide mineralization hosted by spurrite-mervinite marbles (Kochumdek River, East Siberia). Dokl. Earth Sci. 2019, 489, 1326-1329. [CrossRef]

74. Chaplygin, I.V.; Mozgova, N.N.; Mokhov, A.V.; Koporulina, E.V.; Bernhardt, H.J.; Bryzgalov, I.A. Minerals of the system ZnS-CdS from fumaroles of the Kudriavy volcano, Iturup Island, Kuriles, Russia. Can. Miner. 2007, 45, 709-722. [CrossRef]

75. Chaplygin, I.V. Ore Mineralization of High-Temperature Fumaroles of Kudriavy Volcano (Iturup Island, Kurile Islands). Ph.D. Thesis, IGEM RAS, Moscow, Russia, 2009. (In Russian)

76. Vaughan, D.; Craig, J. Mineral Chemistry of Metal Sulfides; Cambridge University Press: Cambridge, UK, 1986.

77. Fedorov, V.A.; Ganshin, V.A.; Korkishko, Y.N. Solid-state phase diagram of the zinc sulfide-cadmium sulfide system. Mater. Res. Bull. 1993, 28,59-66. [CrossRef]

78. Vasil'ev, V.I. New data on the composition of metacinnabar and Hg-sphalerite with an isomorphous Cd admixture. Russ. Geol. Geophys. 1991, 52, 701-708. [CrossRef]

79. Grammatikopoulos, T.; Valeyev, O.; Roth, T. Compositional variation in Hg-bearing sphalerite from the polymetallic Eskay Creek deposit, British Columbia, Canada. Chem. Erde 2006, 66, 307-314. [CrossRef]

80. Orberger, B.; Pasava, J.; Gallien, J.-P.; Daudin, L.; Trocellier, P. Se, As, Mo, Ag, Cd, In, Sb, Pt, Au, Tl, Re traces in biogenic and abiogenic sulfides from Black Shales (Selwyn Basin, Yukon territories, Canada): A nuclear microprobe study. Nucl. Instrum. Methods Phys. Res. 2003, 210, 441-448. [CrossRef]

81. Pirri, I.V. On the occurrence of selenium in sulfides of the ore deposit of Baccu Locci (Gerrei, SE Sardinia). Neues Jahrb. Mineral. Mon. 2002, 2002, 207-224. [CrossRef]

82. Ueno, T.; Scott, S.D. Solubility of gallium in sphalerite and würtzite at $800^{\circ} \mathrm{C}$ and $900{ }^{\circ} \mathrm{C}$. Can. Miner. 1991, 29, 143-148.

83. Johan, Z. Indium and germanium in the structure of sphalerite: An example of coupled substitution with copper. Miner. Petrol. 1988, 39, 211-229. [CrossRef]

84. Ciobanu, C.L.; Cook, N.J.; Pring, A.; Damian, G.; Capraru, N. Another look at nagyágite from the type locality, Săcărîmb, Romania: Replacement, chemical variation and petrogenetic implications. Miner. Petrol. 2008, 93, 273-307. [CrossRef]

85. Ye, L.; Cook, N.J.; Liu, T.; Ciobanu, C.L.; Gao, W.; Yang, Y. The Niujiaotang Cd-rich zinc deposit, Duyun, Guizhou province, southwest China: Ore genesis and mechanisms of cadmium concentration. Miner. Depos. 2012, 47, 683-700. [CrossRef]

86. Sokol, E.V.; Volkova, N.I. Combustion metamorphic events resulting from natural coal fires. In GSA Reviews in Engineering Geology XVIII: Geology of Coal Fires: Case Studies from around the World; Stracher, G.B., Ed.; The Geological Society of America: Boulder, CO, USA, 2007; pp. 97-115.

87. Grapes, R.; Korzhova, S.; Sokol, E.; Seryotkin, Y. Paragenesis of unusual Fe-cordierite (sekaninaite)-bearing paralava and clinker from the Kuznetsk coal basin, Siberia, Russia. Contrib. Miner. Petrol. 2011, 162, 253-273. [CrossRef]

88. Sokol, E.; Sharygin, V.; Kalugin, V.; Volkova, N.; Nigmatulina, E. Fayalite and kirschsteinite solid solutions in melts from burned spoil-heaps, South Urals, Russia. Eur. J. Miner. 2002, 14, 795-807. [CrossRef] 
89. Marks, M.A.W.; Wenzel, T.; Whitehouse, M.J.; Loose, M.; Zack, T.; Barth, M.; Worgard, L.; Krasz, V.; Eby, G.N.; Stosnach, H.; et al. The volatile inventory $(\mathrm{F}, \mathrm{Cl}, \mathrm{Br}, \mathrm{S}, \mathrm{C})$ of magmatic apatite: An integrated analytical approach. Chem. Geol. 2012, 291, 241-255. [CrossRef]

90. Parat, F.; Dungan, M.A.; Streck, M.J. Anhydrite, pyrrhotite, and sulfur-rich apatite: Tracing the sulfur evolution of an Oligocene andesite (Eagle Mountain, CO, USA). Lithos 2002, 64, 63-75. [CrossRef]

91. Zateeva, S.N.; Sokol, E.V.; Sharygin, V.V. Specificity of pyrometamorphic minerals of the ellestadite group. Geol. Ore Depos. 2007, 49, 792-805. [CrossRef]

92. Kokh, S.N.; Sokol, E.V.; Sharygin, V.V. Ellestadite-group minerals in combustion metamorphic rocks. In Coal and Peat Fires: A Global Perspective; Stracher, G.B., Prakash, A., Sokol, E.V., Eds.; Elsevier: Amsterdam, The Netherlands, 2015; Volume 3, Chapter 20; pp. 543-562.

93. Khoury, H.N. High- and low-temperature mineral phases from the pyrometamorphic rocks, Jordan. Arab. J. Geosci. 2020, 13, 734. [CrossRef]

94. Galuskin, E.V.; Gfeller, F.; Galuskina, I.O.; Pakhomova, A.; Armbruster, T.; Vapnik, Y.; Wlodyka, R.; Dzierżanowski, P.; Murashko, M. New minerals with a modular structure derived from hatrurite from the pyrometamorphic Hatrurim Complex. Part II. Zadovite, $\mathrm{BaCa}_{6}\left[\left(\mathrm{SiO}_{4}\right)\left(\mathrm{PO}_{4}\right)\right]\left(\mathrm{PO}_{4}\right)_{2} \mathrm{~F}$ and aradite, $\mathrm{BaCa}_{6}\left[\left(\mathrm{SiO}_{4}\right)\left(\mathrm{VO}_{4}\right)\right]\left(\mathrm{VO}_{4}\right)_{2} \mathrm{~F}$, from paralavas of the Hatrurim Basin, Negev Desert, Israel. Miner. Mag. 2015, 79, 1073-1087. [CrossRef]

95. Sharygin, V.V.; Sokol, E.V.; Vapnik, Y. Minerals of the pseudobinary perovskite-brownmillerite series from combustion metamorphic larnite rocks of the Hatrurim Formation (Israel). Russ. Geol. Geophys. 2008, 49, 709-726. [CrossRef]

96. Danilovsky, V.A.; Sokol, E.V.; Karmanov, N.S.; Kokh, S.N.; Devyatiyarova, A.S.; Sharygin, V.V.; Nigmatulina, E.N. Ca-Fe high-temperature oxysulfide: First finding in nature. Nat. Sci. 2018, 2, 32-37. (In Russian)

97. Rosenqvist, T. Phase equilibria in the pyrometallurgy of sulfide ores. Metall. Mater. Trans. B 1978, 9, 337-351. [CrossRef]

98. Jha, A.; Grieveson, P. Carbothermic reduction of pyrrhotite in the presence of lime for the production of metallic iron. II: Kinetics and mechanism of reduction. Scand. J. Metall. 1992, 21, 50-62.

99. Jha, A.; Tang, S.; Chrysanthou, A. Phase equilibria in the metal-sulfur-oxygen system and selective reduction of metal oxides and sulfides: Part I. The carbothermic reduction and calcination of complex mineral sulfides. Metall. Mater. Trans. B 1996, 27, 829-840. [CrossRef]

100. Selivanov, E.N.; Chumarev, V.M.; Gulyaeva, R.I.; Mar'evich, V.P.; Vershinin, A.D.; Pankratov, A.A.; Korepanova, E.S. Composition, structure, and thermal expansion of $\mathrm{Ca}_{3} \mathrm{Fe}_{4} \mathrm{~S}_{3} \mathrm{O}_{6}$ and CaFeSO. Inorg. Mater. 2004, 40, 845-850. (In Russian) [CrossRef]

101. Gulyaeva, R.I.; Selivanov, E.N.; Mansurova, A.N. Kinetics of the calcium oxysulfides reduction by carbon monoxide. Defect Diffus. Forum 2009, 283, 539-544. [CrossRef]

102. Kopylov, N.I.; Lata, V.A.; Toguzov, M.Z. Interactions and Phase States in Molten Sulfide Systems; Gylym: Almaty, Kazakhstan, 2001.

103. Polat, I.; Aksu, S.; Altunbas, M.; Bacaks, E. Microstructural, optical and magnetic properties of cobalt-doped zinc oxysulfide thin films. Mater. Chem. Phys. 2011, 130, 800-805. [CrossRef]

104. Cheng, Q.; Wang, D.; Zhou, H. Electrodeposition of $\mathrm{Zn}(\mathrm{O}, \mathrm{S})$ (zinc oxysulfide) thin films: Exploiting its thermodynamic and kinetic processes with incorporation of tartaric acid. J. Energy Chem. 2017, 27, 913-922. [CrossRef]

105. Selivanov, E.; Gulyaeva, R. Chemistry and kinetics (TG/DTA-MS) of metals carbothermical reduction in the FeS- $\mathrm{Ni}_{3} \mathrm{~S}_{2}-\mathrm{CaO}$ system. In Proceedings of the 13th Israeli-Russian Bi-National Workshop “The Optimization of Composition, Structure and Properties of Metals, Oxides, Composites, Nanoand Amorphous Materials", Yekaterinburg, Russia, 13-18 September 2014; pp. 204-220.

106. Schwartz, M.O. Cadmium in zinc deposits: Economic geology of a polluting element. Int. Geol. Rev. 2000, 42, 445-469. [CrossRef]

107. Achternbosch, M.; Bräutigam, K.-R.; Hartlieb, N.; Kupsch, C.; Richers, U.; Stemmermann, P. Heavy metals in cement and concrete resulting from the co-incineration of wastes in cement kilns with regard to the legitimacy of waste utilization. In Forschungszentrum Karlsruhe in der Helmholtz-Ge-Meinschaft Wissenschaftliche Berichte FZKA 6923; Umwelt Bundes Amt.: Dessau-Roßlau, Germany, 2003; 200p. 
108. Deng, S.; Shi, Y.; Liu, Y.; Zhang, C.; Wang, X.; Cao, Q.; Li, S.; Zhang, F. Emission characteristics of Cd, Pb and $\mathrm{Mn}$ from coal combustion: Field study at coal-fired power plants in China. Fuel Process. Technol. 2014, 126, 469-475. [CrossRef]

109. Cui, W.; Meng, Q.; Feng, Q.; Zhou, L.; Cui, Y.; Li, W. Occurrence and release of cadmium, chromium, and lead from stone coal combustion. Int. J. Coal Sci. Technol. 2019, 6, 586-594. [CrossRef]

110. Vapnik, Y.; Galuskina, I.; Palchik, V.; Sokol, E.V.; Galuskin, Y.; Lindsley-Griffin, N.; Stracher, G.B. Stone-tool workshops of the Hatrurim Basin, Israel. In Coal Peat Fires: A Glob. Perspect. Case Studies-Coal Fires; Stracher, G.B., Prakash, A., Sokol, E.V., Eds.; Elsevier: Amsterdam, The Netherlands, 2015; Volume 3, pp. 3282-3316. [CrossRef]

111. Becker, W.; Lutz, H.D. Phase studies in the systems CoS-MnS, CoS-ZnS, and CoS-CdS. Mater. Res. Bull. 1978, 13, 907-911. [CrossRef]

112. Wu, P.; Kershaw, R.; Dwight, K.; Wold, A. Growth and characterization of nickel-doped ZnS single crystals. Mater. Res. Bull. 1989, 24, 49-53. [CrossRef]

113. Vapnik, Y.; Sokol, E.; Murashko, M.; Sharygin, V. The enigma of Hatrurim. Mineral. Alm. 2006, 10, 69-77.

114. Sharygin, V.V.; Vapnik, Y. Ferrites and silicoferrites in magnetite-hematite nodule, Hatrurim Basin, Israel. In Proceedings of the Conference Mineralogical Museum 2019. Mineralogy Yesterday, Today, Tomorrow, Saint Petersburg, Russia, 17-19 September 2019; pp. 190-192.

115. Britvin, S.N.; Murashko, M.N.; Vapnik, E.; Polekhovsky, Y.S.; Krivovichev, S.V. Barringerite $\mathrm{Fe}_{2} \mathrm{P}$ from pyrometamorphic rocks of the Hatrurim Formation, Israel. Geol. Ore Depos. 2017, 59, 619-625. [CrossRef]

116. Britvin, S.N.; Murashko, M.N.; Vapnik, Y.; Polekhovsky, Y.S.; Krivovichev, S.V.; Vereshchagin, O.S.; Vlasenko, N.S.; Shilovskikh, V.V.; Zaitsev, A.N. Zuktamrurite, $\mathrm{FeP}_{2}$, a new mineral, the phosphide analogue of löllingite, FeAs 2 . Phys. Chem. Miner. 2019, 46, 361-369. [CrossRef]

117. Britvin, S.N.; Murashko, M.N.; Krzhizhanovskaya, M.G.; Vereshchagin, O.S.; Vapnik, Y.; Shilovskikh, V.V.; Lozhkin, M.S. Nazarovite, IMA 2019-013. CNMNC Newsletter No. 50. Miner. Mag. 2019, 83. [CrossRef]

118. Britvin, S.N.; Vapnik, Y.; Polekhovsky, Y.S.; Krivovichev, S.V.; Krzhizhanovskaya, M.G.; Gorelova, L.A.; Vereshchagin, O.S.; Shilovskikh, V.V.; Zaitsev, A.N. Murashkoite, FeP, a new terrestrial phosphide from pyrometamorphic rocks of the Hatrurim Formation, South Levant. Miner. Petrol. 2019, 113, 237-248. [CrossRef]

119. Morozova, N.K.; Malov, M.M.; Veselkova, M.M.; Kurbatov, B.A. Oxygen-phase-state changes in ZnS single crystals annealed in vapors of the constituents. Sov. Phys. J. 1975, 18, 672-676. [CrossRef]

120. Morozova, N.K.; Karetnikov, I.A.; Golub, K.V.; Danilevich, N.D.; Lisitsyn, V.M.; Oleshko, V.I. The effect of oxygen on the ZnS electronic energy-band structure. Semiconductors 2005, 39, 485-492. [CrossRef]

121. Lepetit, P.; Bente, K.; Doering, T.; Luckhaus, S. Crystal chemistry of Fe-containing sphalerites. Phys. Chem. Miner. 2003, 30, 185-191. [CrossRef]

122. Wang, Z.; Wang, F.; Wang, L.; Jia, Y.; Sun, Q. First-principles study of negative thermal expansion in zinc oxide. J. Appl. Phys. 2013, 114, 063508. [CrossRef]

123. Muñoz-Aguirre, N.; Martínez-Pérez, L.; Muñoz-Aguirre, S.; Flores-Herrera, L.A.; Vergara Hernández, E.; Zelaya-Angel, O. Luminescent properties of (004) highly oriented cubic zinc blende $\mathrm{ZnO}$ thin films. Materials 2019, 12, 3314. [CrossRef] [PubMed]

124. He, Y.; Wang, L.; Zhang, L.; Li, M.; Shang, X.; Fang, Y.; Chen, C. Solubility limits and phase structures in epitaxial ZnOS alloy films grown by pulsed laser deposition. J. Alloy Compd. 2012, 534, 81-85. [CrossRef]

125. Kramchenkov, A.B.; Kurbatov, D.I.; Zaharets, M.I.; Opanasyuk, A.S. Investigation of oxygen content in zinc sulfide thin films using RBS. J. Surf. Investig. X-ray Synchrotron Neutron Tech. 2010, 3, 45-47. (In Russian)

(C) 2020 by the authors. Licensee MDPI, Basel, Switzerland. This article is an open access article distributed under the terms and conditions of the Creative Commons Attribution (CC BY) license (http://creativecommons.org/licenses/by/4.0/). 